University of Redlands

\title{
Fire Emergency Management System for Joshua Tree National Park
}

\author{
A Major Individual Project submitted in partial satisfaction of the requirements \\ for the degree of Master of Science in Geographic Information Systems \\ by \\ Mohammed AlSharif \\ Douglas Flewelling, Ph.D., Committee Chair \\ Fang Ren, Ph.D.
}

December 2010 
Fire Emergency Management System for Joshua Tree National Park

Copyright $@ 2010$

by

Mohammed AlSharif 
The report of Mohammed AlSharif is approved.

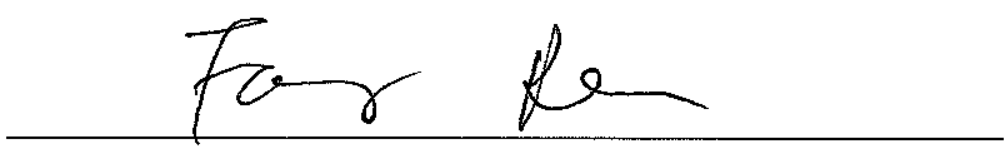

Fang Ren, Ph.D.

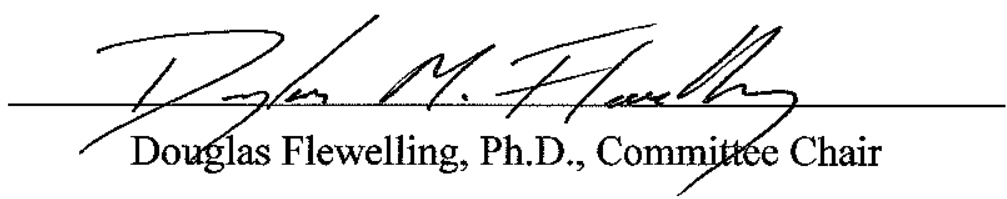

December 2010 



\section{Acknowledgements}

I would like to express my gratitude and thanks to Allah for putting all of the following people in my way so, I got all the support and assistance.

I would like to thank His Excellency the Minister of Interior-Kingdom of Bahrain, Sheikh Rashid bin Abdulla Al-Khalifa, Major-General Team Abdullatif Al-Zayani-Chief of public security prior, Major-General Basim Al-Hamer-President of Customs, Lt. Col Waleed Al-Hamden - Director of Works Directorate for their continued support in the development and upgrading of GIS in Ministry of Interior-Kingdom of Bahrain. I would like to thank them for the opportunity and guidance that enabled me to study Masters of Science in Geographic Information System at University of Redlands- USA.

I would to thank Jack Dangermond - founder and president of ESRI. To all ESRI and ESRINeA staff who help me to complete this project successfully.

I would also like to thank the University of Redlands and the professors for the opportunity, education, and guidance that enabled me to complete this project. In particular, I give my gratitude to my advisor, Douglas Flewelling, Ph.D., for his guidance and input. I would like to give many thanks for Joshua Tree National Park GIS Specialist Sean Murphy, whose timely support and enthusiasm with the project allowed me to ensure successful completion.

I would like to additionally thank Debra Riley, Program Coordinator, for her words of encouragement and for keeping everyone organized. I also want to thank Ruben Ortiz, and Steve Paplanus for their excellent IT support. I am also grateful to Lynn Flewelling for her providing detailed edits of my report. I want to give a credit to Nathan Strout, Bjorn, and all the Redlands Institute staff for their assistance during critical stages of this project.

To my parents, thank you for encouragement, prayer, and tremendous support. To Sara, thank you a lot for your big support, and encourage me to be patient. To my family, thank you for all your support. To my sister Noor, thank you for your support, and your delicious food. To Michelle and Renee, I would like to thank you a lot. To International Friendship connection (Especially for Nadean and Wayne), thank you for introducing me to American traditions and helping me a lot here in Redlands.

To Cohort 17 - I cannot imagine a better group of people to have shared this experience with. The MS GIS faculty and my fellow cohort members have made this a very rewarding, fun, and intense year of learning. I would like to extend my deep appreciation to all my friends for supporting me all the times. Lastly, I would like to thank all the people (Especially Aseel and Ali) who have assisted me in spending this meaningful year in MS GIS program.

At the end, I would like to say that: I am a GIS Specialist, I can be an Analyst or an Artist, I can be Engineer, or whatever I want. I am famous, I am a GIS Specialist. 



\author{
Abstract \\ Fire Emergency Management System for Joshua Tree National Park \\ by \\ Mohammed AlSharif
}

Geographic information system (GIS) technology is a valuable tool when it comes to assessing and managing the emergency response to wild fire. Joshua Tree National Park (JOTR) recognized a need to deliver a GIS-based system to assist them with more rapid response to wildfire incidents and to prioritize creation of firebreaks, and the benefits of the technology to describe an automated solution that responds to fire when identified. This document outlines the procedures for using GIS to provide a solution for this problem in JOTR. The solution involved producing Python scripting tools to prepare the data for subsequent input to geoprocessing tools and designing a web-based application that would help the fire dispatch center understand where a response is needed. The web application enabled the user to identify the fire incident location using different types of searches, identify the shortest path to the fire from the fire station, and identify the best places for the firebreak. 



\section{Table of Contents}

Chapter 1 - Introduction ................................................................................................ 1

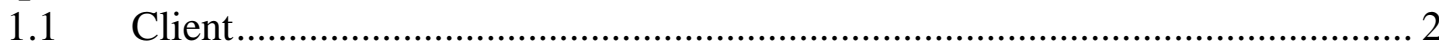

$1.2 \quad$ Problem Statement ........................................................................... 2

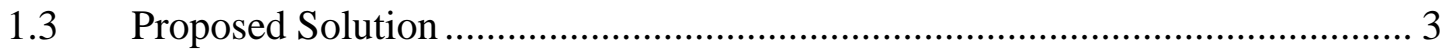

1.3.1 Goals and Objectives ........................................................................... 3

1.3.2 Scope

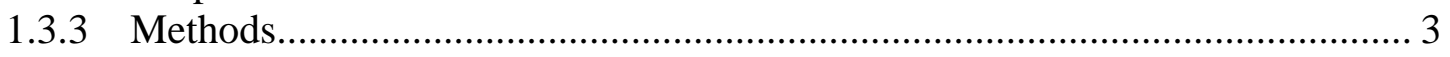

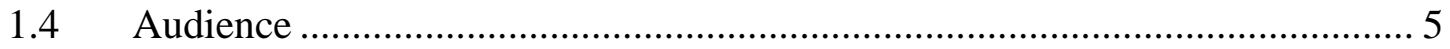

1.5 Overview of the Rest of this Report ......................................................... 5

Chapter 2 - Background and Literature Review .............................................................. 7

2.1 GIS Technology and Fire Emergency Management....................................... 7

2.2 Cost Surface Analysis ........................................................................ 9

$2.3 \quad$ Suitability Analysis ............................................................................. 9

2.4 Web-Based GIS Application in Fire Emergency Projects ................................. 9

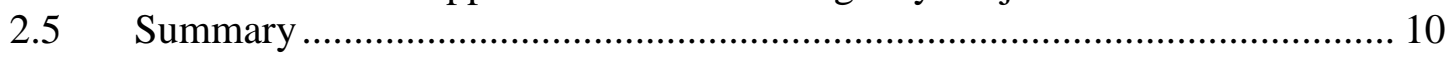

Chapter 3 - Systems Analysis and Design...................................................................... 11

$3.1 \quad$ Problem Statement ............................................................................. 11

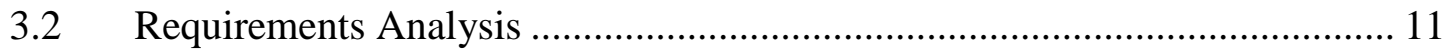

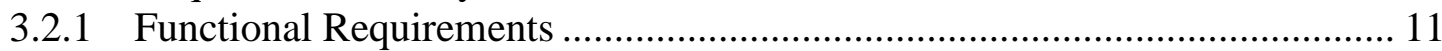

3.2.2 Non-functional Requirements ................................................................. 13

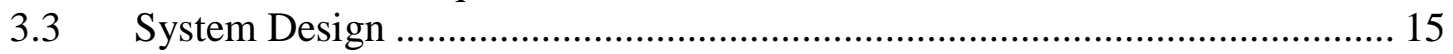

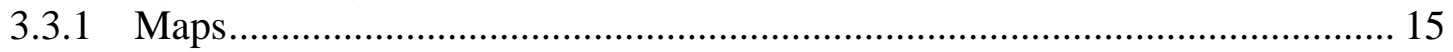

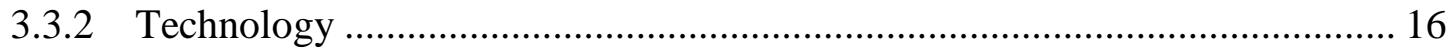

3.3.3 System Architecture ............................................................................... 17

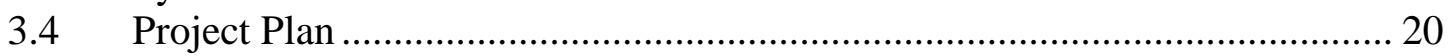

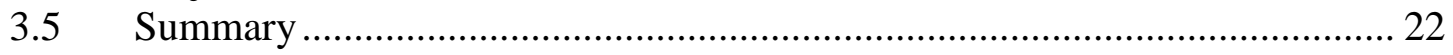

Chapter 4 - Database Design......................................................................................... 23

4.1 Conceptual Data Model ........................................................................ 23

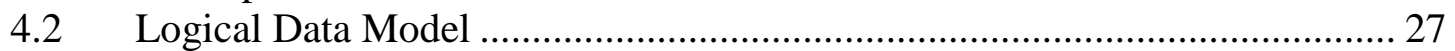

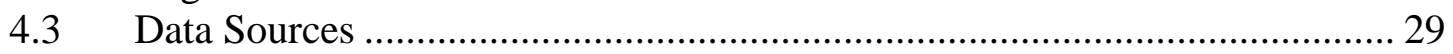

4.4 Data Scrubbing and Loading …………………....................................... 32

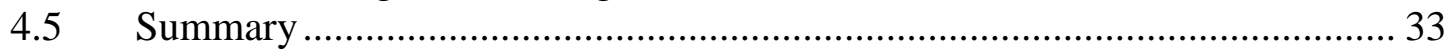

Chapter 5 - Implementation.................................................................................................. 35

$5.1 \quad$ Cost Surface Analysis Scripting Tool......................................................... 35

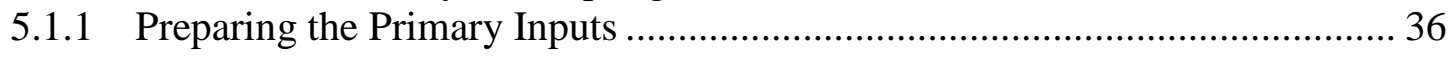

5.1.2 Run the Cost Distance Tool ........................................................................... 42

5.2 Firebreak Preparing Data Scripting Tool ..................................................... 43

5.3 Other Python Scripting Tools ........................................................................ 47

5.3.1 Least Cost Path Scripting Tool …………………….................................... 47

5.3.2 Firebreak Priority Analysis Scripting Tool...…………………………….... 48

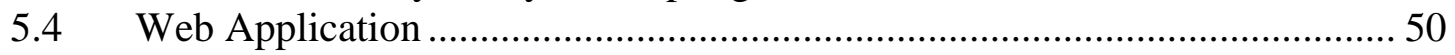

5.4.1 Publishing a Map Service Using ArcCatalog …………………………….... 50 
5.4.2 Developing Geoprocessing Services and a Geometry Service ....................... 54

5.4.3 Developing a Web Application Using Flex API............................................ 56

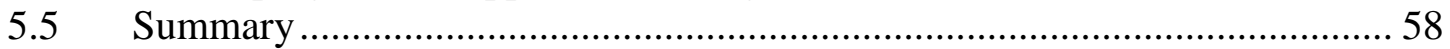

Chapter 6 - Results and Analysis....................................................................................... 59

6.1 GIS Tools Installation and Results ........................................................59

6.1.1 Cost Surface Analysis Tool Results.......................................................... 59

6.1.2 Firebreak Preparing Data Tool Results............................................................. 64

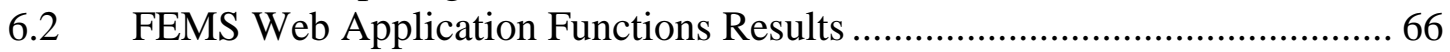

6.2.1 Web Application Functions Results............................................................... 66

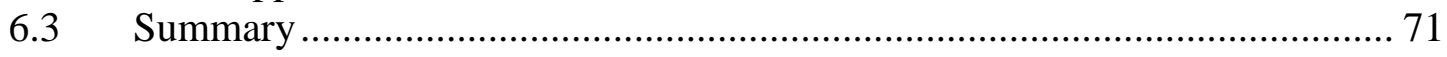

Chapter 7 - Conclusions and Future Work .............................................................. 73

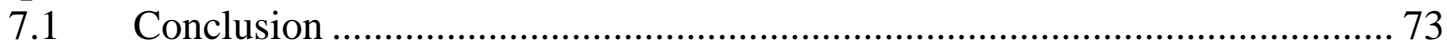

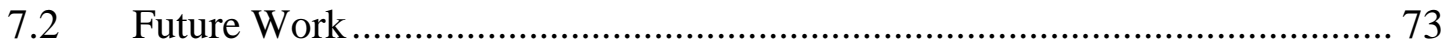

7.2.1 Enhancing the Firebreak Priority Analysis tool............................................. 73

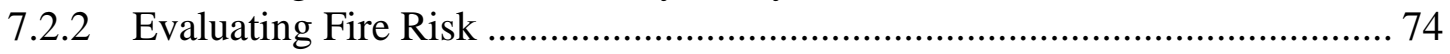

7.2.3 Analyzing Fire Spread …………………………............................... 74

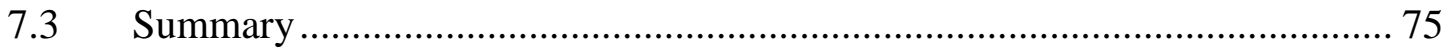

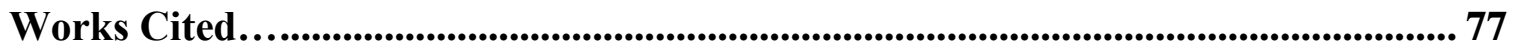

Appendix A. Python Scripting Tools................................................................................ 79

Appendix B. Web GIS Application Code ..................................................................... 87 


\section{Table of Figures}

Figure 1-1

Figure 1-2

Figure 1-3

Figure 3-1

Figure 3-2

Figure 3-3

Figure 3-4

Figure 3-5

Figure 4-1

Figure 4-2

Figure 4-3

Figure 4-4

Figure 4-5

Figure 4-6

Figure 5-1

Figure 5-2

Figure 5-3

Figure 5-4

Figure 5-5

Figure 5-6

Figure 5-7

Figure 5-8

Figure 5-9

Figure 5-10

Figure 5-11

Figure 5-12

Figure 5-13

Figure 5-14

Figure 5-15

Figure 5-16

Figure 5-17

Figure 5-18

Figure 5-19

Figure 5-20

Figure 5-21

Figure 5-22

Figure 5-23

Figure 5-24

Figure 5-25

Figure 5-26

Figure 5-27

Figure 5-28

Figure 6-1
Lost Horse Mine Fire at JOTR. 1

Joshua Tree National Park General Layout ........................................ 2

Spiral Process Model ............................................................... 4

FEMS Base Map ........................................................................ 15

Scale Range in FEMS Base Map ..................................................... 16

FEMS Web Application Design ................................................ 17

System Architecture ..................................................................... 19

Preliminary Project Schedule ................................................... 21

System Conceptual Data Model.........................................................2 24

Protected Area Conceptual Data Model ...........................................25

Response to Fire Conceptual Data Model .......................................26

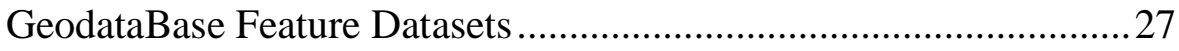

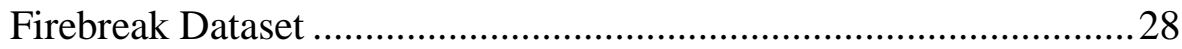

BaseMap Dataset ......................................................................... 28

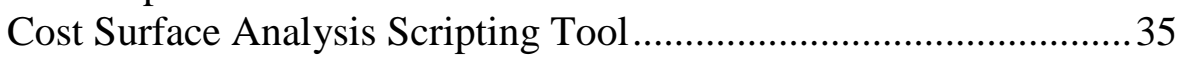

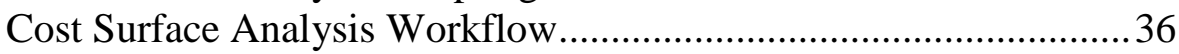

Roads and Trails Feature Classes Preparing Steps .......................... 36

Final Roads Feature Class.......................................................... 37

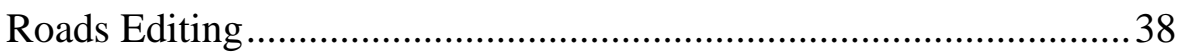

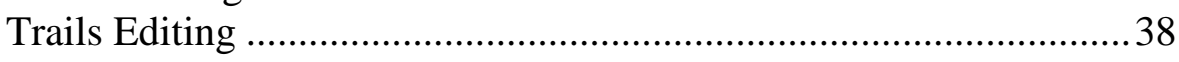

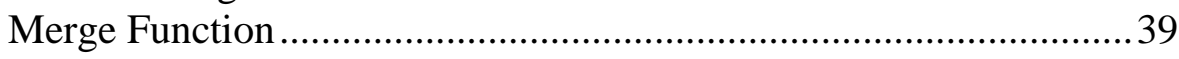

The Output of FeatureToRaster Function ...................................... 39

The Output of Map Algebra.............................................................40

Overall Steps for Creating the Travel Cost Surface.........................40

Slope Values ........................................................................... 41

Firebreak Preparing Data Script Tool ............................................ 43

Firebreak Preparing Data Tool Process ......................................... 44

Overall Steps of Scripting Tool ............................................... 45

Concat Python Function Output......................................................46

Least Cost Path Scripting tool...................................................... 47

Overall Function in Least Cost Path Tool.......................................... 48

Firebreak Priority Analysis Scripting Tool................................... 49

Overall Functions for Firebreak Priority Tool .................................49

FEMS BaseMap Document Overview............................................51

Map Document Editing Option.....................................................52

FEMS BaseMap Web Service .....................................................53

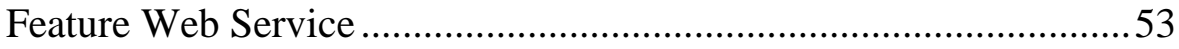

Geoprocessing Services .............................................................54

Selecting the Scripting Toolbox and the Execution Type ................55

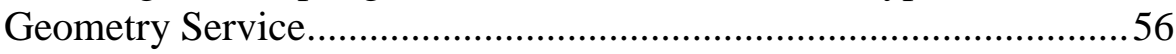

Web Application Workflow Process .............................................5 57

Web Application Different Types of Search ...................................58

Adding the FEMSDesktop Toolbox ............................................59 


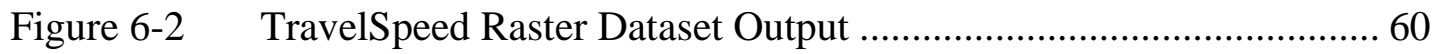

Figure 6-3 Travel Cost Raster Dataset Output .................................................... 61

Figure 6-4 Cost Distance and Backlink Raster Datasets for NPS Fire Station ... 62

Figure 6-5 Cost Distance and Backlink Raster Datasets for BLM Fire Station.. 63

Figure 6-6 Firebreak Preparing Data Tool Primary Inputs .................................. 64

Figure 6-7 Protected Areas Features Class …………………………………........6 65

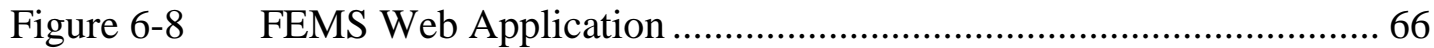

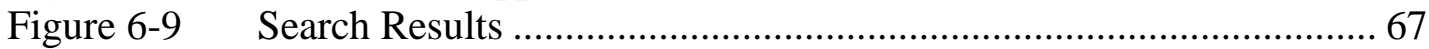

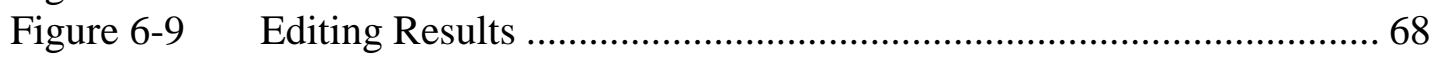

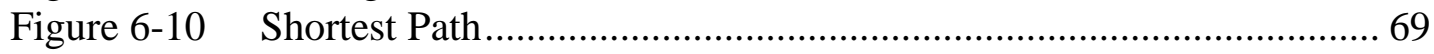

Figure 6-11 Firebreak Priority Analysis Tool Output............................................ 69

Figure 6-12 Using Drawing Tools .................................................................. 70

Figure 6-13 Using Slider Window To Show Wilderness Areas ............................ 70

Figure 7-1 Analyze Fire Spread …………………..................................... 74 


\section{List of Tables}

Table 3-1 Functional Requirements for the Cost Surface Analysis Scripting Tool ...... 12

Table 3-2 Functional Requirements for the Firebreak Factors Scripting Tool ............. 12

Table 3-3 Functional Requirements for the Web Application .................................. 12

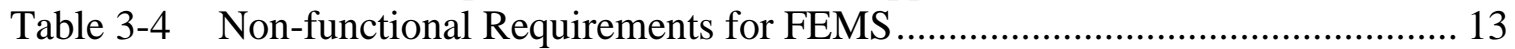

Table 4-1 Feature Class Attributes Data Types....................................................... 29

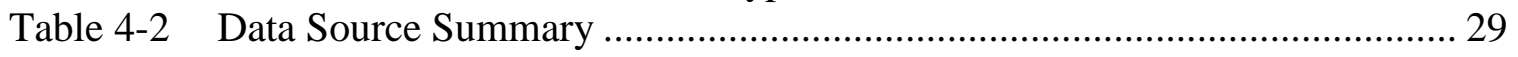

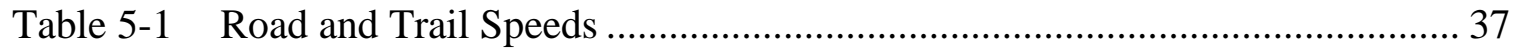

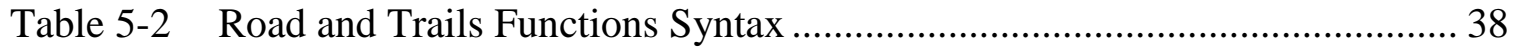

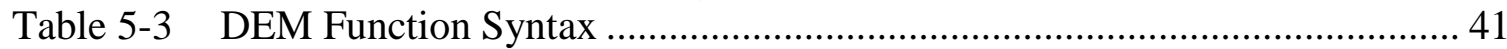

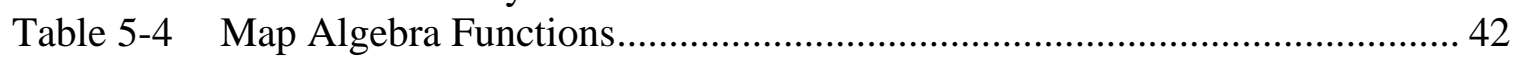





\section{List of Acronyms and Definitions}

$\begin{array}{ll}\text { GIS } & \text { Geographic information system } \\ \text { PLSS } & \text { Public Land Survey System } \\ \text { USGS } & \text { United States Geological Survey } \\ \text { JOTR } & \text { Joshua Tree National Park } \\ \text { NPS } & \text { National Park Service } \\ \text { API } & \text { Application programming interface } \\ \text { CEM } & \text { Comprehensive emergency management } \\ \text { FEM } & \text { Fire emergency management } \\ \text { CSA } & \text { Cost surface analysis } \\ \text { CAD } & \text { Computer aided dispatch } \\ \text { GSS } & \text { Geographic Security System } \\ \text { FEMS } & \text { Fire Emergency Management System } \\ \text { DEM } & \text { Digital elevation model } \\ \text { Esri } & \text { Environmental Systems Research Institute, Inc. } \\ \text { UML } & \text { Unified Model Language } \\ \text { SDE } & \text { Spatial Database Engine } \\ \text { MPH } & \text { Miles per hour }\end{array}$





\section{Chapter 1 - Introduction}

Joshua Tree National Park (JOTR) is part of the National Park Service, which is administrated by the Department of Interior. The park, located in the southeastern California, covers 789,745 acres of land. JOTR experiences fires that may burn for hours or even days before they are reported to the park's fire stations. In 2009, the Lost Horse Mine Fire burned for three days and left around 350 acres of post-burn land (Figure 1.1)

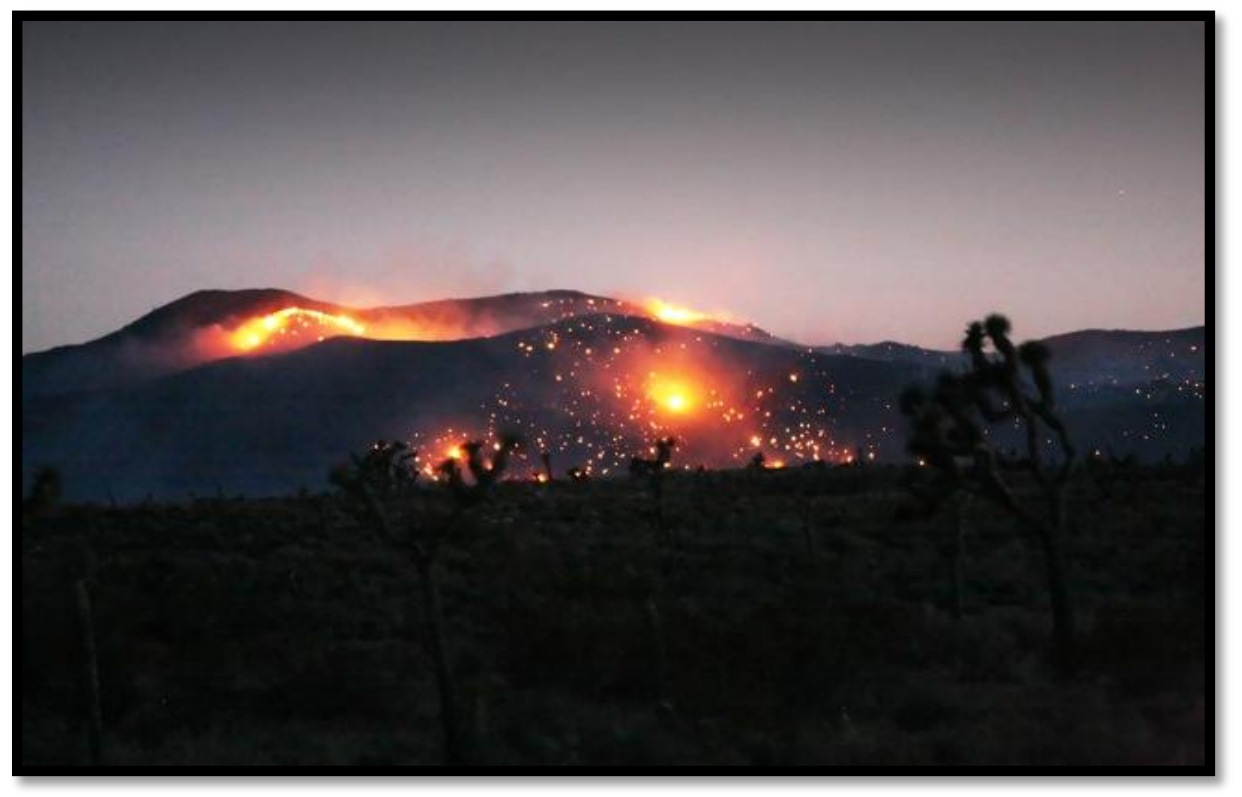

Figure 1-1 Lost Horse Mine Fire at JOTR

The purpose of this project was to use a geographic information system (GIS) and an ArcGIS application-programming interface for Flex (Flex API) to provide assist JOTR with more rapid response to wildfire incidents and to prioritize creation of firebreaks. Moreover, this solution would process the geodatabase to provide an easy way to locate the fire incident. There are three main objectives for this analysis: to identify fire location, to identify the shortest path to the fire from a fire station, and to identify firebreak location.

This chapter is comprised of five sections. The first section discusses the project client. The second section presents the general problem addressed by this project. The third section outlines a summary of the proposed solution, with subsections describing the project goals and objectives, scope, and methodology. The fourth section addresses the primary audience for this project; those who would benefit from the web application solution are described in this paper. Finally, the fifth section presents a brief summary of the topics covered in the remaining chapters of this project report. 


\subsection{Client}

The client for this project was the National Park Service (NPS), in particular the JOTR Fire Dispatch Center. Sean Murphy, a GIS specialist with the NPS, was the primary point of contact for the project, and was supported by John McDuffy who is the Engine Boss for the park's fire trucks. The client needed a GIS solution that could be used to reduce fire damage to the park and help them locate the best places for firebreaks. The client provided primary data, such as roads, trails, boundary, public land survey system (PLSS), United States Geological Survey (USGS) place names, local JOTR place names (called Jeff names), inhabited areas, habitats, and the aerial photo imagery (Figure 1-2). In addition, the client provided periodic support throughout the project.

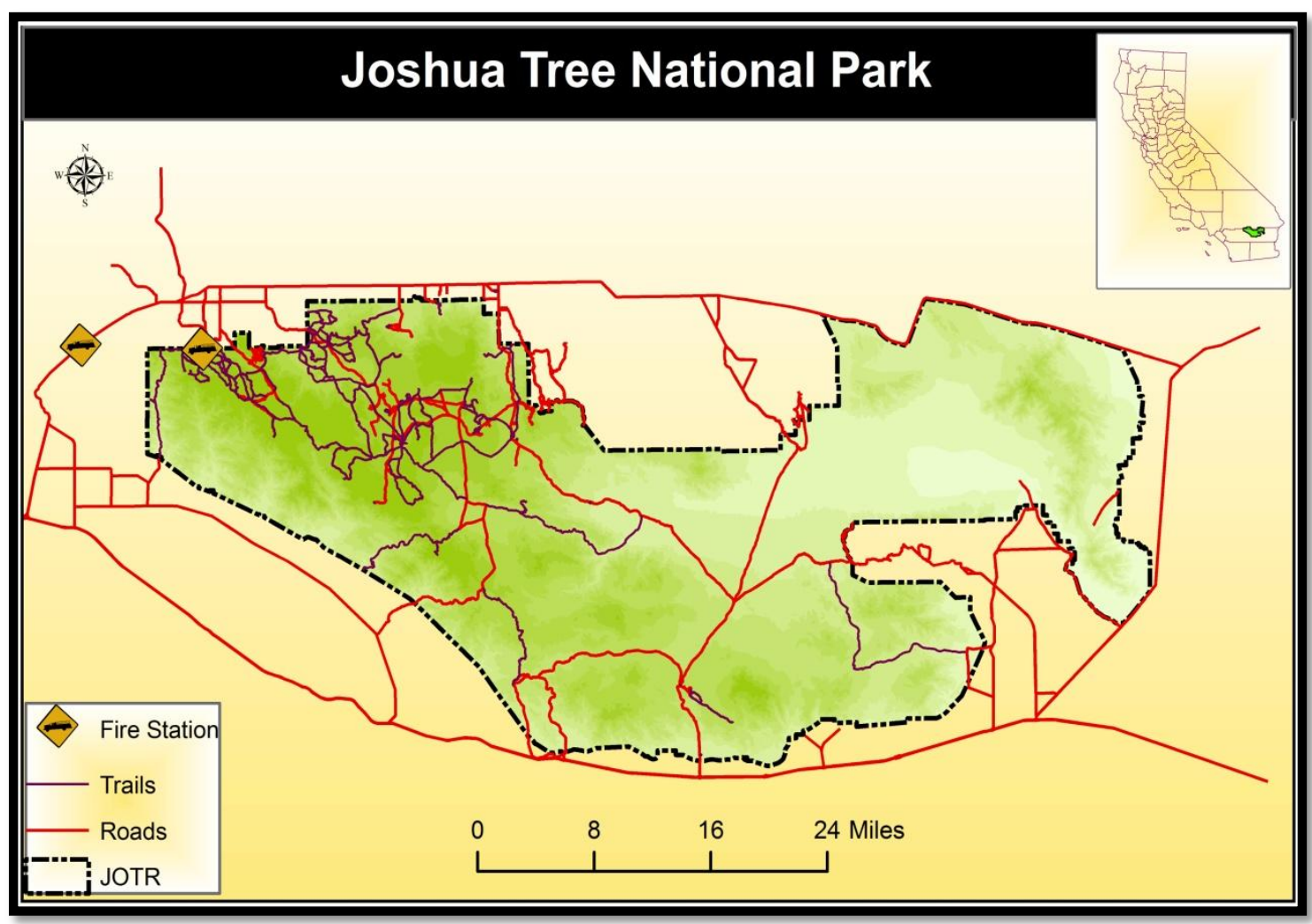

Figure 1-2 Joshua Tree National Park General Layout

\subsection{Problem Statement}

The JOTR was concerned with the threat of fires burning for hours or even days before the park's fire station is informed about them. Since there is not enough information about any fire incident, it was important to help the fire dispatch center assist JOTR with more rapid response to wildfire incidents. In addition, they do not know the shortest path from fire station to the fire incident, and they do not know the best place for firebreaks. A GIS solution was needed to help them identify the place where the fire incidents occur, to find the least cost path to the fire incident, and to find the best place to build firebreaks. 


\subsection{Proposed Solution}

This section discusses the general approach to solving the problem statement described in Section 1.2. It provides details on the goals and objectives of the project. The project scope and methodology are further outlined based on these objectives.

\subsubsection{Goals and Objectives}

The goal of this project was to help the JOTR fire station assess fire incident by developing a web-based application to assist them with rapid response to fire incidents. An additional objective of this project was to reduce the response time for fire emergencies. The specific objectives were:

- Identify the fire incident location using different search types.

- Identify the least cost path from fire station to the incident.

- Identify the best place for firebreaks.

By meeting the goals and objectives, fire emergency management system (FEMS) will allow the JOTR to conduct accurate analyses to help the fire dispatch center to reduce the response time to fire and preserve the park from the fire threat.

\subsubsection{Scope}

The specific scope of this project included: developing a web-based application solution to use for querying a GIS database for a specific place reference and showing where the fire incident is occurring; identifying the quickest path and displaying the best location for firebreaks; presenting the FEMS at the JOTR, and providing complete documentation. The solution works with the data provided by the client, like the JOTR roads network and aerial photo imagery.

\subsubsection{Methods}

The project was developed using a spiral development model, which is a lifecycle development system that combines the features of the prototyping model and the waterfall model. In addition, it facilitates a high level of risk analysis, and is more suitable for designing and managing projects when the requirements are not clear from the beginning and not stable. It consists of six sectors: client communication, planning, system analysis and risk analysis, system design, engineering, and evaluation (Boehm, 1988).

Each loop in the spiral model represents a phase, beginning with collecting the data from the client and identifying his objectives and requirements. All the possible risks that will face the development team in the project development are identified and resolved. The actual development of the project was then carried out and the output of the developed product is passed on to the client for comments and suggestions. The client's comments and suggestions were passed through all phases iteratively in order to improve the web-application and meet all of the client's requirements. (Figure 1-3) 


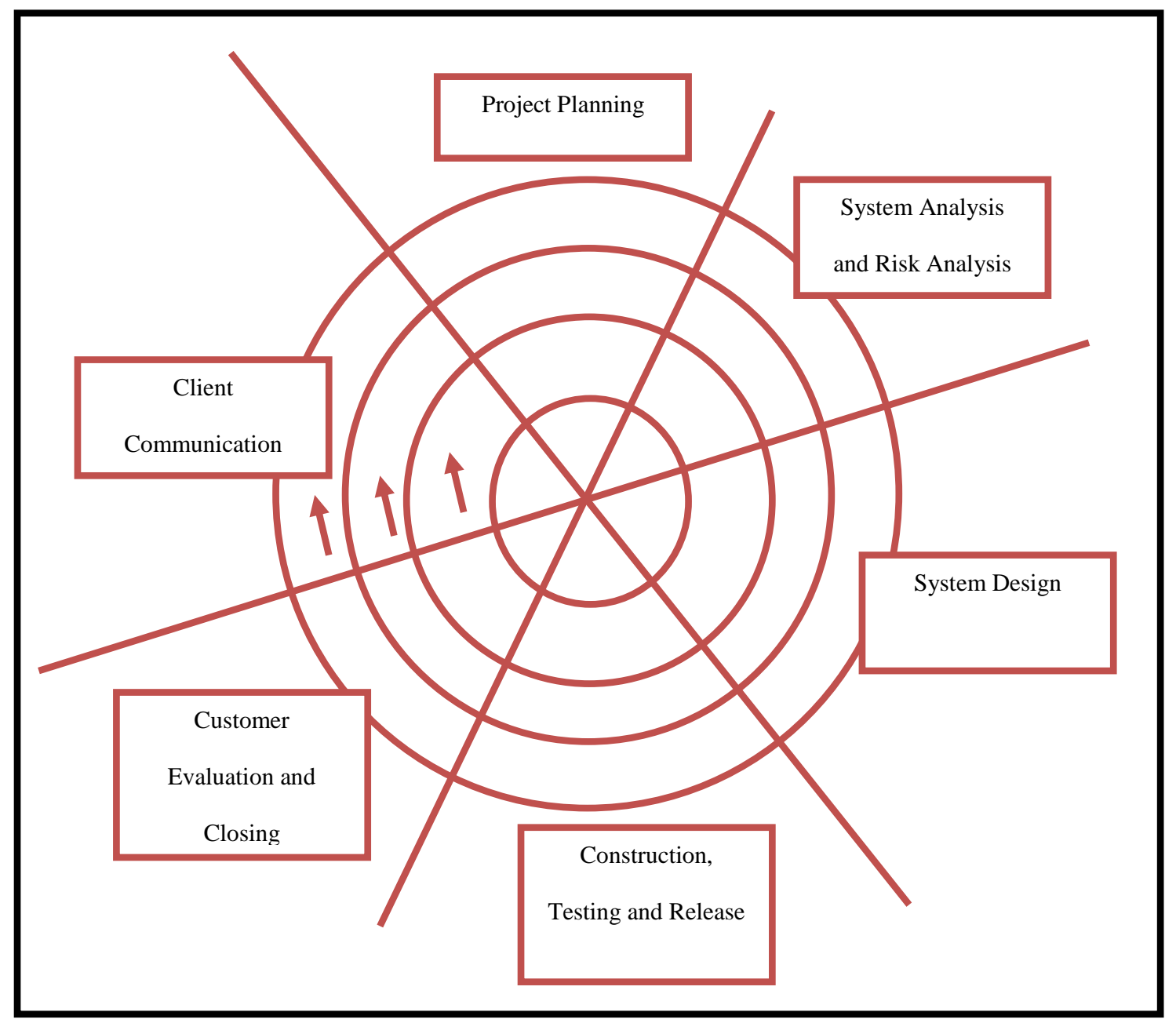

Figure 1-3 Spiral Process Model

This project comprised two major tasks: developing GIS tools and a web-based application. The GIS tools were created using Python scripting programming language and the Environmental Systems Research Institute (ESRI) ArcGIS 9.3.1 package with an ArcInfo license in order to perform a sequence of algorithms. The GIS tools were: Cost Surface Analysis, Firebreak Preparing Data, Least Cost Path, and Firebreak Priority Analysis. The Cost Surface Analysis tool was created to accept a set of inputs and create the Cost Distance and Backlink raster datasets for each fire station. The output of this tool was used as input for the Least Cost Path tool that will run from the web application to create the shortest path from the fire station to the fire incident. In addition, the Firebreak Preparing Data tool was created to accept a set of input and perform a sequence of algorithms to create the Protected Areas feature class. The output of this tool was used as input for the Firebreak Priority Analysis tool to perform a sequence of algorithms and display the output in the web application.

The web-based application employed Flex API as the development platform. To begin, the major layers were added to ArcMap document. The map was saved as mxd file and published to ArcGIS Server 10 as a map service. The Least Cost Path tool and the 
Firebreak Priority Analysis tool were published to the server as a geoprocessing service. In addition, a geometry service was published to the server. The main functions of the web application were: identifying the fire location using different search types, running the Least Cost Path service to identify the least cost path form the fire station to the fire incident, and running the Firebreak Priority tool to identify the best place for firebreaks.

\subsection{Audience}

This report is intended for a general audience with an introductory knowledge of GIS and fire emergency management. The specific intended audience for this report, web application, and the full set of deliverables is the fire dispatch center at JOTR.

\subsection{Overview of the Rest of this Report}

This report is divided into three main categories, including project background, overall approach of the project, and the outcome of the project. The first part continues in Chapter Two with background for the project and a review of pertinent literature from authoritative sources.

The second part is presented in Chapters Three, Four, and Five, which contain the overall approach to the project. Chapter Three identifies the problem statement, describes the requirements analysis, system design, project plan, and provides a summary of the overall system analysis and design of the project. Chapter Four provides the structure of the database design that includes a conceptual and logical data model, source of the data, and methods for obtaining the data. Chapter Five describes the steps taken to complete the project in satisfaction of the stated project goals and objectives.

Finally, Chapter Six addresses the results of the project implementation and Chapter Seven presents the general conclusions of the project and suggestions for future work. 



\section{Chapter 2 - Background and Literature Review}

Intervention to address disasters has evolved through time into a complex policy subsystem, and disaster policy is implemented through a set of functions known as emergency management and response. Modern approaches to emergency management and response involve multidimensional efforts to reduce the vulnerability to hazards; to diminish the impact of disasters; and to prepare for, respond to, and recover from those that occur. Fire is one of those disasters that can threaten inhabited structures, habitats, and vegetation.

A background and literature review for fire emergency management (FEM) was first conducted, from which the solution to the project was developed and implemented. This chapter introduces the role of GIS in fire emergency management through the lens of comprehensive emergency management (CEM) and its five phases: planning, mitigation, preparedness, response, and recovery. In addition, the literature examined here involves the use of cost surface analysis, suitability analysis in fire emergency management, and the web-based GIS applications.

\subsection{GIS Technology and Fire Emergency Management}

GIS is a technology that captures, manages, analyzes, and displays data in ways that reveal information to answer questions and solve problems involving spatial content. In recent years, GIS has become a powerful assessment tool for efforts in FEM because of improved computing speed and large storage capacity. The improvements in technologies have had a significant impact on the way spatial data are acquired, stored, managed, and displayed.

FEM was defined as the discipline and profession of applying science, technology, planning, and management to deal with fires that can injure or kill large numbers of people, do extensive damage to property, and disrupt community life (Drabek and Hoetmer, 1991). In addition, FEM activities can be grouped into five phases that are related by time and function. These are also related to each other and each involves different types of skills (Johnson, 2000). However, FEM activities are planning, mitigation, preparedness, response, and recovery.

Planning involves actions that are taken to analyze and document the possibility of fire disaster and the potential consequences or impact on life, property, and the environment. Mitigation involves actions taken to eliminate or to reduce the degree of long-term risk to human life and property from fire disasters. Preparedness is concerned with actions that are taken in advance to develop operational capabilities and facilitate an effective response to fire disaster. The response phase involves actions that are taken immediately before, during, or directly after a fire occurs, to save lives, minimize damage to property, and enhance the effectiveness of recovery. Finally, the recovery phase is characterized by activity to return life to normal or improved levels of damaged structures (Cova, 1999).

Using GIS in FEM activities is helping many organizations to deal with fire disasters and to reduce their impacts. In the planning phase, when GIS is applied and fire disaster areas are viewed with other map data (roads, inhabited structures, habitats, vegetation, 
etc), emergency management officials can begin to formulate mitigation, preparedness, response, and possible recovery needs (Johnson, 2000). Johnson said GIS facilitates the planning phase by allowing planners to view the appropriate combinations of spatial data through computer-generated maps (Johnson, 2000). In the mitigation phase, utilizing existing databases linked to geographic features in GIS displays the values of fire risk (high, moderate, low) quickly and efficiently. In addition, using GIS can answer many questions that appear in this phase. For example, "where are the fire zones?" "What combination of features vegetation, inhabited structure, habitats, etc constitutes a fire hazard?" A GIS can identify specific slope of categories in combination with certain species of flammable vegetation near homes that could be threatened by wildfires (Johnson, 2000).

In the preparedness and response phase, GIS is utilized primarily to help integrate information from different sources and scales, and to format that information into a single source that can formulate and executed fire emergency response plans (Cova, 1999). In addition, GIS can provide answers to questions such as: "Where should fire stations be located if a five minute response time is expected?" "What is the closest fire station?" "What is the shortest path to fire incident?" A GIS can provide detailed information before the first fire unit arrives. For example, during a commercial building fire, it is possible to identify the closest hydrants, electrical panels, and floor plans of the building while the fire truck is route to the fire (ESRI, 2008). During the recovery phase, GIS can play an important role to help the fire officials to identify the immediate threat to life, property, and the environment. Using GIS during the recovery phase is an initial priority to perform a cursory damage assessment and minimize the time necessary to apply for government relief (Cova, 1999).

Many studies and applications have used GIS technology to enhance decisionmaking in FEM. For example, the Winston-Salem Fire Department uses routing application, which is a GIS application that helps them determine the burning building and the best route to it (Amdahl, 2001). Wilson Police and Fire use GIS to share and combine data to identify common 'hot spots' where a high volume of emergency incidents are being experienced, as well as for developing an overall community safety strategy (ESRI, Mapping the Future of Public Safety). Amdahl explained how to assess risk, map wildfires, and allocate resources to deal with risk through GIS (Amdahl, 2001). For example, using GIS in a fire emergency management can map the area of risk according to building size, type, and risk assessment in a single image that quickly provides analysis for the fire risk. The representatives of fire emergency response referenced a previous project completed by Mei-Po Kwan and Jiyeing Lee, which addressed a similar topic, to implement a GIS system to provide a quick emergency response. They examined the potential of using real time 3D GIS to implement an intelligent GIS-based system to deal with the terrorist attacks (Mei-Po and Lee, 2003). Computer-aided dispatch (CAD) application in geographic information system (GSS) system is an emergency resource dispatching system used for computer-controlled emergency resource dispatching (for example, fire vehicle), resource status, incident reporting, and management information (ESRINEA, 2009). Finally, many of the fire departments such as Wilson Police and Fire department, and Kingdom of Bahrain Fire department used GIS for fire deployment analysis, incident analysis, critical response information, incident management, resources allocation, and fire prevention. 


\subsection{Cost Surface Analysis}

The development of a cost surface analysis (CSA) is generally accomplished by adding two or more raster layers. These layers attempt to account for the effects of elevation, vegetation cover, surface composition, and a wide variety of additional factors that may influence travel within a region. CSA is used as the generic name for a series of GIS techniques based on the ability to assign a cost to each cell in a raster map, and to accumulate these costs by travelling over the map. The least cost path over cost surface can be calculated over a non-network surface from a source cell to a destination cell.

Least cost path models in transportation date from the very earliest interest in GIS in the 1960s (Goodchild, 2000). These models considered the lowest travel cost-where cost is a function of time, distance, or other impedance factors-across terrain as an average value for a general area. Tobler was interested in the development of cost surface models through the calculation of the realistic time or cost distance in hilly terrain (Tobler, 1993). According to the empirical data, Tobler developed an equation to covert the calculated slope from terrain to walking velocity, which led to computation of the minimum time path from initial point to destination.

Balstrom used another approach by establishing a friction-based grid, which was derived from a combination of slope and a grid indicating rivers and lakes locations (Balstrom, 2002). This approach expressed the overall time needed to walk through a cell in the terrain based on field experiments. A cost surface was then calculated from one rain gauge at a time to all other rain gauges, establishing of network topology for the derived paths and ending with a vector network of least cost paths.

\subsection{Suitability Analysis}

Suitability analysis is a GIS-based process of determining the fitness of a given area for a particular use. The suitability model answers the question, "where is the best location?" For example, what is the best location for firebreaks, a new road, pipeline, or a new housing development?

Suitability analysis can be used for compliance with several elements to prepare comprehensive and general plans to identify constraints and opportunities for future land conservation and development (Steiner, McSherry, and Cohen, 2000). A project in the Appalachians Restoration Campaign examined the landscape in the central Appalachians and its suitability for supporting the eastern cougar (Taverna, Halbert and Hines, 2000).

\subsection{Web-Based GIS Application in Fire Emergency Projects}

Due to the rapid progress in information technology, the web-base GIS applications enhanced the ability to present spatial information analysis. Today, many types of GIS are available, ranging from the traditional GIS (for example, mainframe GIS, and Desktop GIS), distributed GIS (including Internet GIS and local environment network), and mobile GIS. A web-based GIS allows users to display spatial information on the Internet and wireless networks without installing GIS programs in the same desktop computer, unlike traditional GIS (Peng and Tsou, 2003).

A web-based GIS has been implemented in different fire emergency projects around the world. In 2002, the City of Fort Worth, Texas, began a project to create a user- 
friendly web interface to access and utilize the emergency operation center toolset, such as entering data, running reports, and performing numerous other functions. (Chatham \& Britton, 2002). In 2009, ESRINEA implemented a big project called a geographic security system, which consisted of different web applications such as an E-911web application that could capture the location of the fire incident from the call for service, assign classifications to the incident, and integrate with alarm systems (ESRINEA, 2009).

\subsection{Summary}

GIS could help the fire agencies to reduce the fire consequences, provide first responders with the best information to make optimized decisions to recover from fire quickly. In addition, the value of using GIS in FEM activities arises directly from the benefits of integrating a technology designed to support spatial decision making into a field with a strong need to address numerous critical spatial decisions. Using the CSA would help the fire agencies to reduce the response times to fires. The suitability analysis will provide the suitable location to prevent the fire and reduce its consequences (for example, the best location for firebreak).

A web-based GIS can play a key role as an efficient way to distribute the information to different users. The next chapter will outline the system analysis and design considerations for the project. 


\section{Chapter 3 - Systems Analysis and Design}

A successful GIS project involves understanding the needs of the client, the user, and the scope of the project to develop an effective plan for GIS implementation. A good project plan usually starts with a system design that will meet the client's expectations and minimize the negative impacts of various potential constraints. This chapter first revisits the problem that this project was trying to address. Next, it discusses three major components/units: the requirements analysis, system overview, and the development of the project plan. The requirements analysis describes the functional and non-functional requirements for the proposed GIS solution. The overview of the system design discusses the hardware and software needed for this project. Finally, the project plan captures the process to manage the tasks for this project.

\subsection{Problem Statement}

The JOTR was concerned with the threat of fires burning for hours or even days before the park's fire station is informed about them. Since there is not enough information about any fire incident, it was important to help the fire dispatch center assist JOTR with more rapid response to wildfire incidents. In addition, they do not know the shortest path from fire station to the fire incident, and they do not know the best place for firebreaks. A GIS solution was needed to help them identify the place where the fire incidents occur, to find the least cost path to the fire incident, and to find the best place to build firebreaks.

\subsection{Requirements Analysis}

This project focused on three major deliverables: two Python scripting tools that run a sequence of algorithms and calculate the cost surface, the final output of firebreak factors, and a web application that includes a set of functions to find the fire incidents locations and to run analysis and display the results in a user- friendly interface. To deliver these products to the client successfully, a requirements analysis was first conducted. The outcome of the requirements analysis was crucial for system design to meet the client's expectations, as well as resolve technical limitations. Accordingly, this section addresses both functional and non-functional (technical, operational, and transitional) requirements for this project.

\subsubsection{Functional Requirements}

Functional requirements outline the intended behavior of the system. They consider the user needs from a system perspective. Table 3-1 summarizes the functional requirements for the Cost Surface Analysis scripting tool. These requirements include the functionalities of the scripting tool that were identified considering potential use cases. The client needed to be able to prepare and convert the data to raster, and to use those data to calculate the cost surface for each fire station. Using the digital elevation model (DEM), roads and trails feature classes, as well as the fire stations feature class, the cost surface scripting tool, create the cost surface raster dataset. 
Table 3-1 Functional Requirements for the Cost Surface Analysis Scripting Tool

\section{Cost Surface Analysis Scripting Tool Requirements}

The tool must allow the user to select the DEM, roads, trails, and fire stations feature class as inputs to the tool.

The tool must be able to add speed field to the roads, trails feature class to allow the data classification.

The tool must be able to convert the DEM to slope.

The tool must be able to convert the speed of the roads and trails into time.

The tool must be able to calculate the cost surface for each fire station.

Similarly, Table 3-2 lists the functional requirements for the Firebreaks Factors scripting tool. These requirements address the functionalities of the suitability analysis that were identified considering potential use cases. The client needed to be able to prepare the data that used to locate the best places for firebreaks.

\section{Table 3-2 Functional Requirements for the Firebreak Factors Scripting Tool}

\section{Firebreak Factors Scripting Tool Requirements}

The tool should allow the user to select the inhabited structure features classes (such as campground), cultural sites feature classes (such as historic place), habitats feature classes (such as snakes), and vegetation feature class as inputs to the tool.

The tool should be able to combine the different feature classes to four factors: inhabited structures, cultural sites, habitats, and vegetation feature classes.

The tool should be able to combine all the four factors in one feature class.

Finally, Table 3-3 lists the functional requirements for the web application using ArcGIS API for Flex (Flex API). These requirements address the functionalities of the web application that were identified considering the potential use cases.

\section{Table 3-3 Functional Requirements for the Web Application}

\section{Web Application Requirements}

The web application must be able to locate the fire incident using different types of search (such as USGS names or coordinates system). 
The web application must be able to locate the least cost path from fire station to the incident.

The web application must be able to locate the best place for firebreaks.

\subsubsection{Non-functional Requirements}

Table 3-4 summarizes the non-functional requirements for the Cost Surface Analysis scripting tool, Firebreaks Factors scripting tool, and the web application. Non-functional requirements involved technical, operational, and transitional requirements that were determined based on potential use cases.

Table 3-4 Non-functional Requirements for FEMS

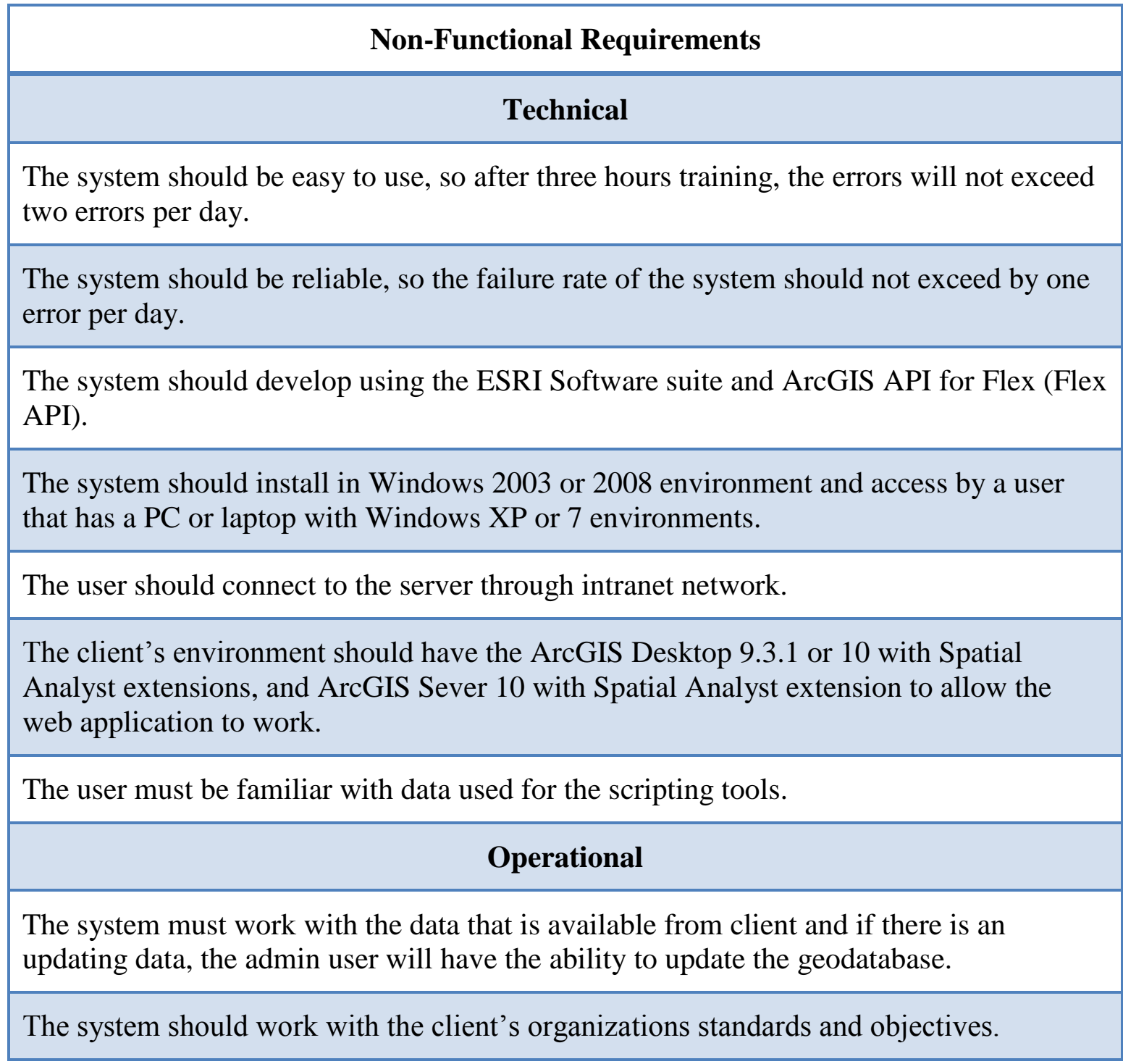


The system should host in client server that will prepare with ESRI software suite, windows server 2003or 2008, and the user will access the system through the organization intranet and workstation.

The administrator user should allow archiving the geodatabase daily or weekly

\section{Transitional}

A user tutorial will be prepared and given to the user during one day training that will explain the system functions and usability of the system.

The system should include an electronic version of user tutorial.

The web application and scripting tools should be tested before delivery.

The project team should be presented the project to JOTR fire station staff.

\subsubsection{Technical Requirements}

The technical requirements included the minimum software specifications necessary to use the two Python scripting tools and the web application. In order to run the Cost Surface Analysis scripting tool and Firebreaks Factors scripting tool, the client's computer needed ArcGIS Desktop 9.3.1 or higher, unlike the web application that required ArcGIS Server 10 with Spatial Analyst extension. In addition, the client's computer also must have the Spatial Analyst extension because the scripting tools include different spatial analysis tools that are available in the Spatial Analysis toolbox. This computing environment would enable the client to run the scripting tools to calculate the cost surface for each fire station, prepare the final output for firebreaks, and to run and display the analysis in the web application.

\subsubsection{Operational Requirements}

The operational requirements included the scripting tools and the web application that should work with the data that is available from the client. Additionally, the system should allow the client to archive the geodatabase.

\subsubsection{Transitional Requirements}

It is important that the user knows how to use the scripting tools and web application. An instruction manual will be provided to the client. Before product delivery, the system and scripting tools will be tested to ensure that it is functioning properly and producing the required outputs. One training session will be provided onsite to the client. The scripting tools and web application will be delivered to the client on a DVD. The project will be presented to the JOTR fire station staff at the same time the DVD and training are provided. 


\subsection{System Design}

The system was designed based on the outcome of the requirements analysis. It depicted the user needs and information product description in an interactive interface and described system design in different aspect maps, technology and system architecture.

\subsubsection{Maps}

Figure 3-1 was designed using the ArcMap and Spatial Analyst extension to build the geoprocessing tools used to identify the shortest path to the fire from a fire station, and to locate the best places for firebreaks. The map displays fire stations and the roads in JOTR to help the user determine the fire incident location.

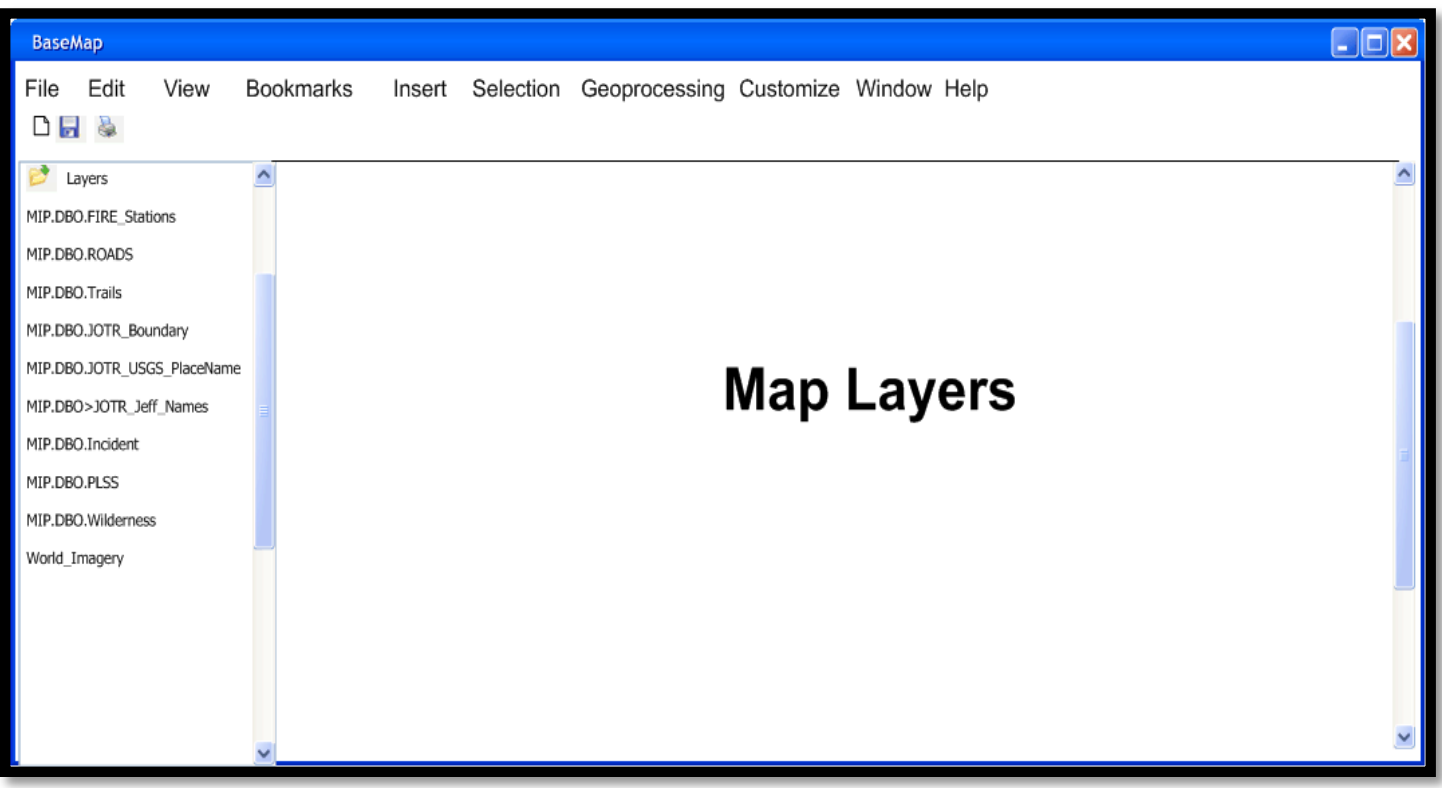

\section{Figure 3-1 FEMS Base Map}

Also, the development team built the base map to include the layers that the user will need. They used the scale range level to show some layers in a specific zooming level, which keeps the map from getting crowded, and helps the user to understand the map better (Figure 3-2). The information product description and user needs was a kick off step for building the base map, thinking how the map would satisfy the user's needs, and how it will work with the system functions. 


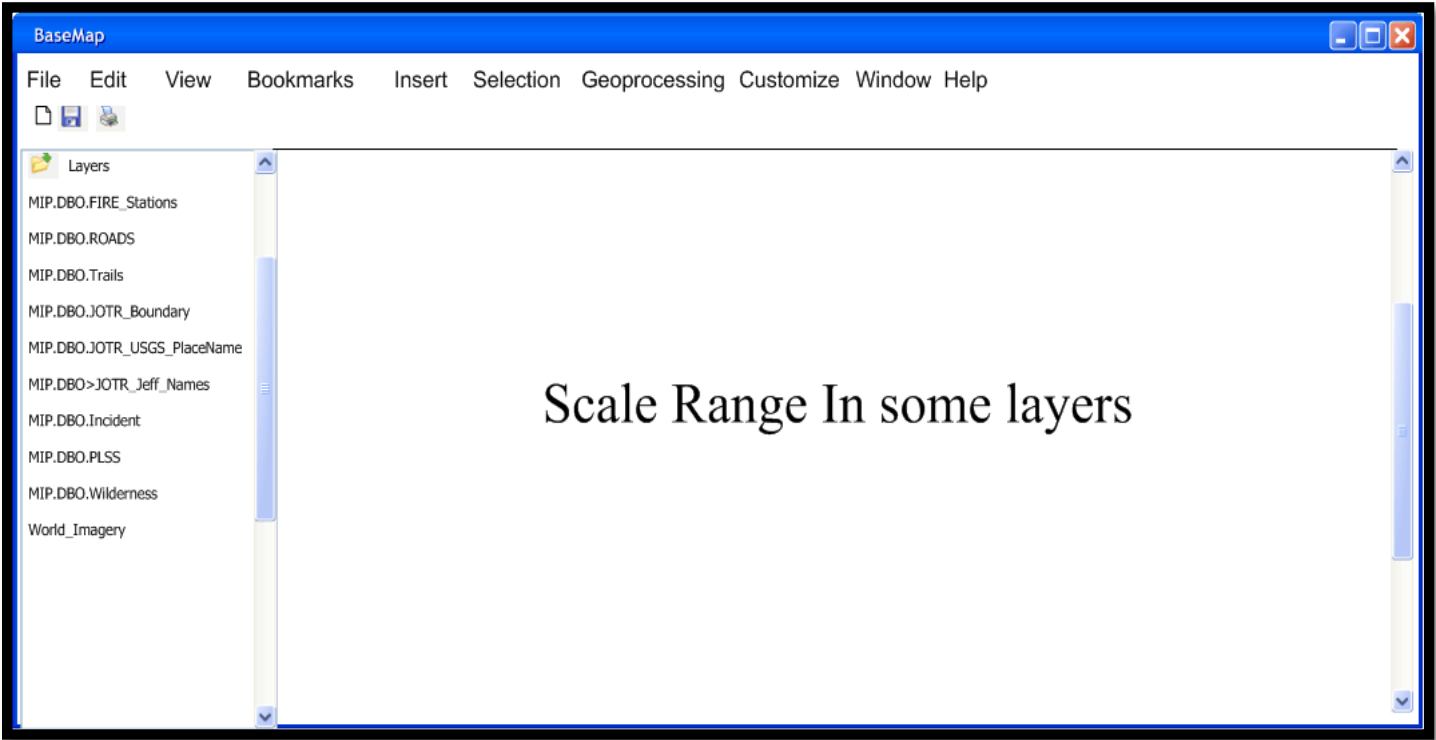

Figure 3-2 Scale Range in FEMS Base Map

\subsubsection{Technology}

Figure 3-3 was designed using API for Flex (Flex API), and ESRI software suite to build the base map, publish the services, and build the geodatabase. This system included different procedures and functions, such as locating the fire incident by using query to search about the USGS place-name and display it in the map. These procedures and functions depend on user needs and information product description, which is the successfuly key to build this system. 


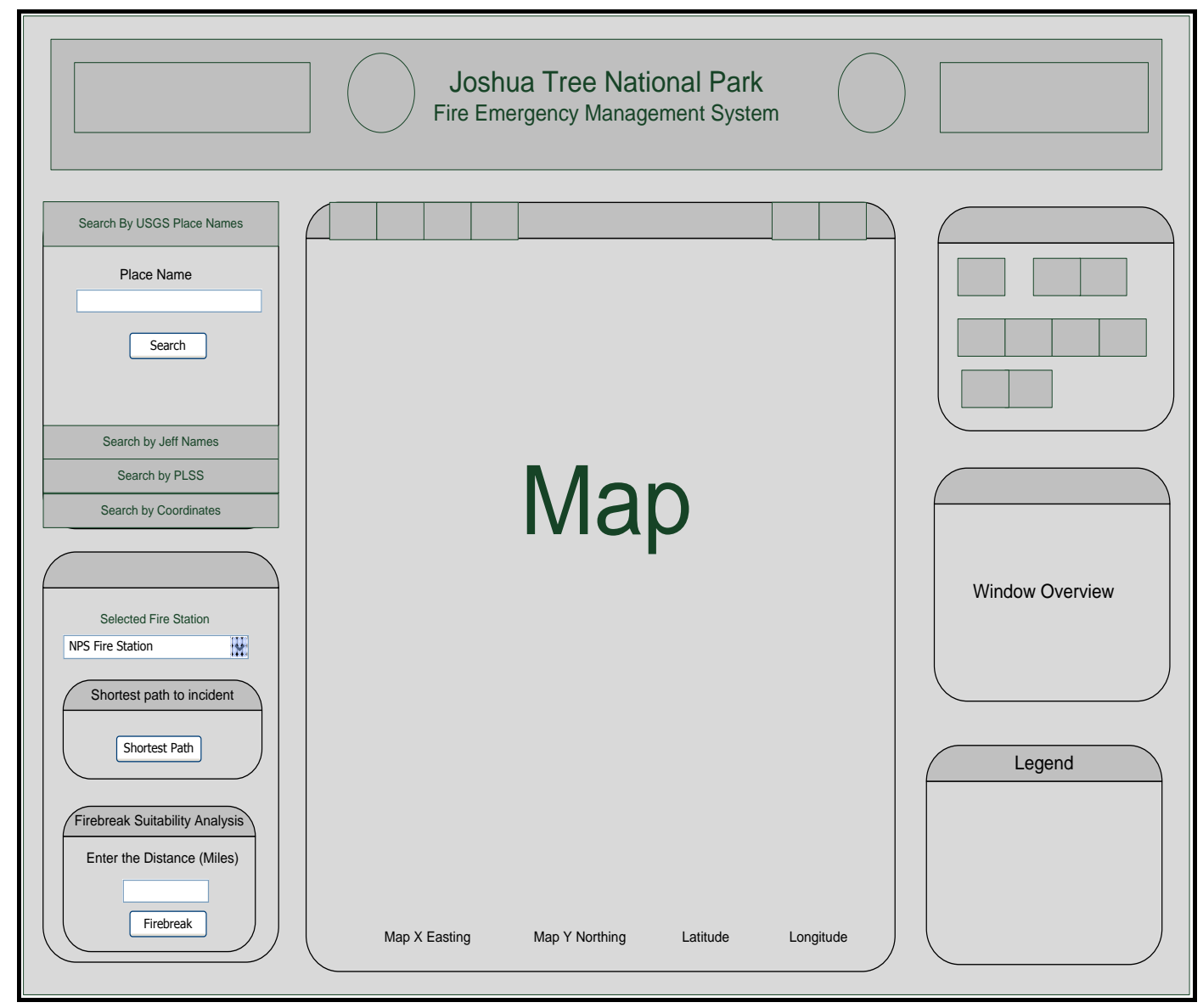

\section{Figure 3-3 FEMS Web Application Design}

The web application design depicted the user needs with simplicity of use and interactive interface. The development team designed the application to fit with user needs, and to implement the information product description.

\subsubsection{System Architecture}

Figure 3-4 displays an overview of the system architecture. The FEMS consists of two key tiers: client tier and GIS servers tier. Each component works in conjunction with ESRI's ArcGIS Info 10 (client tier can work with ESRI's ArcGIS Info 9.3.1) software suite, which encompasses all components of the system design).

The client tier consists of a workstation or laptop platform that will access the application through the web browser, while the GIS servers consists of internet information services (IIS), which handle the request through the web, and ArcGIS Server 10. In addition, ArcGIS Server 10 provides connectivity to geographic information through web services, and within that all maps and geoprocessing services are created and served through web services, which are consumed by the client. The server hosts the geoprocessing services, which run the spatial scripting tool and return the results to the client platform. 
The system architecture is a conceptual design that defines the structure of the system, which depends on the user needs from functions and the type of application. In addition, the web system structure is different from the desktop, as well as the system functions. 
System Architecture
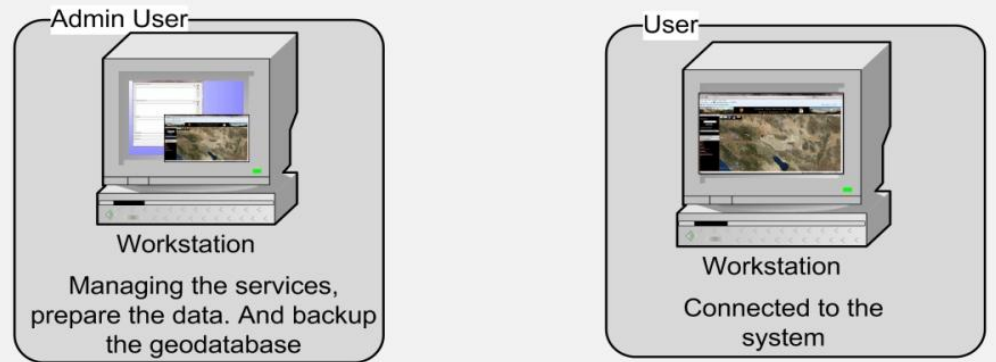

the geodatabase

system
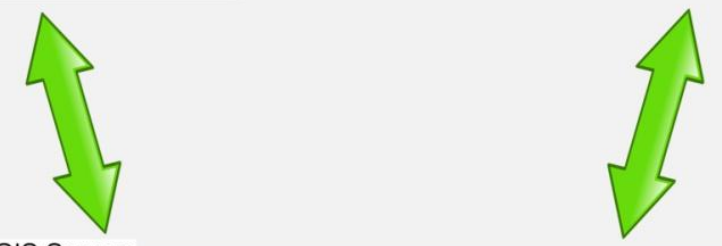

-GIS Servers

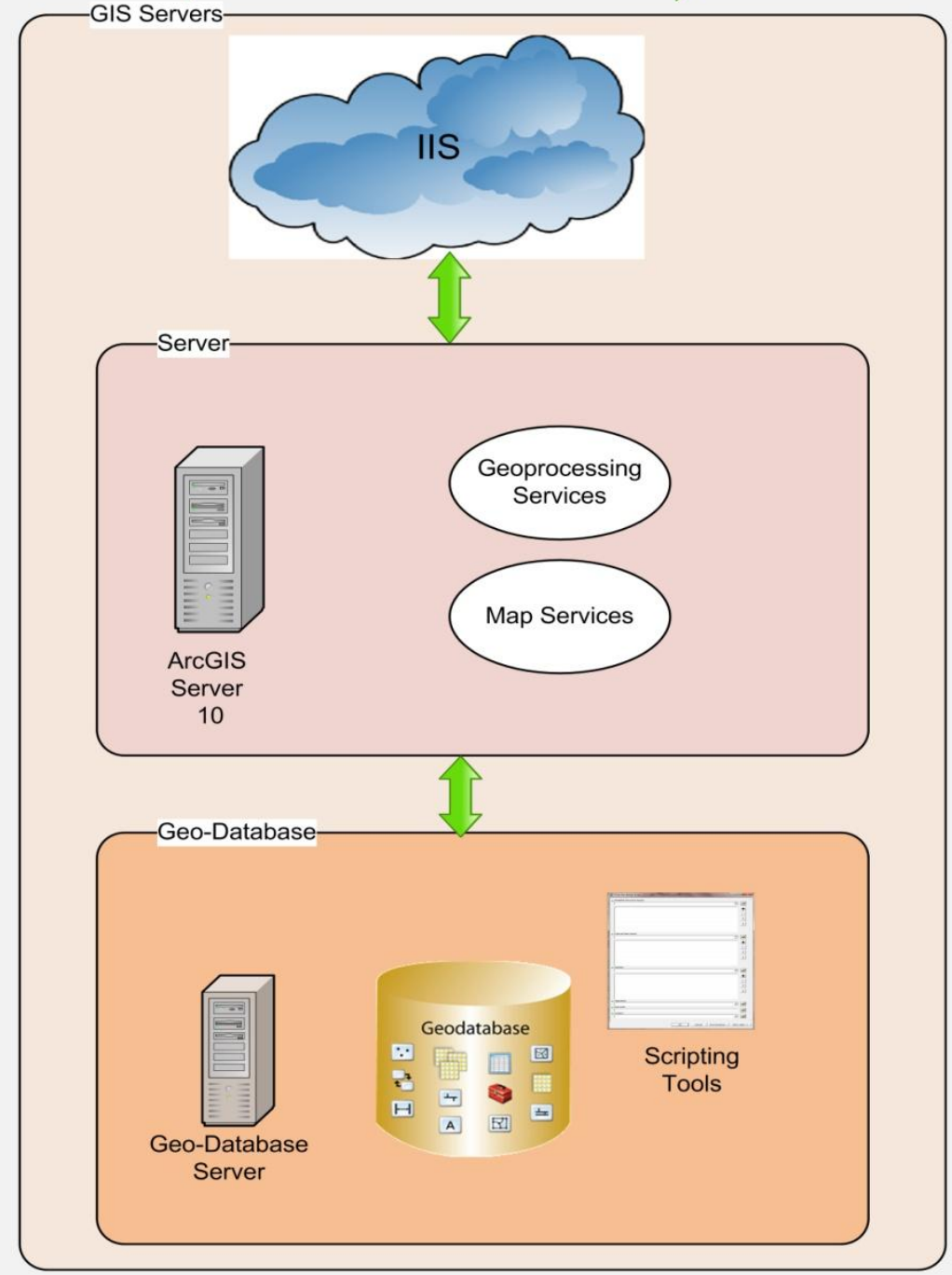

Figure 3-4 System Architecture 


\subsection{Project Plan}

To achieve the project's goals in a timely manner, the project plan initially consisted of six major phases, with an estimated time period needed to complete each task. (Figure 35) The initial proposed project plan was designed to accommodate the spiral method of project development and included:

- Phase 1: Defining the project

Meetings were conducted with the client to identify the problems the client was trying to address, the scope of the project, the goals and the solution, and the system process model.

- Phase2: Project Planning

Meetings were conducted with the client to collect the information needs through different processes, such as the interviews and questionnaire process, and assessed the project feasibility.

- Phase 3: System Analysis

Preliminary research was conducted in order to understand the functional and non-functional requirements. The research involved reviewing literature relevant to fire emergency management.

- Phase 4: System Design

This phase was to design a personal-ArcSDE geodatabase and feature dataset that incorporated the data, and to design the system and scripting tool interface.

- Phase 5: System Implementation and Testing

The web application was developed using the Flex API. The key functions included: identifying the fire incident location using different search types, identifying the shortest path to fire from a fire station, and to locating the best places for firebreaks. In addition, the scripting tools were built using a Python programming language. The key functions of scripting tools included calculating the cost surface from each fire station and preparing the firebreaks final output. Necessary modifications regarding the FEMS and scripting tools were discussed in order to meet the client's expectations.

- Phase 6: System Closing

The scripting tools, web application, and user documentation were compiled on a DVD. The project was presented at JOTR fire station. A poster of the project and a DVD with complete MIP report was delivered to the client. User training and presentation were delivered onsite at the JOTR.

The project was progressing from one phase to another dependent on the project plan, but not as the development team expected, since some tasks took longer than expected. For example, there some requirements were not clarified from the beginning, such as firebreak determination. In addition, designing a geodatabase task was not completed in time, because there were some data that are not available, such as PLSS data. Also, the development team did not adjust the project scope or schedule, but tried to complete the other requirements to fit within the project plan, and to find a time at the end to complete the project in a timely manner. There was a new requirements added, which needed more time to understand and complete. To complete the requirements within the time, the 
development team increased the number of working hours per day, and expanded the system implementation and testing phase by a week.

\begin{tabular}{|c|c|c|c|}
\hline ID & $\mathbf{B}$ & Task Name & Duration \\
\hline 1 & & Phase 1: Defining the project & 12 days? \\
\hline 2 & 田 & Project Selection & 3 days \\
\hline 3 & & Defining the problems of the client & 3 days \\
\hline 4 & 卧 & Defining the scope & 4 days \\
\hline 5 & & Defining the goals and the solutions & 3 days \\
\hline 6 & 匞 & Software Process Model selection & 1 day? \\
\hline 7 & 匞 & Defining project Document & 12 days \\
\hline 8 & & Phase 2: Project Planing & 20 days \\
\hline 9 & 匞 & Strategic Goals & 5 days \\
\hline 10 & & Information Needs & 5 days \\
\hline 11 & & Interviews & 3 days \\
\hline 12 & & Questionnaire & 2 days \\
\hline 13 & & Assessing Project Feasibility & 5 days \\
\hline 14 & 田 & Technical Feasibility & 2 days \\
\hline 15 & & Economic Feasibility & 2 days \\
\hline 16 & 臣 & Organizational Feasibility & 2 days \\
\hline 17 & 田 & Risk Analysis & 3 days \\
\hline 18 & 田 & Project Plan & 5 days \\
\hline 19 & 西 & Project Plan Document & 20 days \\
\hline 20 & & Phase 3: System Analysis & 50 days \\
\hline 21 & 榅 & Requirements Collection & 5 days \\
\hline 22 & & Software Requirements Specification & 5 days \\
\hline 23 & 画 & Designing Data Flow Diagram(DFD) & 4 days \\
\hline 24 & 臣 & Process Specification & 3 days \\
\hline 25 & 匞 & Designing Entity Relationship Diagram & 4 days \\
\hline 26 & 匞 & System Analysis Document & 22 days \\
\hline 27 & 田 & Requirements Clarification & 3 days \\
\hline 28 & 田 & Learn new Technology & 7 days \\
\hline 29 & & Phase 4: System Design & 26 days \\
\hline 30 & 匞 & Designing Database & 4 days \\
\hline 31 & & Designing The Web Application & 5 days \\
\hline 32 & 田 & Designing Scripting Tools & 3 days \\
\hline 33 & 卧 & System Design Document & 24 days \\
\hline 34 & 画 & Complete the geo-database design & 3 days \\
\hline 35 & & Phase 5:System Implementation And Testi & 50 days \\
\hline 36 & 匞 & System Implementation & 30 days \\
\hline 37 & & System Testing & 10 days \\
\hline 38 & 匞 & System Implementation and Testing Docume & 50 days \\
\hline 39 & & Phase 6: System closing & 14 days \\
\hline 40 & 臣 & System Installation & 3 days \\
\hline 41 & 臣 & System Training & 5 days \\
\hline 42 & 臣 & System User Manual Doucement & 8 days \\
\hline 43 & 卧 & System Documentation & 187 days \\
\hline
\end{tabular}

Figure 3-5 Preliminary Project Schedule 
When undertaking a project, there was always a risk area, which could affect successful, one-time completion, which is, understanding what the client needs, and what data is available. To avoid that, the development team expanded each phase by a week.

\subsection{Summary}

The overall system analysis and design, and project plan were discussed in this chapter. The requirements analysis was crucial for addressing the goals and objectives that the project was intended to achieve. The analysis was conducted focusing on both functional and non-functional requirements, including technical, operational, and transitional requirements. The overview of the system design discussed the hardware and software required for this project. An original project plan illustrated required tasks with their proposed milestones. Necessary changes were added to the initial project plan in order to complete the entire project in a timely manner. The next chapter will outline the database design for the project. 


\section{Chapter 4 - Database Design}

Every GIS system consists of a spatial database of geographic features and attributes information. The design of a GIS database is important because it helps to identify the data that are necessary for the project, explores data values, relationships and linkages, and organizes data as part of a system workflow. The database design also helps in identifying potential data problems and incorporates the functional needs of the system users. The development of the geodatabase for this project was straightforward because all the data came from JOTR in shapefile format.

This chapter discusses four key components of database design for the web-based application. First, Section 4.1 discusses the conceptual model, which aimed at defining significant entities of this project and representation associations between the entities. Second, Section 4.2 examines a logical data model which was required in order to implement the entities and associations represented by the conceptual data model. Third, Section 4.3 describes the data sources of this project. Finally, Section 4.5 discusses the data collection methods, and the data scrubbing and loading.

\subsection{Conceptual Data Model}

The conceptual data model comprises the entities of interest that address the project problems and their relationships. This design model provides a structures layout of the components of the project, which facilitates data organization and determination of a system workflow. Important tasks involved in creating a conceptual model includes: identification of data objects or entities, investigation of the corresponding attributes, and identification of the data relationships. In addition, a conceptual data model is a highlevel abstraction that illustrates the complex real world in a simplistic way. There are three unified model language (UML) diagrams illustrating the relationship of use cases in a relational database that are relevant to this project. Figure 4-1 illustrates the relationships of the entire system conceptual data model. 


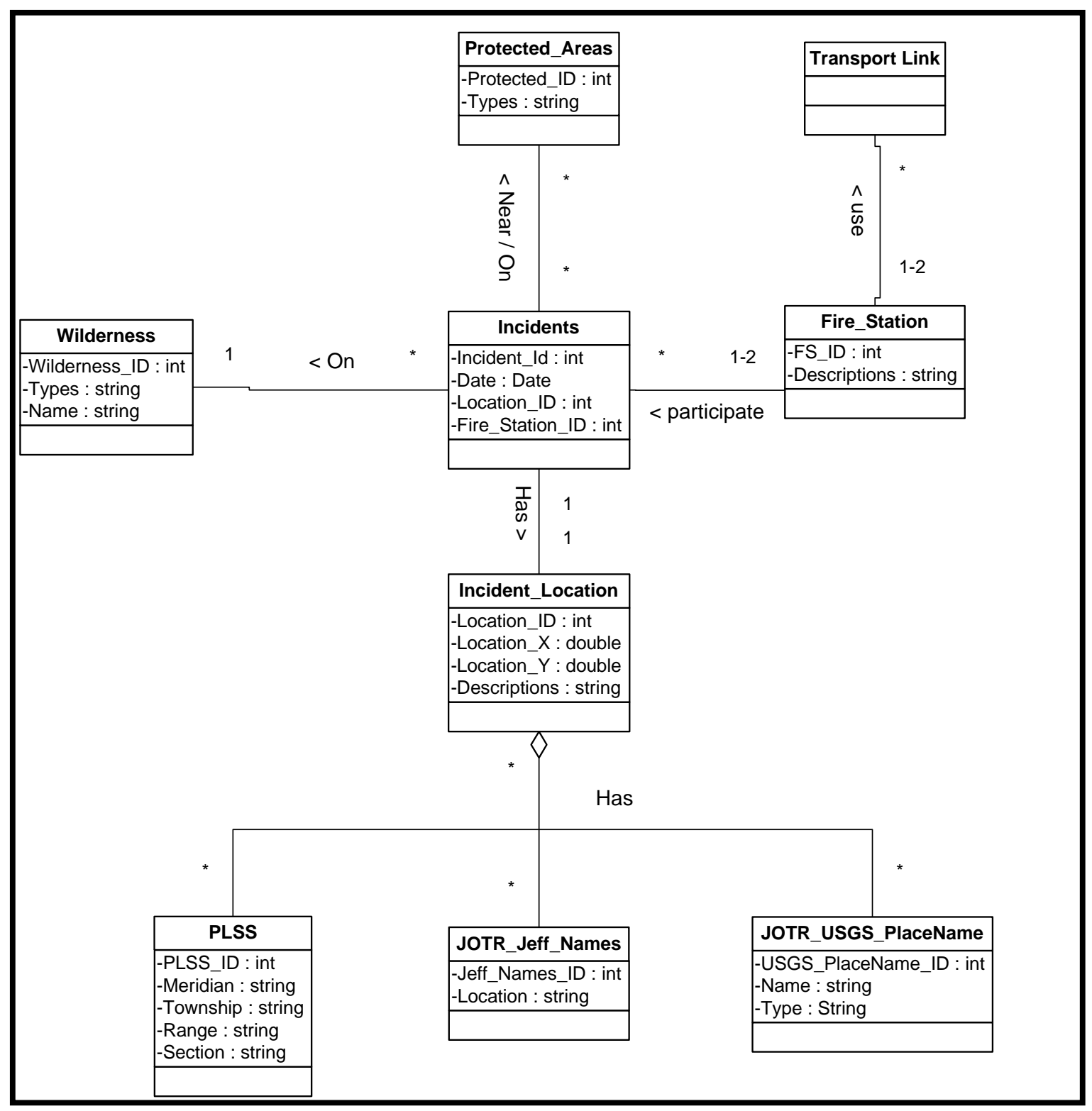

\section{Figure 4-1 System Conceptual Data Model}

This model illustrates the relationship between the Incidents entity with the other entities such as: Incident Location, Wilderness, and Protected Areas. Within this model, an incident can have a location that could be a PLSS location, place name location, coordinates location, or JOTR local names location (Jeff names). The association relationship between these entities is many-to-many. For example, an incident can locate in many PLSS locations, as a PLSS location can locate many incidents. In addition, an incident can locate on a wilderness area, as the incident can locate near or on the protected areas. The association relationship between the wilderness area and incident is one-to-many, which means many incidents can be on the wilderness. The association relationship between protected areas and incidents is many-to-many, which means many incidents can locate near or on one or many protected areas. A fire station can participate 
in an incident, and use the transport link to reach the fire. The association relationship between these entities is one-to-many, which means one or two fire stations can participate in the incidents and use many transport link. Further thought on this conceptual diagram led to a more in-depth look at the construction of protected areas, and the response to fire incident. This is depicted in Figures 4-2 and 4-3.

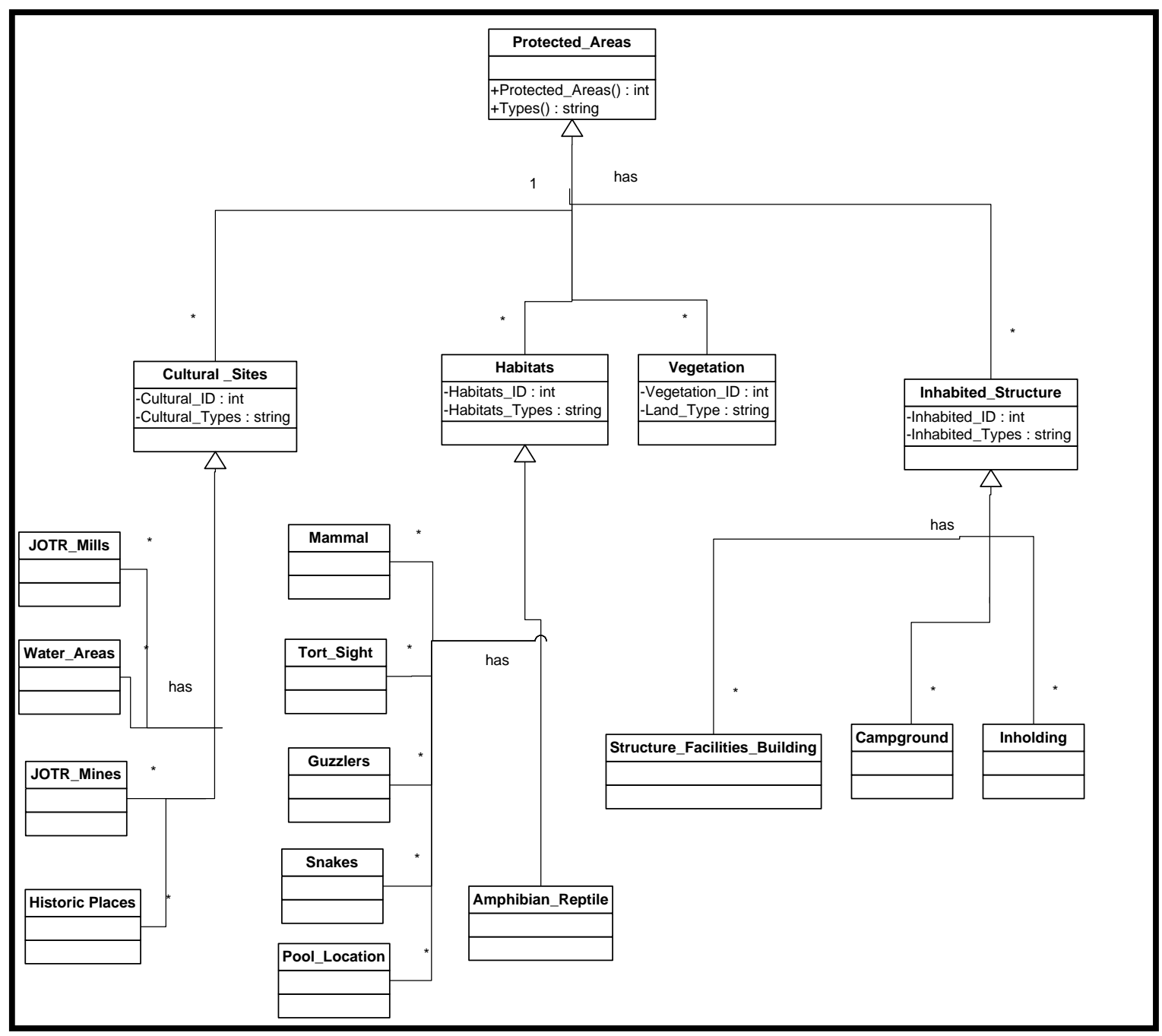

Figure 4-2 Protected Area Conceptual Data Model

Figure 4-2 illustrates the relationship between the Protected Areas entity with the other entities: Inhabited Structures, Habitats, Cultural Sites, and Vegetation. Within this model, inhabited structures, habitats, cultural sites, or vegetation are parts of the protected areas entity, while the structures facilities building, campground, and holding are parts of the inhabited structures entity. The association relationship between these entities is oneto-many. For example, an inhabited structure can have many structure facilities buildings, many campgrounds, and many inholding areas. In addition, mammals, tortoises, guzziers (water holes for sheep), snakes, and pool locations are part of the 
habitats entity, as mills, mines, historic places, and water areas are part of cultural sites entity. The association relationship between these entities is one-to-many.

In Figure 4-3, roads, trails, and least cost path are part of a transport link entity.

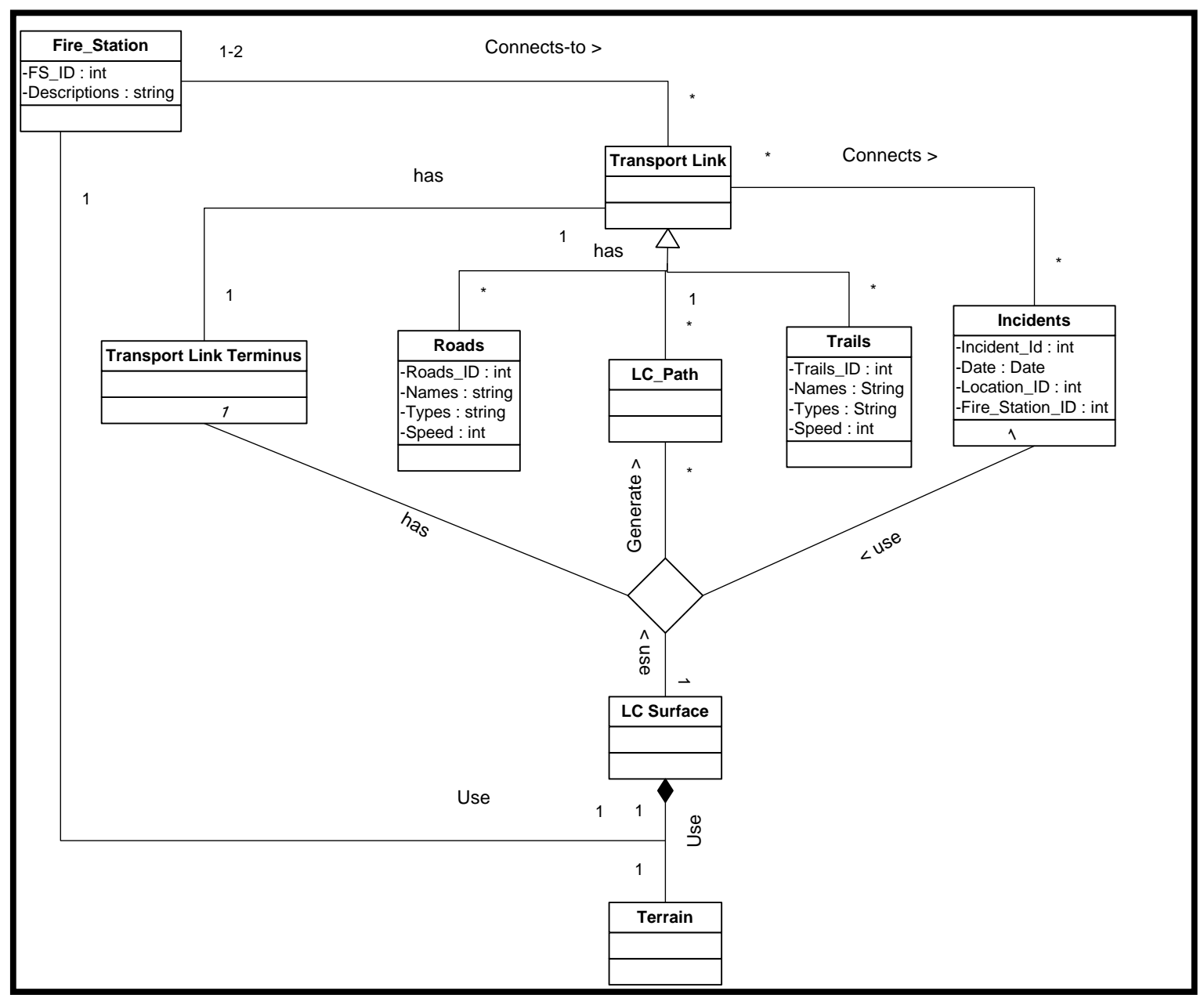

Figure 4-3 Response to Fire Conceptual Data Model

The association relationship between these entities is one-to-many. This means, a transport link consists of many roads or many trails or both of them. The transport link has many least cost paths, and a transport link terminus. The association relationship is one-to-one, which means one transport link has two termini. Many least cost paths generated using a cost surface, an incident, and transport link terminus entities. The association relationship between these entities is one-to-many. The Least Cost Surface raster dataset was created for each fire station using a terrain. The association relationship between these entities is one-to-one. A fire station can connected to an incident through the transport link entity. 


\subsection{Logical Data Model}

The logical data model takes the conceptual model and moves it into the physical geodatabase design phase. Tomlinson stated "a logical data model not only represents the data in computer logic but also describes the data in terms the computer can virtually "understand"- the model sets up its version of the real world with all its rules and orders that your data must follow" (Tomlinson, 2007, pg 93-94). However, a personal-ArcSDE geodatabase was chosen for this project because it has the ability to provide for feature classes that would be edited or synchronized from a the web application to be stored. An ArcSDE is required for any layers or feature classes that are dynamically updated from web application because of the editing and permission rules in the personal-ArcSDE.

Within ArcEditor and ArcInfo, the ArcCatalog application provides the ability to fully administer and manage the personal-ArcSDE geodatabase using SQL Server Express. This provides full personal-ArcSDE geodatabase capabilities for several users at a time with a limited geodatabase size to $4 \mathrm{~GB}$. A geodatabase was used in this project mainly for organization purposes and data integrity, providing a central location for easy data access and management. A geodatabase often contains multiple feature classes and the feature classes that have similar characteristics are frequently grouped together in feature datasets within the geodatabase.

The context of this project, a logical data model, was created to describe a personal-ArcSDE geodatabase with two datasets, and a set of raster datasets (Figure 4-4).

\begin{tabular}{|c|c|c|}
\hline Contents & Preview Description & \\
\hline \multicolumn{2}{|l|}{ Name } & Type \\
\hline \multicolumn{2}{|c|}{ 直MIP.DBO.FireBreak } & SDE Feature Dataset \\
\hline \multicolumn{2}{|c|}{ MIP.DBO.BaseMap } & SDE Feature Dataset \\
\hline \multicolumn{2}{|c|}{ MIP.DBO.BASEMAPHILSHIDE } & SDE Raster Dataset \\
\hline \multicolumn{2}{|c|}{ MIP.DBO.BLM_BACKLINKK } & SDE Raster Dataset \\
\hline \multicolumn{2}{|c|}{ “网 MIP.DBO.BLM_TRAVELTIME } & SDE Raster Dataset \\
\hline \multicolumn{2}{|c|}{ MIP.DBO.NPS_BACKLINK } & SDE Raster Dataset \\
\hline \multicolumn{2}{|c|}{ আ MIP.DBO.NPS_TRAVELTIME } & SDE Raster Dataset \\
\hline \multicolumn{2}{|c|}{ MIP.DBO.ROADSRASTER } & SDE Raster Dataset \\
\hline \multicolumn{2}{|c|}{ MIP.DBO.ROADSTRAILS } & SDE Raster Dataset \\
\hline \multicolumn{2}{|c|}{ MIP.DBO. SLOPE } & SDE Raster Dataset \\
\hline \multicolumn{2}{|c|}{ MIP.DBO.TRAVELCOST } & SDE Raster Dataset \\
\hline \multicolumn{2}{|c|}{ MIP.DBO.TRAVELSPEED } & SDE Raster Dataset \\
\hline
\end{tabular}

\section{Figure 4-4 GeodataBase Feature Datasets}

These raster datasets were used to prepare the Cost Surface feature classes, and then used those features to find the least cost path to a fire incident. Figure 4-5 illustrates the Firebreaks dataset that consists of different point and polygon feature classes. These feature classes were used to prepare the final polygon feature class used in the firebreak analysis. 


\begin{tabular}{|c|c|c|}
\hline Contents & Preview Description & \\
\hline \multicolumn{2}{|l|}{ Name } & Type \\
\hline \multicolumn{2}{|c|}{$\because$ MIP.DBO.Water_Areas } & SDE Feature Class \\
\hline \multicolumn{2}{|c|}{$\because$ MIP.DBO.tort_sight } & SDE Feature Class \\
\hline \multicolumn{2}{|c|}{ (2) MIP.DBO.Vegetation } & SDE Feature Class \\
\hline \multicolumn{2}{|c|}{$\because$ MIP.DBO.snakes } & SDE Feature Class \\
\hline \multicolumn{2}{|c|}{$\because$ MIP.DBO.Structure_Building } & SDE Feature Class \\
\hline \multicolumn{2}{|c|}{$\because$ MIP.DBO.Pool_locations } & SDE Feature Class \\
\hline \multicolumn{2}{|c|}{$\because$ MIP.DBO.mammal } & SDE Feature Class \\
\hline \multicolumn{2}{|c|}{$\because$ MIP.DBO.JOTR_Mines } & SDE Feature Class \\
\hline \multicolumn{2}{|c|}{$\because$ MIP.DBO.JOTR_Mills } & SDE Feature Class \\
\hline \multicolumn{2}{|c|}{ MIP.DBO.inholding } & SDE Feature Class \\
\hline \multicolumn{2}{|c|}{$\because$ MIP.DBO.Historic_Places } & SDE Feature Class \\
\hline \multicolumn{2}{|c|}{$\because$ MIP.DBO.guzzler } & SDE Feature Class \\
\hline \multicolumn{2}{|c|}{$\because$ MIP.DBO.campground } & SDE Feature Class \\
\hline \multicolumn{2}{|c|}{$\because$ MIP.DBO.Amphibian_Reptile } & SDE Feature Class \\
\hline \multicolumn{2}{|c|}{ 품. MIP.DBO.Habitats } & SDE Feature Class \\
\hline \multicolumn{2}{|c|}{ (․⿹ㅁ MIP.DBO.Habitats_Dissolve } & SDE Feature Class \\
\hline \multicolumn{2}{|c|}{ [:- MIP.DBO.Inhabited_Structure } & SDE Feature Class \\
\hline \multicolumn{2}{|c|}{ (19) MIP.DBO.Inhabited_Dissolve } & SDE Feature Class \\
\hline \multicolumn{2}{|c|}{ (i) MIP.DBO.Cultural_Sites } & SDE Feature Class \\
\hline \multicolumn{2}{|c|}{ [i⿻日禸;)MIP.DBO.Cultural_Dissolve } & SDE Feature Class \\
\hline \multicolumn{2}{|c|}{ 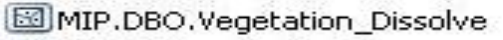 } & SDE Feature Class \\
\hline \multicolumn{2}{|c|}{ [: MIP.DBO.protected_Areas } & SDE Feature Class \\
\hline
\end{tabular}

\section{Figure 4-5 Firebreak Dataset}

Figure 4-6 illustrates the BaseMap dataset that consists from set of point and polygon feature classes.

\begin{tabular}{|c|c|c|}
\hline Contents & Preview Description & \\
\hline \multicolumn{2}{|l|}{ Name } & Type \\
\hline \multicolumn{2}{|c|}{ [國MIP.DBO.JOTR_Bounday } & SDE Feature Class \\
\hline \multicolumn{2}{|c|}{$\because$ MIP.DBO.JOTR_Jeff_Names } & SDE Feature Class \\
\hline \multicolumn{2}{|c|}{-OMIP.DBO.JOTR_USGS_PlaceNames } & SDE Feature Class \\
\hline \multicolumn{2}{|c|}{ MIP.DBO.Wilderness } & SDE Feature Class \\
\hline \multicolumn{2}{|c|}{ 國MIP.DBO.PLSS } & SDE Feature Class \\
\hline \multicolumn{2}{|c|}{$\succeq$ MIP.DBO. Trails } & SDE Feature Class \\
\hline \multicolumn{2}{|c|}{$\mp$ MIP.DBO.ROADS } & SDE Feature Class \\
\hline \multicolumn{2}{|c|}{$\because$ MIP.DBO.Fire_Stations } & SDE Feature Class \\
\hline \multicolumn{2}{|c|}{$\because$ MIP.DBO.Incidents } & SDE Feature Class \\
\hline
\end{tabular}

\section{Figure 4-6 BaseMap Dataset}




\section{Table 4-1 Feature Class Attributes Data Types}

\begin{tabular}{|l|l|l|}
\hline Feature Class & Attributes Name & Data Types \\
\hline \multirow{4}{*}{ Incidents } & Incident_ID & Integer \\
\cline { 2 - 3 } & Date & Date \\
\cline { 2 - 3 } & Fire_Station_ID & Integer \\
\cline { 2 - 3 } & Status & String \\
\cline { 2 - 3 } & Description & String \\
\hline
\end{tabular}

Table 4-1 illustrates the attributes and data types for Incidents spatial database engine (SDE) feature class. There is an association relationship between Incidents and Fire Station SDE feature classes. The Fire_Station_ID is a primary key in the Fire_Station SDE feature class and foreign key in the Incidents SDE feature class. The Incidents SDE feature class has different attributes, such as Incident_ID, which is a primary key, Date to store the incident date, Status to check the incident is steal open or close, and the Description to describe the incident.

\subsection{Data Sources}

Sean Murphy, the GIS specialist at JOTR, provided the data for this project. However, it is necessary to give an idea of the data source that is served as a part of the project. The data was projected in the NAD 1983 UTM Zone $11 \mathrm{~N}$ projection system and most of the data that came from the client were in shapefile format. In addition, the feature loaded into the personal-ArcSDE geodatabase had metadata included either with the feature class or from text files imported by the NPS Metadata Editor. Apart from some features, such as guzzler and water areas shapefiles, this did not have any metadata. Table 4-2 summarizes the data sources as the data came from the client.

\section{Table 4-2 Data Source Summary}

\begin{tabular}{|l|l|l|l|}
\hline \multicolumn{1}{|c|}{ Feature } & \multicolumn{1}{|c|}{ Description } & \multicolumn{1}{|c|}{ Format } & \multicolumn{1}{c|}{$\begin{array}{c}\text { Metadata } \\
\text { availability }\end{array}$} \\
\hline Fire_Station & $\begin{array}{l}\text { Fire_Station is a } \\
\text { point shapefile that } \\
\text { depicting the fire } \\
\text { station of JOTR } \\
\text { and Mojave desert } \\
\text { that will used if } \\
\text { there is a fire in } \\
\text { the park }\end{array}$ & Shapefile & None \\
\hline jeff_names & $\begin{array}{l}\text { This shapefile } \\
\text { depicts points in } \\
\text { the park that Jeff } \\
\text { Ohlfs (Longtime }\end{array}$ & Shapefile & Available in text \\
file
\end{tabular}




\begin{tabular}{|c|c|c|c|}
\hline & $\begin{array}{l}\text { park ranger) } \\
\text { thought would be } \\
\text { of use to others }\end{array}$ & & \\
\hline pbd09_poly & $\begin{array}{l}\text { It is a shapefile } \\
\text { polygon to depict } \\
\text { the JOTR } \\
\text { boundary as of } \\
2009\end{array}$ & Shapefile & $\begin{array}{l}\text { Available with } \\
\text { the shapefile }\end{array}$ \\
\hline drg_name & $\begin{array}{l}\text { This point } \\
\text { shapefile shows } \\
\text { all words that are } \\
\text { printed on USGS } \\
7.5 \text { minute maps } \\
\text { covering JOTR }\end{array}$ & Shapefile & $\begin{array}{l}\text { Available in text } \\
\text { file }\end{array}$ \\
\hline PLSS & $\begin{array}{l}\text { This shapefile } \\
\text { shows section lines } \\
\text { in and around } \\
\text { JOTR }\end{array}$ & Shapefile & $\begin{array}{l}\text { Available in text } \\
\text { file }\end{array}$ \\
\hline Roads and public roads & $\begin{array}{l}\text { These polylines } \\
\text { shapefiles show } \\
\text { the roads in JOTR }\end{array}$ & Shapefile & $\begin{array}{l}\text { Available in text } \\
\text { file }\end{array}$ \\
\hline Trails & $\begin{array}{l}\text { This polyline } \\
\text { shapefile shows } \\
\text { the trails in JOTR }\end{array}$ & Shapefile & $\begin{array}{l}\text { Available in text } \\
\text { file }\end{array}$ \\
\hline Wilderness & $\begin{array}{l}\text { Shows areas } \\
\text { within JOTR that } \\
\text { are required to be } \\
\text { managed as } \\
\text { wilderness and } \\
\text { these areas } \\
\text { included potential } \\
\text { wilderness, } \\
\text { proposed } \\
\text { wilderness, and } \\
\text { full wilderness }\end{array}$ & Shapefile & $\begin{array}{l}\text { Available in text } \\
\text { file }\end{array}$ \\
\hline campg & $\begin{array}{l}\text { This point } \\
\text { shapefile that } \\
\text { shows the location } \\
\text { of the campground } \\
\text { area }\end{array}$ & Shapefile & $\begin{array}{l}\text { Available in text } \\
\text { file }\end{array}$ \\
\hline Historic & $\begin{array}{l}\text { This point } \\
\text { shapefile that } \\
\text { shows the location } \\
\text { of historic area }\end{array}$ & Shapefile & $\begin{array}{l}\text { Available in text } \\
\text { file }\end{array}$ \\
\hline Guzzler & This point & Shapefile & Available in text \\
\hline
\end{tabular}




\begin{tabular}{|c|c|c|c|}
\hline & $\begin{array}{l}\text { shapefile that } \\
\text { shows the water } \\
\text { holes for big horn } \\
\text { sheep }\end{array}$ & & file \\
\hline Jtmine & $\begin{array}{l}\text { This point } \\
\text { shapefile shows all } \\
\text { the mines known } \\
\text { to exist within } \\
\text { JOTR }\end{array}$ & Shapefile & $\begin{array}{l}\text { Available in text } \\
\text { file }\end{array}$ \\
\hline Mills2 & $\begin{array}{l}\text { This point } \\
\text { shapefile shows } \\
\text { the location of } \\
\text { abandoned mill } \\
\text { site in JOTR }\end{array}$ & Shapefile & $\begin{array}{l}\text { Available in text } \\
\text { file }\end{array}$ \\
\hline Inholding & $\begin{array}{l}\text { This polygon } \\
\text { shapefile shows } \\
\text { parcels of land } \\
\text { within JOTR that } \\
\text { are not owned by } \\
\text { the USA } \\
\text { government }\end{array}$ & Shapefile & $\begin{array}{l}\text { Available in text } \\
\text { file }\end{array}$ \\
\hline$A \& r$ & $\begin{array}{l}\text { This point } \\
\text { shapefile shows } \\
\text { the location of } \\
\text { amphibian and } \\
\text { reptile habitats in } \\
\text { JOTR }\end{array}$ & Shapefile & $\begin{array}{l}\text { Available in text } \\
\text { file }\end{array}$ \\
\hline Mammal & $\begin{array}{l}\text { This point } \\
\text { shapefile shows } \\
\text { the location of } \\
\text { mammal habitats } \\
\text { in JOTR }\end{array}$ & Shapefile & $\begin{array}{l}\text { Available in text } \\
\text { file }\end{array}$ \\
\hline Snakes & $\begin{array}{l}\text { This point } \\
\text { shapefile shows } \\
\text { the location of } \\
\text { snakes habitats in } \\
\text { JOTR }\end{array}$ & Shapefile & $\begin{array}{l}\text { Available in text } \\
\text { file }\end{array}$ \\
\hline Tort_sight & $\begin{array}{l}\text { This shapefile } \\
\text { shows the point } \\
\text { data where desert } \\
\text { tortoises have been } \\
\text { seen in the park }\end{array}$ & Shapefile & $\begin{array}{l}\text { Available in text } \\
\text { file }\end{array}$ \\
\hline $\begin{array}{l}\text { Pool locations - } \\
\text { Smithwater Canyon }\end{array}$ & $\begin{array}{l}\text { This point } \\
\text { shapefile shows } \\
\text { the pool location } \\
\text { in JOTR }\end{array}$ & Shapefile & None \\
\hline
\end{tabular}




\begin{tabular}{|c|c|c|c|}
\hline Water & \begin{tabular}{|l|} 
This point \\
shapefile shows \\
the water areas \\
location in JOTR \\
\end{tabular} & Shapefile & None \\
\hline building_centroids_Project & $\begin{array}{l}\text { This polygon } \\
\text { shapefile is a } \\
\text { component of the } \\
\text { prototype } \\
\text { implementation } \\
\text { plan for the NPS } \\
\text { Buildings data } \\
\end{array}$ & Shapefile & $\begin{array}{l}\text { available in text } \\
\text { file }\end{array}$ \\
\hline Vegetation & $\begin{array}{l}\text { This feature class } \\
\text { created by Aerial } \\
\text { Information } \\
\text { Systems (AIS) for } \\
\text { JOTR in the late } \\
\text { 1990's and this } \\
\text { feature class serves } \\
\text { as the final version } \\
\text { of the vegetation. }\end{array}$ & Feature class & $\begin{array}{l}\text { available within } \\
\text { the feature class }\end{array}$ \\
\hline DEM 10m & $\begin{array}{l}\text { This is a raster } \\
\text { dataset that shows } \\
\text { the digital } \\
\text { elevation model } \\
\text { for JOTR within } \\
10 \mathrm{~m}\end{array}$ & Raster & $\begin{array}{l}\text { Available within } \\
\text { the raster dataset }\end{array}$ \\
\hline World imagery & $\begin{array}{l}\text { This layer show } \\
\text { the world imagery } \\
\text { layer }\end{array}$ & Satellite image & None \\
\hline US Topo layer & $\begin{array}{l}\text { This layer shows } \\
\text { the US } \\
\text { topographic map } \\
\text { layer }\end{array}$ & Topographic map & None \\
\hline
\end{tabular}

\subsection{Data Scrubbing and Loading}

A substantial portion of the project consisted of the preparation of data for loading into the personal-ArcSDE geodatabase. The process is outlined in details in Chapter 5, which describes the implementation phase of the project. There were two main reasons for data scrubbing and loading the data: the preparation of feature classes to accommodate with firebreak priority analysis and cost surface analysis, and to organize the data in the geodatabase.

Data provided by JOTR required very little editing prior to loading it into the geodatabase. For example, add a new field (speed) in the roads and trails feature classes to accommodate with cost surface analysis. Before loading the shapefiles to geodatabase, the metadata for all feature classes were loaded, and a feature datasets was created to 
organize the data in the geodatabase. Moreover, roads feature class was created using public roads and roads features classes, because both features have similar data. The repeated data was deleted from some shapefiles such as the USGS Places Names shapefile.

\subsection{Summary}

In this chapter, the principles of what constitutes a good database design were discussed. A conceptual data model, often represented as a UML diagram, was first constructed to plan for the necessary data and workflow of the project. The conceptual data model illustrated the foremost entities of the FEMS application and important associations between those entities. The logical data model was created in order to implement the entities and associations represented by the conceptual data model. Prior to this analysis, the primary inputs were examined by content and modified to meet the needs of the cost surface and firebreak priority analysis. Finally, the different data sources for the project were discussed. The next chapter will outline the implementation methods. 



\section{Chapter 5 - Implementation}

Chapter Five discusses the project implementation, which includes generating the cost surface to find the shortest path to the fire from a fire station, preparing the data for firebreaks priority analysis, creating geoprocessing scripts to display the analysis results on the web, and locating the fire incident location using different types of search methods. The first two tasks were completed by creating a geoprocessing scripting tools using Python. The last two tasks were fulfilled by creating a web application using ArcGIS API for Flex (Flex API) development framework and Python to display the analysis results on the web.

\subsection{Cost Surface Analysis Scripting Tool}

The cost surface scripting tool was created using Python scripting language in order to perform a sequence of algorithms. This tool requires inputs such as: roads feature class, trails feature class, fire stations feature class, DEM raster dataset, and the data path folder (Figure 5-1).

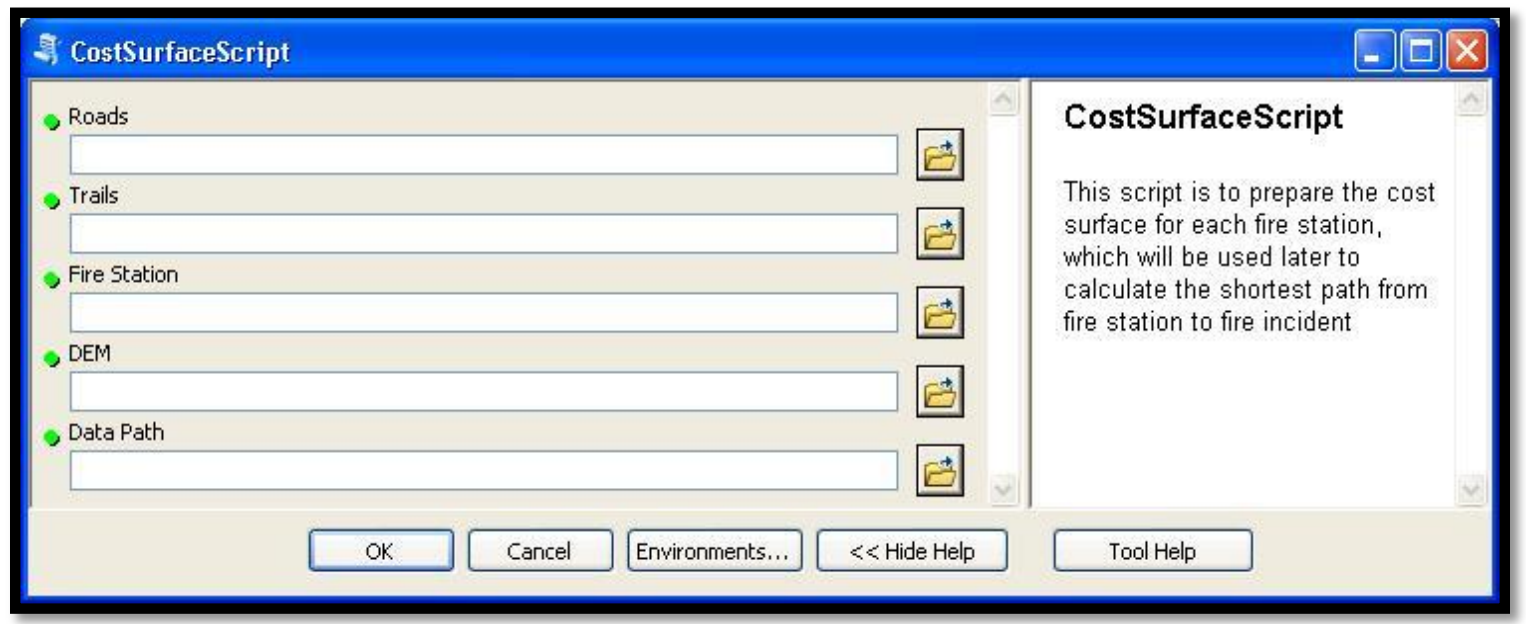

Figure 5-1 Cost Surface Analysis Scripting Tool

All the outputs of this tool were stored in a personal-ArcSDE geodatabase. This section outlines the overall functions of the Cost Surface Analysis tool. This tool was used to prepare the primary inputs for cost surface analysis. Creating the Cost Distance depends on the fire stations and Cost Travel raster dataset that were prepared before. Figure 5-2 shows the overall workflow for generating the cost surface. 


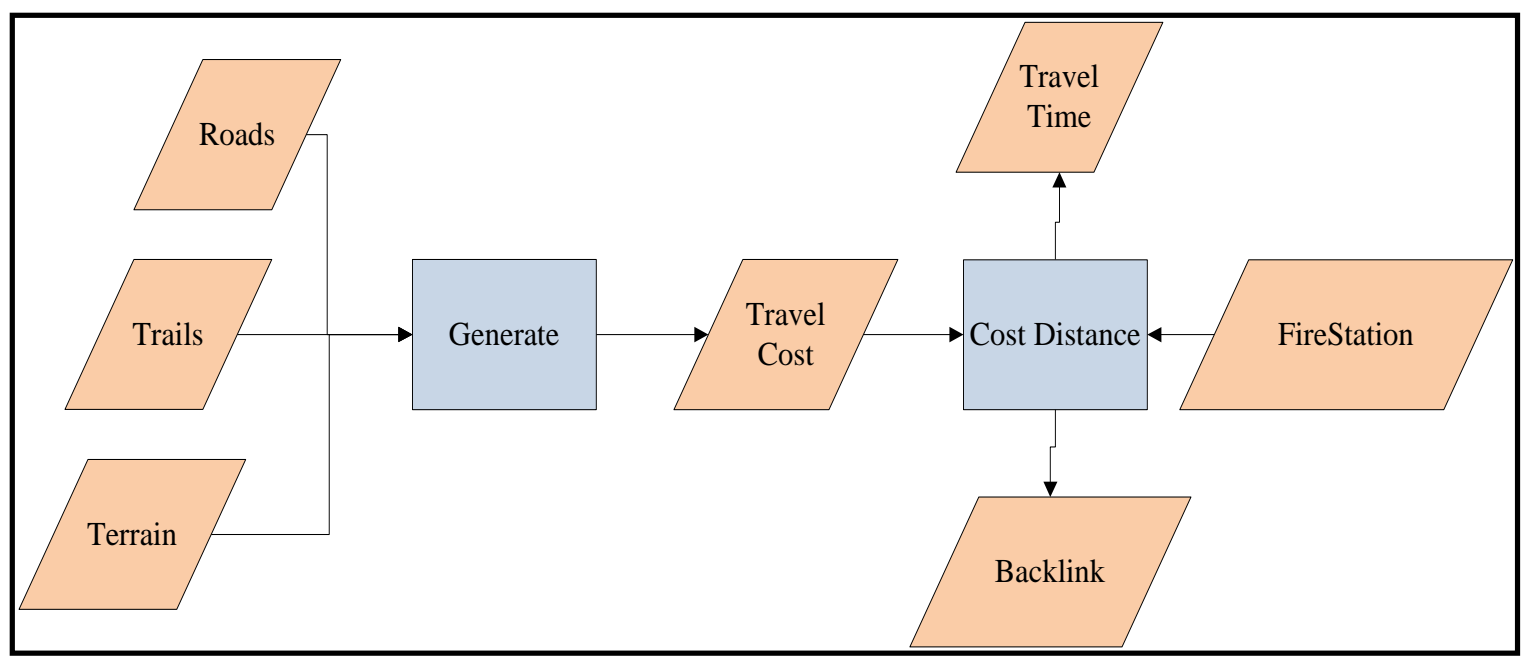

\section{Figure 5-2 Cost Surface Analysis Workflow}

\subsubsection{Preparing the Primary Inputs}

The Cost Surface Analysis first required Roads, Trails, and Terrain feature classes to be prepared to generate the Travel Cost surface, and then run the Cost Distance tool that will be discussed in the next subsection. There are a set of steps to prepare the Roads and Trails feature classes (Figure 5-3).

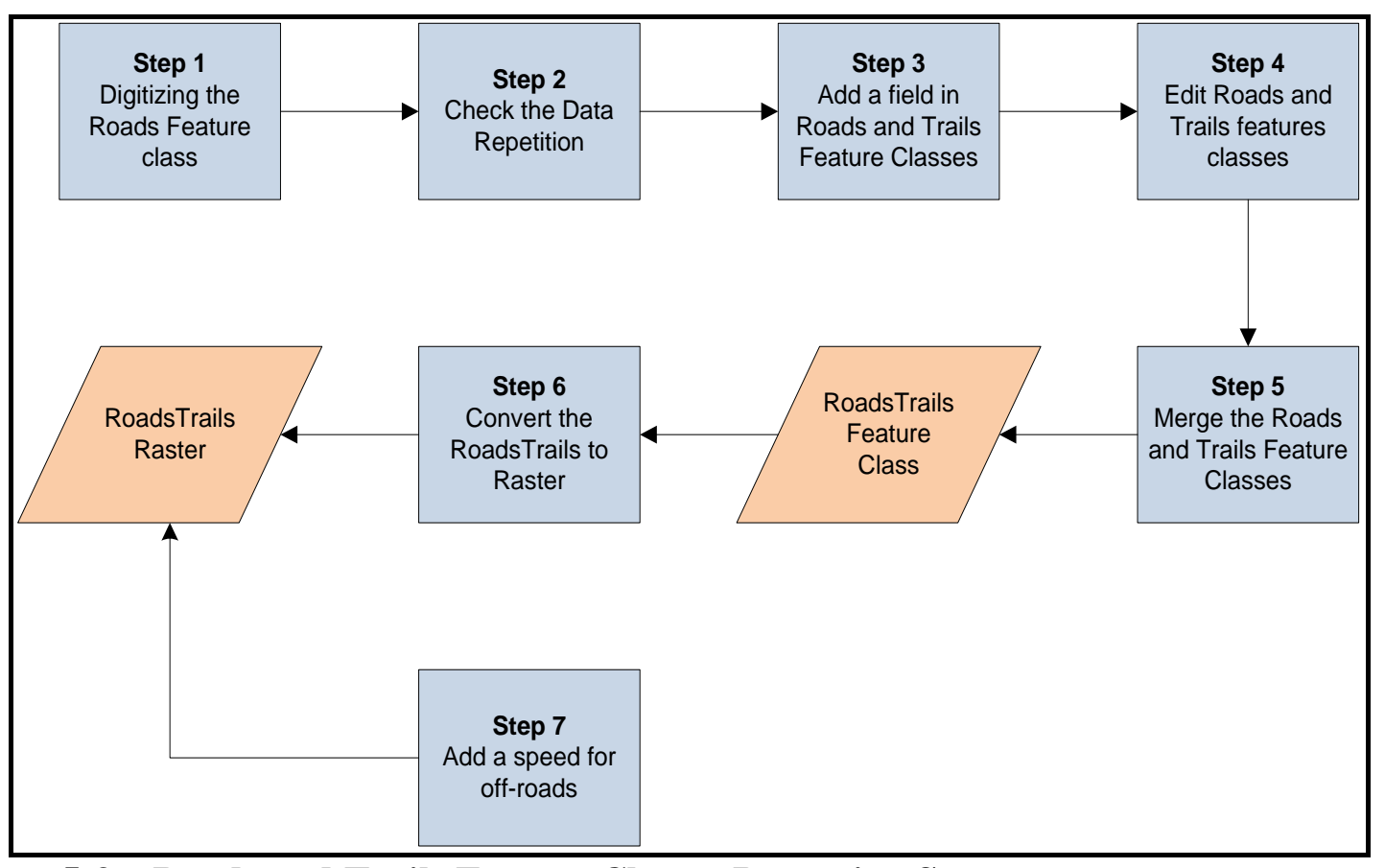

Figure 5-3 Roads and Trails Feature Classes Preparing Steps 
First, the Roads and Public-Roads feature classes have similar data, so the development team decided to digitize the Roads feature class with the missing PublicRoads, and create a final Roads feature class (Figure 5-4).

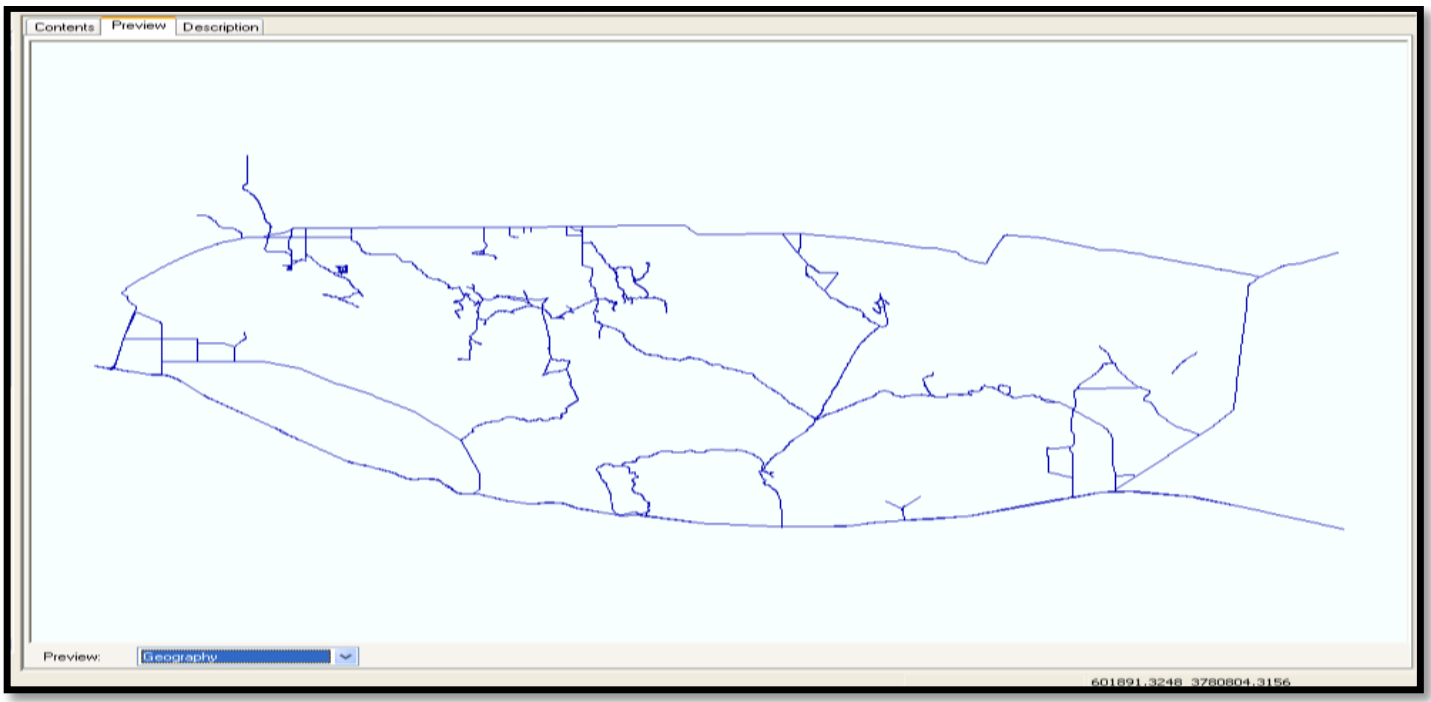

\section{Figure 5-4 Final Roads Feature Class}

Second, the data records in both Roads and Trails feature classes were checked to avoid data repetition. Moreover, a field was added in both the Roads and Trails feature classes to use in the analysis. The development team discussed the speed of different roads types and trails with the client and the project advisor to finalize the speed. The final speed parameters are listed in Table 5-1.

\section{Table 5-1 Road and Trail Speeds}

\begin{tabular}{|c|c|}
\hline Roads Types & Speed \\
\hline Paved & 50 miles per hours (MPH) \\
\hline Dirt & $35 \mathrm{MPH}$ \\
\hline Dirt 4X4 & $20 \mathrm{MPH}$ \\
\hline Trails & $3 \mathrm{MPH}$ \\
\hline
\end{tabular}

The roads and trails feature classes were then edited using the editing toolbar and field calculator function in ArcMap (Figures 5-5 and 5-6). 


\begin{tabular}{|c|c|c|c|c|c|}
\hline ROADS_HAME & ROADS_TYPE & Speed & Shape * & Shape.len & $\sqrt{\wedge}$ \\
\hline JOSHUA LANE & PAVED & 50 & Polyline & 405.238626589 & \\
\hline TMENTYYNINE PALMS HIGHWAY & PAVED & 50 & Polyline & 8186.19374812 & \\
\hline SAN MARINO DRIVE & PAVED & 50 & Polyline & 81.3247525135 & \\
\hline SAN MARINO DRIVE & PAVED & 50 & Polyline & 771.078523267 & \\
\hline SAN MARINO DRIVE & PAVEU & 50 & Poryline & 26.3447721467 & \\
\hline BLACK ROCK CANYON & PAVED & 50 & Polyline & 425.359367783 & \\
\hline GOLD CROMNN ROAD & DIRT $4 \times 4$ & 20 & Polyline & 2243.65125998 & \\
\hline BLACK ROCK CANYON & PAVED & 50 & Polyline & 389.029648415 & \\
\hline BLACK ROCK CAMPGROUND ROADS & DIRT & 35 & Polyline & 18.9625829701 & \\
\hline BLACK ROCK CAMPGROUND ROADS & DIRT & 35 & Polyline & 79.7763166590 & \\
\hline BLACK ROCK CAMPGROUND ROADS & DIRT & 35 & Polyline & 259.536027253 & \\
\hline BLACK ROCK CAMPGROUND ROADS & DIRT & 35 & Polyline & 20.4263433450 & \\
\hline BLACK ROCK CAMPGROUND ROADS & DIRT & 35 & Polyline & 697.575154347 & \\
\hline BLACK ROCK CAMPGROUND ROADS & DIRT & 35 & Polyline & 95.1792968947 & \\
\hline BLACK ROCK CAMPGROUND ROADS & DIRT & 35 & Polyline & 154.93232252 & \\
\hline BLACK ROCK CAMPGROUND ROADS & DIRT & 35 & Polyline & 317.987971413 & \\
\hline BLACK ROCK CAMPGROUND ROADS & DIRT & 35 & Polyline & 125.183722394 & \\
\hline
\end{tabular}

\section{Figure 5-5 Roads Editing}

\begin{tabular}{|c|c|c|c|}
\hline SOURCE & Speed & Shape * & Shape-len \\
\hline original & 3 & Polyline & 136.02840538322 \\
\hline original & 3 & Polyline & 248.928729714875 \\
\hline original & 3 & Polyline & 1788.61515162776 \\
\hline original & 3 & Polyline & 524.180713701709 \\
\hline original & 3 & Polyline & 1021.80043171181 \\
\hline original & $\sqrt{3}$ & Ponyline & 620.678841089631 \\
\hline original & 3 & Polyline & 531.248977860679 \\
\hline original & 3 & Polyline & 598.388118630578 \\
\hline original & 3 & Polyline & 1672.34551973911 \\
\hline original & 3 & Polyline & 709.450669086869 \\
\hline original & 3 & Polyline & 322.506422460999 \\
\hline original & 3 & Polyline & 335.444230316196 \\
\hline original & 3 & Polyline & 536.64179511621 \\
\hline original & 3 & Polyline & 623.717259588595 \\
\hline original & 3 & Polyline & 765.941157296588 \\
\hline original & 3 & Polyline & 1332.188924986 \\
\hline original & 3 & Polyline & 285.179374689514 \\
\hline original & 3 & Polyline & 1081.6597904978 \\
\hline original & 3 & Polyline & 178.26204373684 \\
\hline original & 3 & Polyline & 2313.62160515399 \\
\hline original & 3 & Polyline & 1067.42026690254 \\
\hline original & 3 & Polyline & 1019.37981752531 \\
\hline original & 3 & Polyline & 931.19543121717 \\
\hline original & 3 & Polyline & 1469.20105679581 \\
\hline original & 3 & Polyline & 75.85224205335 \\
\hline original & 3 & Polyline & 2743.4730830357 \\
\hline Loricinal & 3 & Polyline & 933.722852989935 \\
\hline
\end{tabular}

\section{Figure 5-6 Trails Editing}

The Cost surface scripting tool merged the Roads and Trails feature classes using the Merge function syntax (Table 5-2).

Table 5-2 Road and Trails Functions Syntax

\begin{tabular}{|l|l|}
\hline \multicolumn{1}{|c|}{ Function } & \multicolumn{1}{c|}{ Scripting Code } \\
\hline Merge & gp.Merge_management("'" + ROADS+"';" + Trails + "', \\
& "in_memoryllROADS_Trails_Merge", "Speed 'Speed' true true \\
& false 2 Short 00 ,First,\#,"+ROADS+",Speed,-1,- \\
& 1,"+Trails+",Speed,-1,-1") \\
\hline FeatureToRaster & gp.FeatureToRaster_conversion("in_memoryl|Roads_Trails_M \\
\hline
\end{tabular}




\begin{tabular}{|l|l|}
\hline & erge", "Speed", finalData+ "IIMIP.sdelIRoadsRaster", "30") \\
\hline $\begin{array}{l}\text { SingleOutputMapAlge } \\
\text { bra }\end{array}$ & gp.SingleOutputMapAlgebra_sa("Con(IsNull(C:IIdatalIFinal \\
& MIPIIMIP.sdelIMIP.DBO.ROADSRASTER),2,C:IIdatal\Final \\
& MIPIIMIP.sdelIMIP.DBO.ROADSRASTER)", finalData+ \\
& "IIMIP.sdelIRoadsTrails", "'" + finalData+ \\
& "IIMIP.sdelIMIP.DBO.ROADSRASTER'") \\
\hline
\end{tabular}

This function was used to combine inputs features from multiple inputs sources of the same data type (point, or line, or polygon) into a single output feature class, and then was used as an input in another function (Figure 5-7).

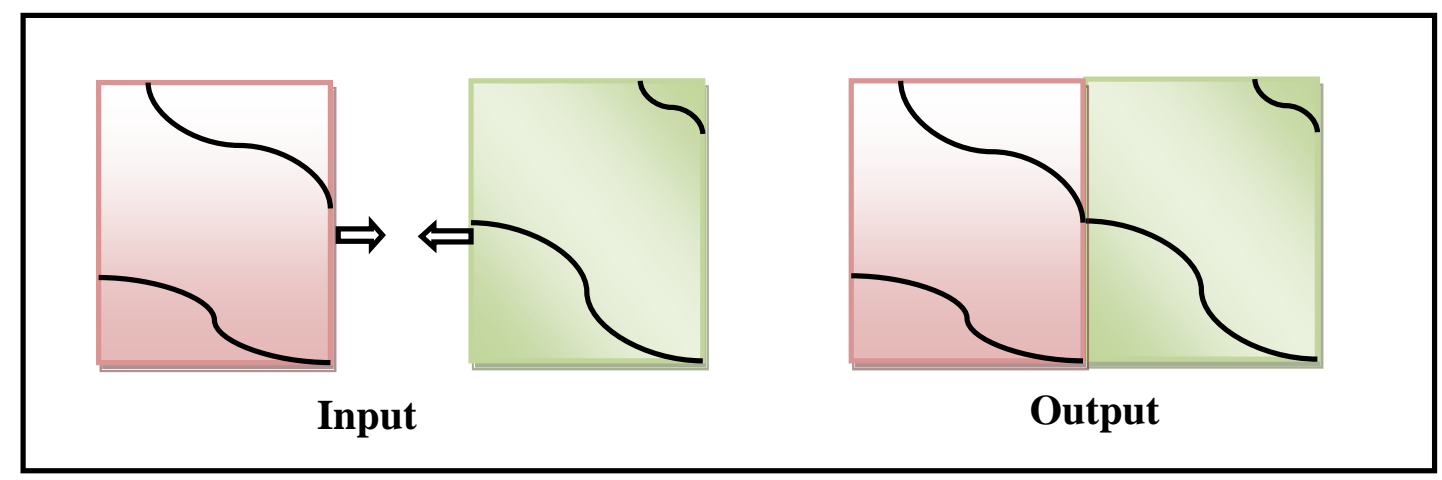

Figure 5-7 Merge Function (ESRI, ArcGIS 9.3.1 Help)

The tool created the Roads_Trails_Merge feature class in server memory using the syntax "in_memoy\Roads_Trails_Merge". In addition, in the Merge function syntax, the development team specified the fields needed to display in the output feature such as speed field.

The output of Merge function was used as an input for the FeatureToRaster function, which is a function under the conversion toolset in ArcToolbox. This function was used to convert features to a raster dataset and used a field to assign values to the output raster (Table 5-2). The input for this function was retrieved from the server memory using the syntax "in_memory $\backslash$ Roads_Trails_Merge" and used the speed field to assign the values to the output raster (Figure 5-8).

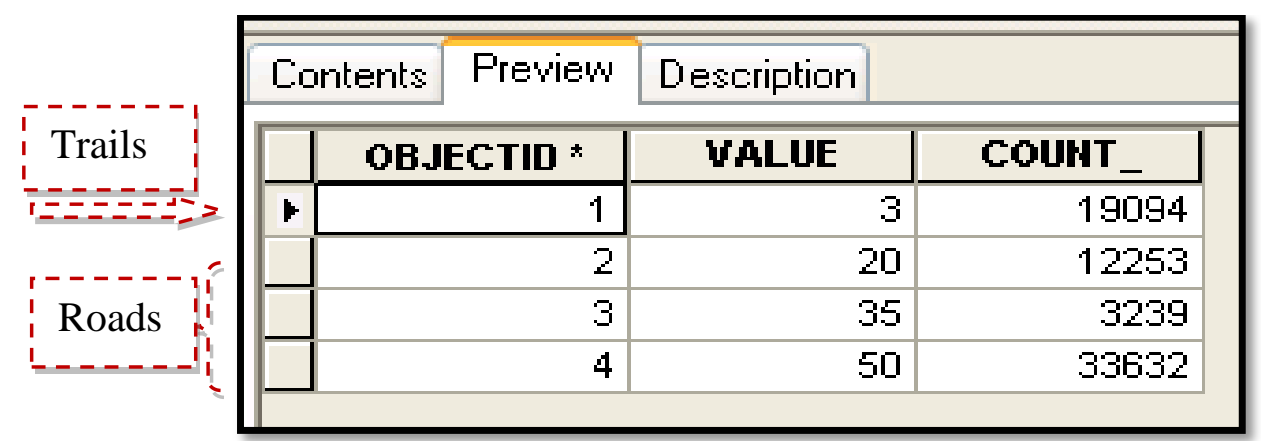

Figure 5-8 The Output of FeatureToRaster Function 
The default cell size is the smallest of the width or height of the extent of input feature, in the output spatial reference, divided by 250 map units. The cell size of the output raster was changed to 10 meters to make the resolution as the DEM resolution. The output raster of FeatureToRaster function was assigned speed limit values of 3 if it is trail or 20,35, 50 if it is one of the road types. The cells between the RoadsTrails were assigned the no-data value. The development team assumed 2MPH of speed for off-road after discussion with the client and the advisor. Map Algebra was then used to replace the no-data cells with the off-road speed limited to 2. To use the Map Algebra function, the Spatial Analyst extension must be present. Table 5-2 shows the Map Algebra syntax, which mean, if the cell value of RoadRaster is no-data, make the output cell value 2; otherwise, leave it as the value of RoadRaster and create an output raster called RoadsTrails (Figure 5-9).

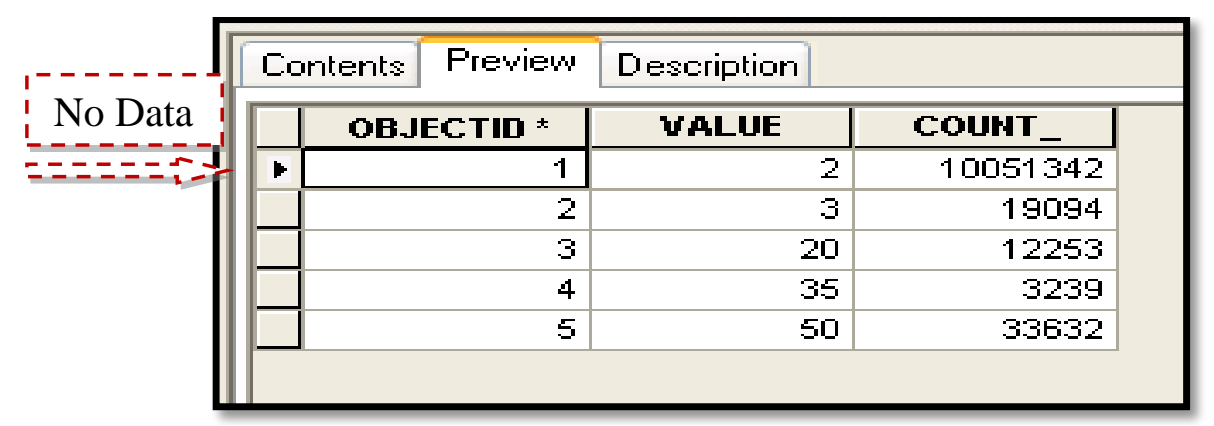

\section{Figure 5-9 The Output of Map Algebra}

Figure 5-10 shows the overall steps to generate the slope and create the travel cost surface. Every cell now has a speed limit value, and the off-road speed limit is the same for all terrain types, so the development team adjusted the off-road speed limits based on the slope of the terrain.

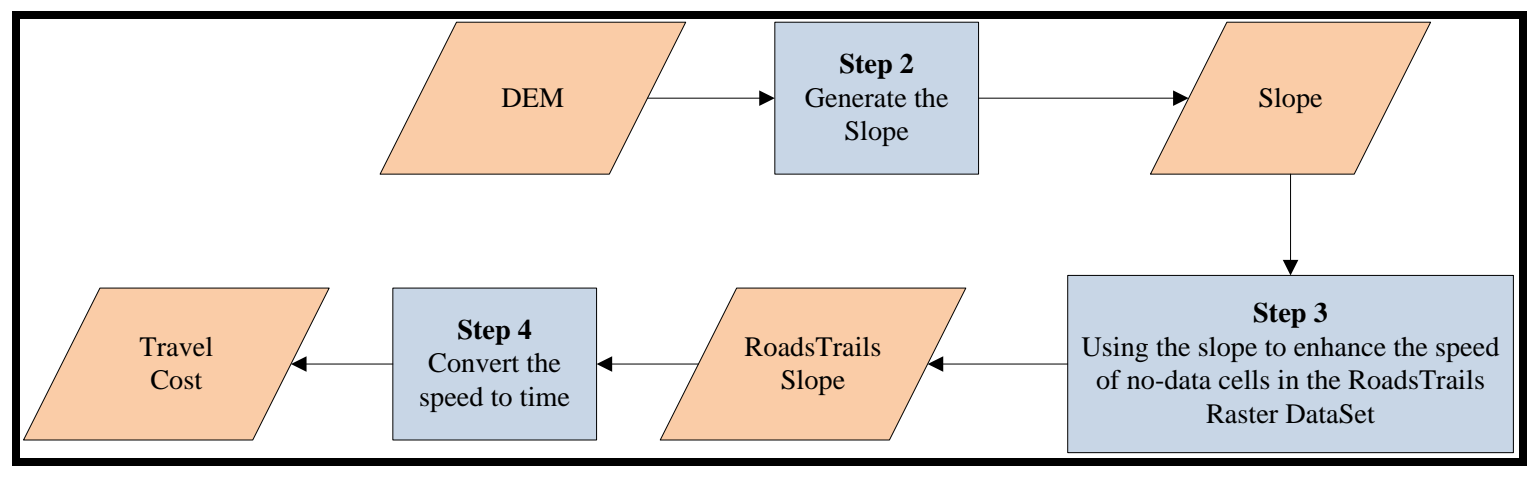

Figure 5-10 Overall Steps for Creating the Travel Cost Surface 
Table 5-3 DEM Function Syntax

\begin{tabular}{|l|l|}
\hline \multicolumn{1}{|c|}{ Function } & \multicolumn{1}{c|}{ Scripting Code } \\
\hline Slope & $\begin{array}{l}\text { gp.Slope_sa(DEM, finalData+ } \\
\text { "IIMIP.sdellSlope", "DEGREE", "1") }\end{array}$ \\
\hline
\end{tabular}

Table 5-3 shows the syntax of Slope function, which identify the rate of maximum change in z-value from each cell, and accepts a raster (DEM) as an input and a raster dataset as an output raster dataset (Figure 5-11).

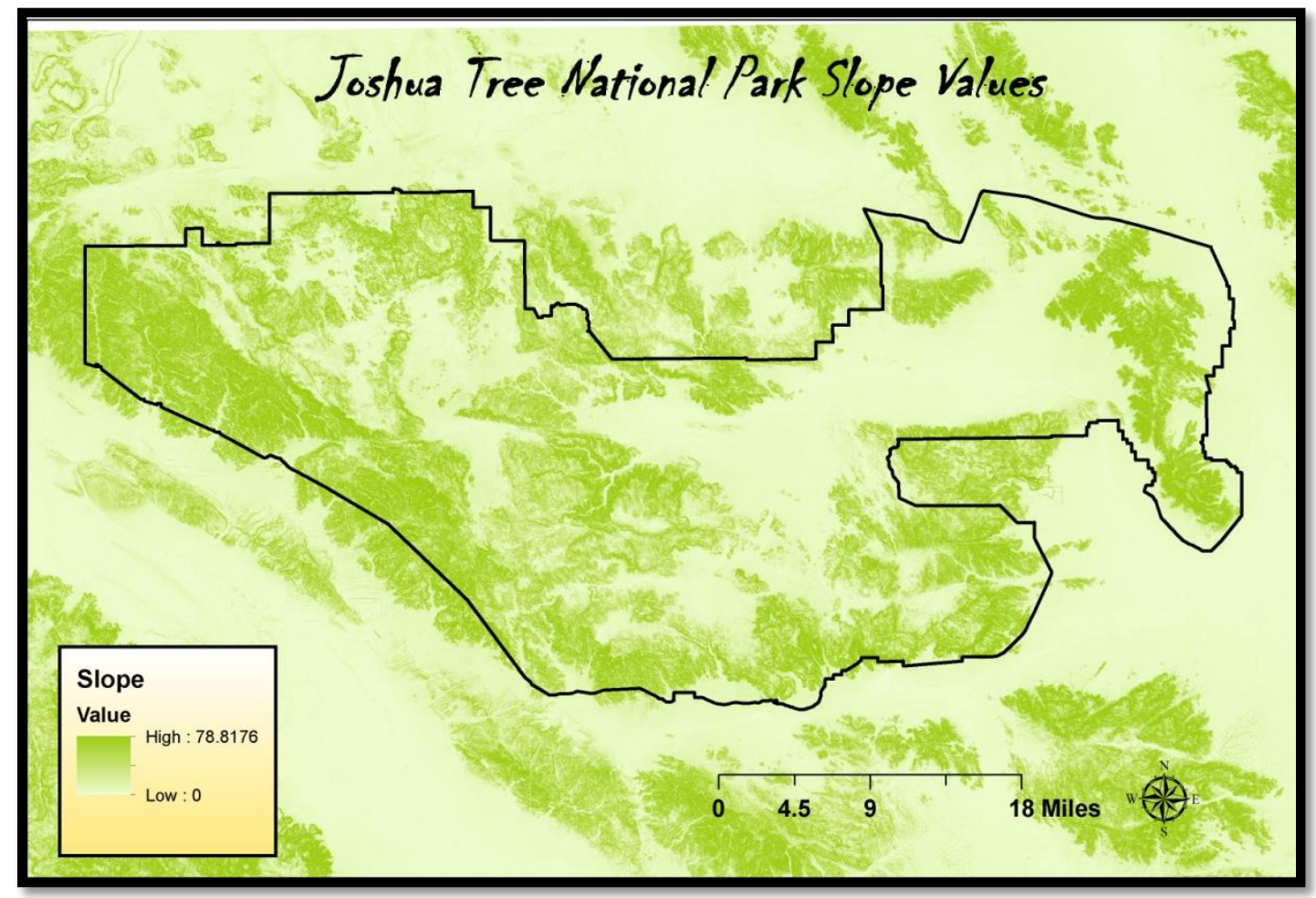

Figure 5-11 Slope Values

The output measurement determines the measurement units of the output slope data; this unit can be degree or percentage, and DEGREE was chosen in this project. In addition, if the $\mathrm{x}, \mathrm{y}$, and $\mathrm{z}$ units are in the same measurement units, then the $\mathrm{z}$-factor will equal one, which was the case for this project. However, if these units are different, then the z-factor must set to the appropriated factor, or the result will be incorrect (ESRI, ArcGIS 9.3.1 Help Document). The Single Output Map Algebra function then used the slope to enhance the speed of the no-data cells.

Table 5-4 shows the Map Algebra expression that means if the cell value in RoadsTrails equals 2 , then divide the speed limit 2 by (1+ slope); otherwise, leave it as the value of RoadsTrails raster dataset. The development team divided the speed limit 2 by $(1+$ slope $)$, because if the slope is zero then the speed will be 2 . In addition, if the slope is less than 1 , then when the speed limit 2 is divided by slope then the result will be greater than $2(2.0 / 0.3=6.67)$. To avoid these errors, the development team divided the 
RoadsTrails by $1+$ slope. For example, where slope is 1 , then the speed will be $1(2.0 /$ $(1+1)=1)$. In areas of higher slope, like 20 degree, the speed will be much lower $(2.0 /$ $(1+20)=0.095)$.

Table 5-4 Map Algebra Functions

\begin{tabular}{|c|c|}
\hline Function & Scripting Code \\
\hline SingleOutputMapAlgebra & $\begin{array}{l}\text { gp.SingleOutputMapAlgebra_sa("Con((C:IldatallFinal } \\
\text { MIPIIMIP.sdellMIP.DBO.ROADSTRAILS) EQ 2, (2.0 / (1 + } \\
\text { (C:IldatalIFinal MIPIIMIP.sdellMIP.DBO.SLOPE))), } \\
\text { C:IldatalIFinal MIPIIMIP.sdellMIP.DBO.ROADSTRAILS)", } \\
\text { finalData+ "IIMIP.sdelI TravelSpeed ", "'" + finalData+ } \\
\text { "IIMIP.sdellMIP.DBO.RoadsTrails';'" + finalData+ } \\
\text { "IIMIP.sdellMIP.DBO.Slope'") }\end{array}$ \\
\hline SingleOutputMapAlgebra & $\begin{array}{l}\text { gp.SingleOutputMapAlgebra_sa("(1.0 / ((C: :IdatallFinal } \\
\text { MIPIIMIP.sdelITravelSpeed )* 26.82))", finalData+ } \\
\text { "IIMIP.sdelITravelCost", "'" + finalData+ } \\
\text { "IIMIP.sdellTravelSpeed '") }\end{array}$ \\
\hline
\end{tabular}

The last step in creating the Travel Cost surface is to convert the speed limit values (0.0250845 to $50 \mathrm{MPH})$ into time (minutes). Because of the Weighted Distance tools, it is harder to travel through cells with high cost values than those with low cost values, and the speed will not be suitable as a travel cost measure. That is, the Cost Distance tool would consider it harder to travel on a road with a speed of $50 \mathrm{MPH}$ than on a road with a speed of $3 \mathrm{MPH}$, because 50 is a bigger number than 3 . Therefore, the development team decided to convert mile-per-hour speed into minutes-per-meter travel times by multiplying the speed by a conversion constant that converted miles-per-hour into meter-per-minutes, and then inversed that to get minutes per meter as seen in the equation below.

\section{1 mile/1 hour=1609.344 meters $/ 60$ minute $=26.82$ meters $/ 1$ minute} Invert: $1 /$ (26.82 meters/1minute $)=1$ minute $/ 26.82$ meters

The development team used the Map Algebra scripting syntax to convert the speed to time and create the Cost Travel raster dataset (Table 5-4). The expression used in Map Algebra is (1.0/ (TravelSpeed * 26.82)), which mean one divided by the output of multiple TravelSpeed raster dataset values by 26.82 .

\subsubsection{Run the Cost Distance Tool}

The development team then used the Cost Distance tool to determine the shortest weighted distance or accumulated travel cost from each cell to the nearest source location. This tool applies distance in cost units, not in geographic units, and it requires both a source dataset and a cost raster as input. The input for this tool was the Travel Cost raster dataset that was calculated previous steps, with the Fire Stations feature class as a source dataset. There are two fire stations, so the Cost Surface scripting tool selected each fire station and generated the cost distance for each of those fire stations. The 
output of the Cost Distance tool was the Cost Distance raster dataset that identified the least accumulative cost for each cell to return to the closest source location. The Backlink direction raster dataset was another output of the Cost Distance tool, and provided a road map to identify the route to take from any cell on the least-cost path back to the source cell. These outputs were generated for each fire station. To use the Cost Distance tool, the Spatial Analyst extension must be present; therefore, the tool checked this extension when it ran (Code in Appendix A).

\subsection{Firebreak Preparing Data Scripting Tool}

This tool was used to prepare the firebreak data to be used as an input for the Firebreak Priority Analysis tool, which would run through the web application. The reason for dividing the Firebreak scripting tool into two tools was the performance of the web application. This tool was created using a Python scripting language in order to perform a sequence of algorithms. It required multiple inputs: Inhabited Structure feature classes, Cultural Sites feature classes, Habitats feature classes, Vegetation feature class, and the data path folder (Figure 5-12).

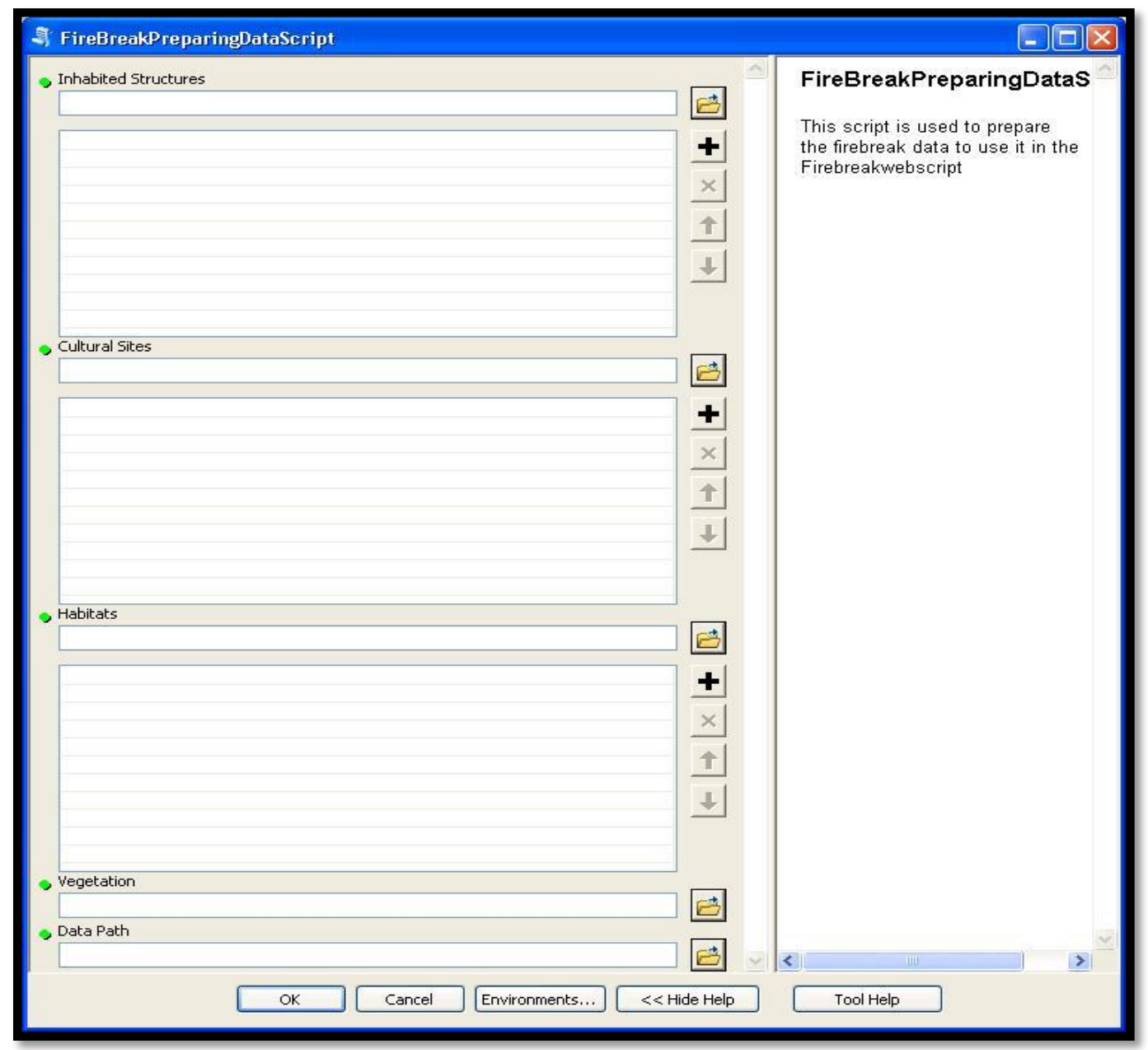

Figure 5-12 Firebreak Preparing Data Script Tool 
In Figure 5-13, the blue represents the processes, the orange represents the inputs feature classes, and the green represent the outputs feature classes. It also shows a personal-ArcSDE geodatabase to store the outputs of the processes and a folder to assign where the feature classes will be stored.

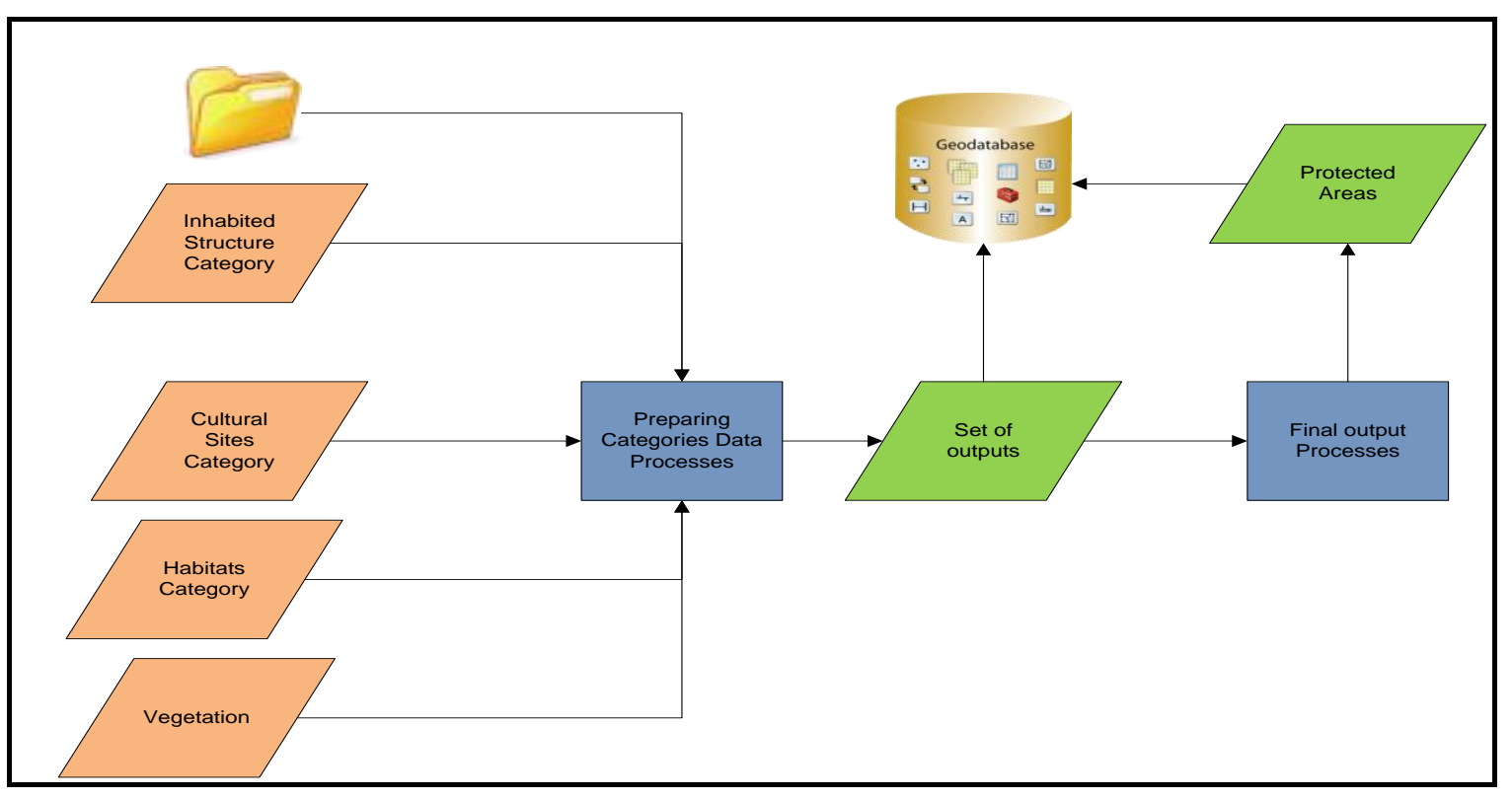

Figure 5-13 Firebreak Preparing Data Tool Process

The input categories for this tool were discussed with the client and advisor before the development team built this tool, which depended on the firebreak factors that the fire station staff considered most important to stop the fire. In addition, all the outputs from this tool would be stored in a personal-ArcSDE geodatabase. This section outlines the overall functions that the Firebreak Preparing Data scripting tool handled to prepare the final protected areas feature class. The result from this scripting tool will be used as an input for the Firebreak Priority Analysis tool (Figure 5-14). 


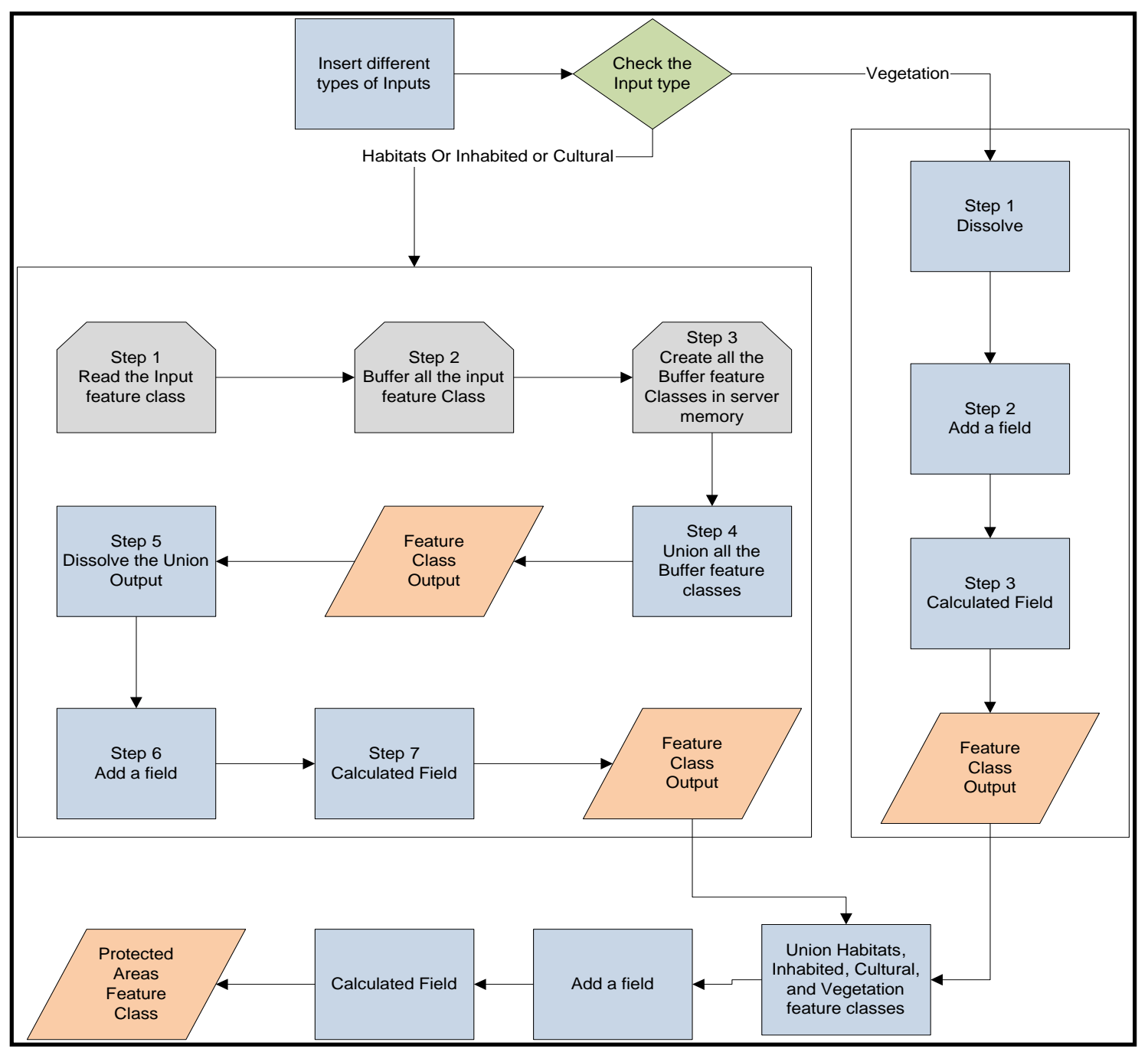

Figure 5-14 Overall Steps of Scripting Tool

Prior to development of this tool, many of the functions were tested, such as a Weighted Overly function, but these functions were not appropriate because the type of available data was not useful with this function. The Firebreak Preparing Data tool accepted various inputs categories including the Inhabited Structure category, Cultural Sites category, Habitats category, and Vegetation category. The tool read all the feature classes under each category and created a buffer polygon to a specified distance around each input feature. Most of the inputs feature classes type were point, which was the reason for using the buffer function. The buffer distance depended on the input category. The buffer distance around the inhabited structure and cultural sites categories were 25 meters while the buffer distance around the habited category was 5 meters. The tool created all the buffer feature classes in the server memory using the syntax "in_memorylfeatureName_Buffer". The tool then used the Union function to compute a geometric intersection of the input features, which was retrieved from the server memory. All features will be written to the output feature class with the attributes from the Input features, which it overlaps. 
The tool then used the Dissolve function to aggregate features based on specified attributes, in this case, which is the category type. A field was added to the Dissolve function output using the Add Field function, which is a function to add a field to the table of a feature class. The value of that field was calculated using the Calculate Field function and the value was dependent on the category type. All the above functions were repeated for Habitats, Inhabited Structures, and Cultural Sites categories.

The tool used the Dissolve function to aggregate the Vegetation feature class based on the vegetation category, and a field was added to the Dissolved function output. The value of that field was calculated using Calculate Field function, which depended on the vegetation category. After all inputs categories feature classes were prepared, the Union function was used again to compute a geometric intersection of all input features and created the Protected Areas feature class as an output. To manage the Protected-Areas types, the tool added a Final_Types field to the Protected Areas feature class to arrange the inputs categories types, and the values of that field calculated using Calculate Field function and a Python scripting function as below.

def Concat (*args) : // The function accept different arguments

$$
\text { retval }=" " \text { // An attribute to store the retrieve value }
$$

sep="_" // separate the types using " _"

for $t$ in args : // Loop to go through all the protected areas feature class

$\mathbf{s}=\mathbf{s t r}(\mathbf{t})$.strip() // Return a copy of the string with leading and trailing //characters removed

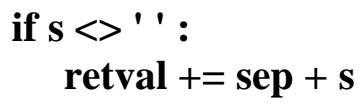

The last step in Firebreak Preparing Data Scripting tool is to concatenate the inputs categories under one protected area category type, using the Concat Python scripting function because these inputs were overlapped. Figure 5-15 shows the input before and after using the Concat Python scripting function.

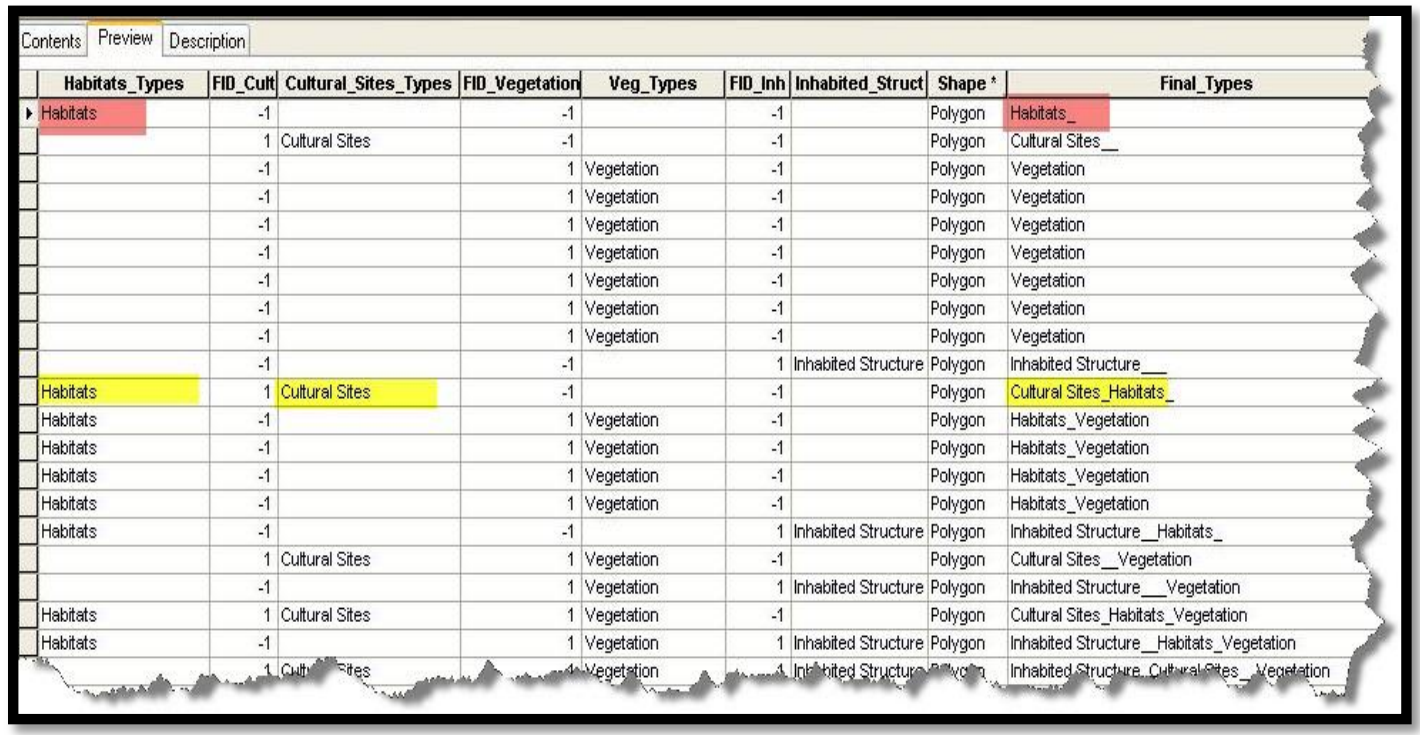

Figure 5-15 Concat Python Function Output 
The expression below was used to call the Concat function. (Appendix A) Concat(!Inhabited_Structure_Types!, !Cultural_Sites_Types!, !Habitats_Types!, !Veg_Types!)

\subsection{Other Python Scripting Tools}

There were two scripting tools prepared to run from the web application. These tools were created using a Python scripting language in order to perform a sequence of algorithms. To enhance the performance of the web application these tools were separated from the previous tools.

\subsubsection{Least Cost Path Scripting Tool}

The Least Cost Path scripting tool is one of the geoprocessing tools that was published as a web service to ArcGIS server 10 and is run by Flex API from the web application. It requires fire station used and incident location as inputs, and the output will be set automatically (Figure 5-16).

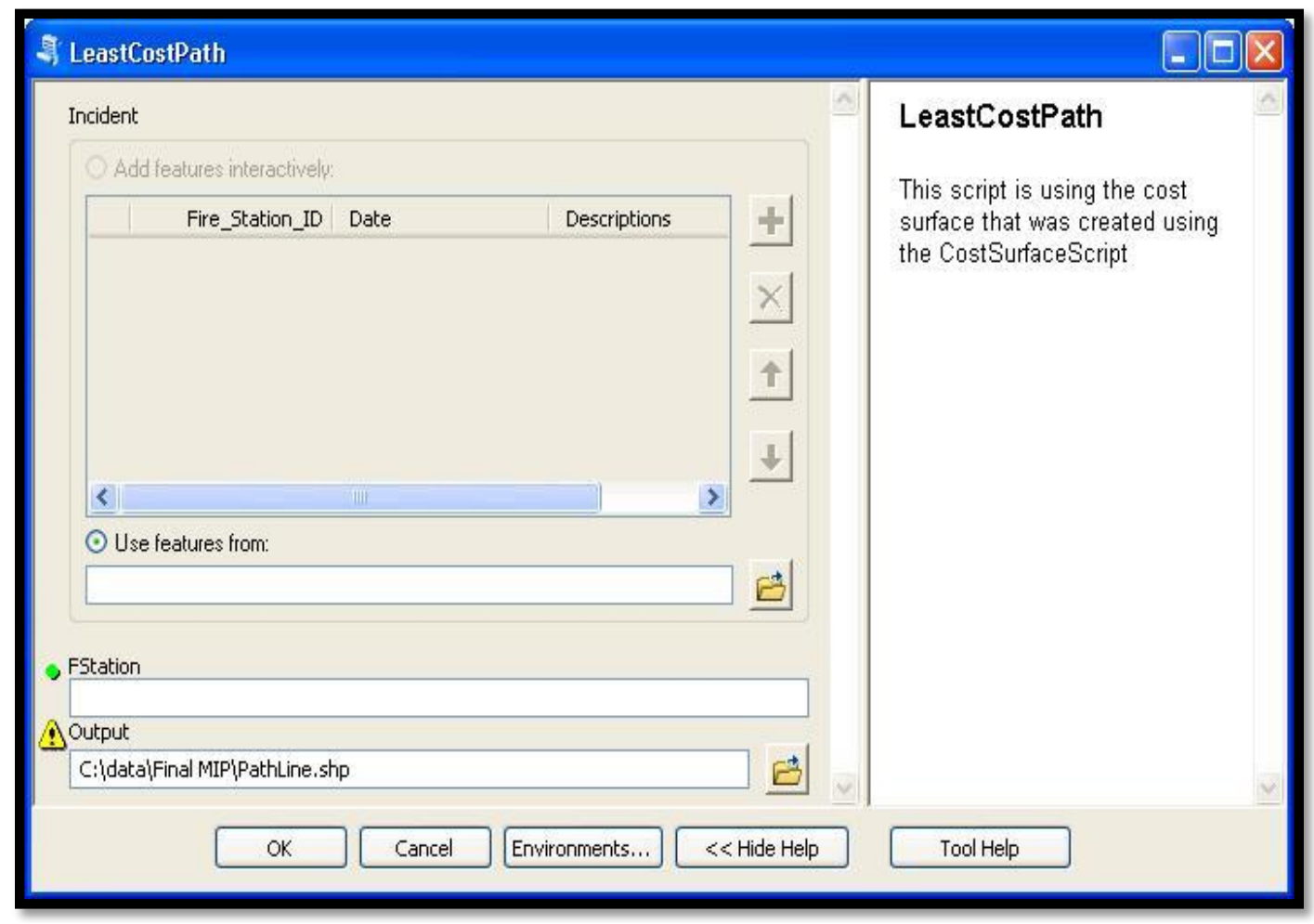

Figure 5-16 Least Cost Path Scripting tool

Figure 5-17 illustrates the overall functions that were used in this script. The tool accepted a fire station as a string input to determine which fire station was used to deal with fire incident, and acceptes a fire incident location as input. 


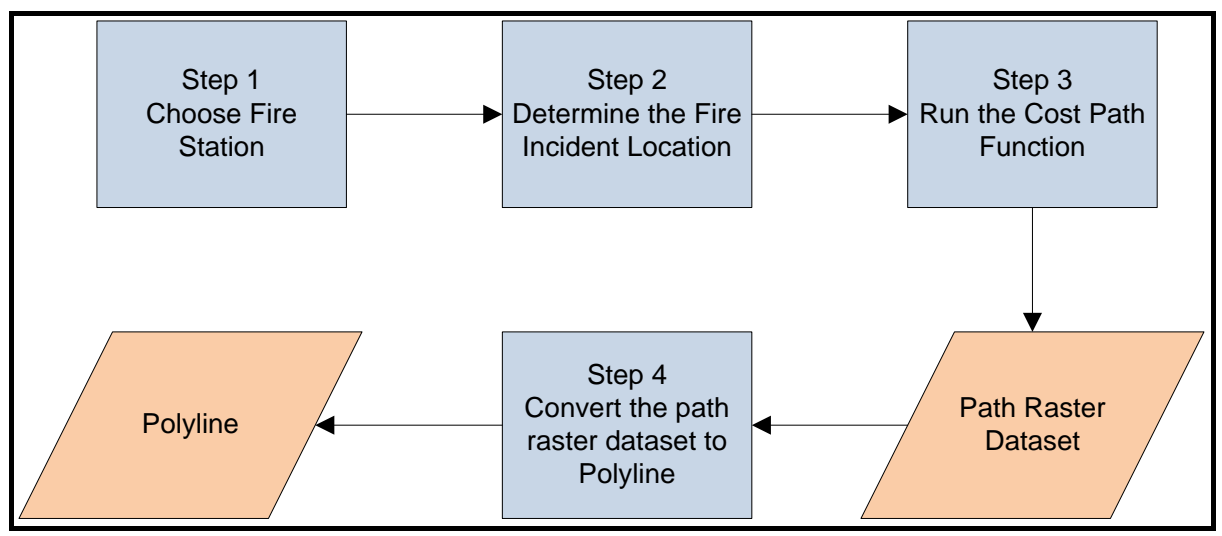

Figure 5-17 Overall Function in Least Cost Path Tool

Then, the tool will run the Cost Path function that depended on the fire station to calculate the least-cost path to the fire from a fire station. This function used the cost distance and backlink raster datasets that were prepared before for each fire station as inputs. This tool creates a least-cost path raster dataset from a fire station to the incident location. In addition, the tool converted the least-cost path raster dataset to polyline shapefile and stored it in the geodatabase folder to display it in the web application. It converted to vector to enhance the performance of the web application (Code in an Appendix A).

\subsubsection{Firebreak Priority Analysis Scripting Tool}

The Firebreak Priority Analysis scripting tool is another geoprocessing tool that published as a web service to ArcGIS server 10 and using Flex API to run this script from the web application. It requires an incident location, buffer distance as an input, and the output will be set automatically (Figure 5-18). 


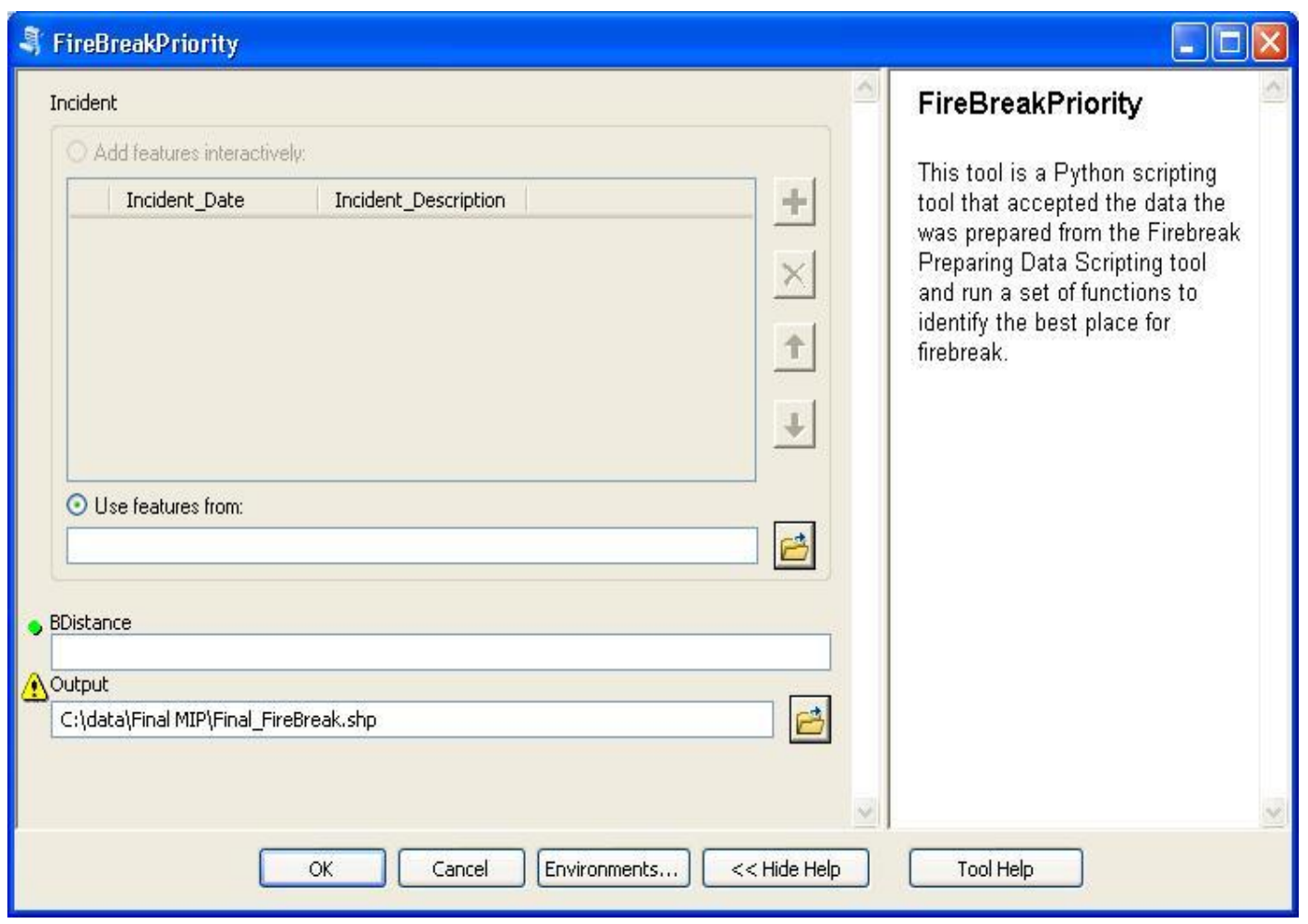

\section{Figure 5-18 Firebreak Priority Analysis Scripting Tool}

Figure 5-19 illustrates the overall functions that were used in this script. This tool acceptes a fire incident location as input to identify the best location for a firebreak before the fire impacted the protected areas.

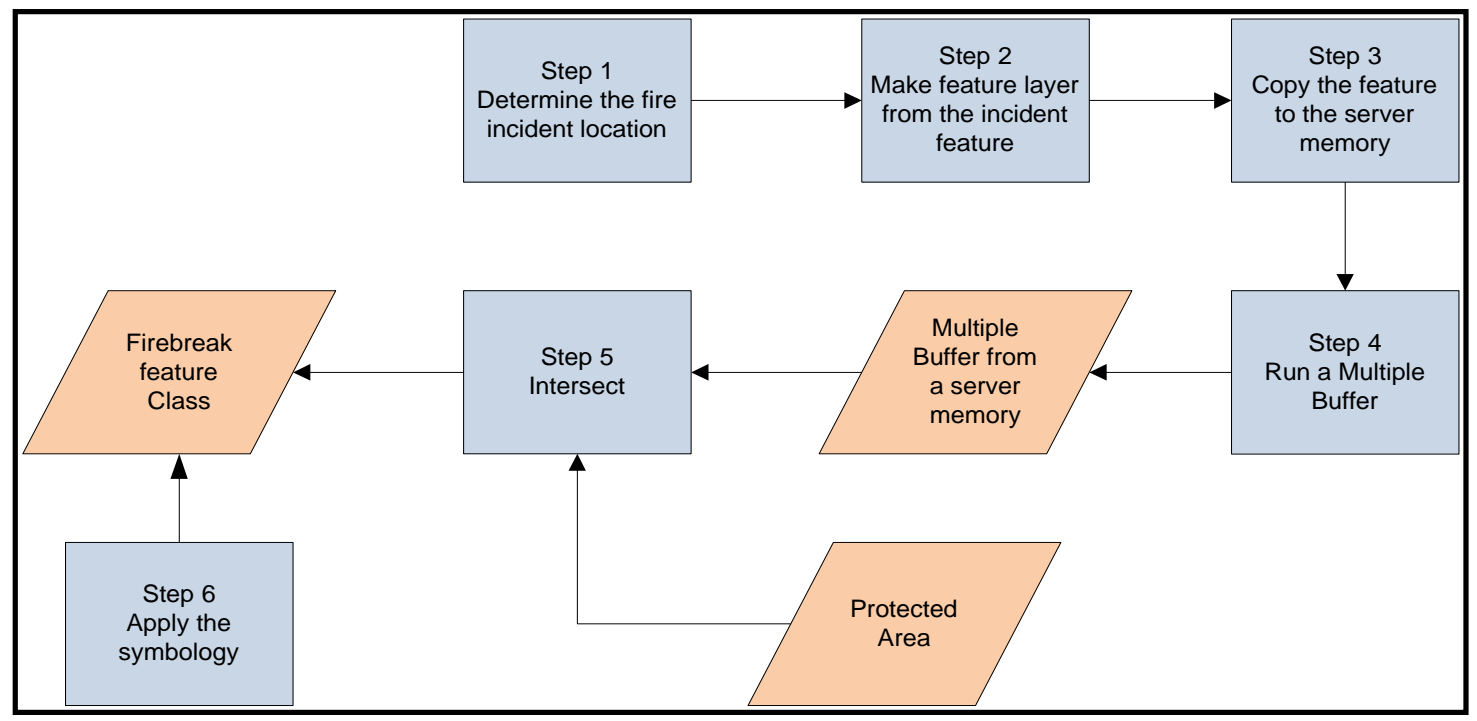

Figure 5-19 Overall Functions for Firebreak Priority Tool 
Then, the tool will run the Make Feature Layer Function to create a feature layer from the incident location input. The layer that was created by this function is temporary and it will not persist after the fire incident ends. The tool will copy the layer to a new feature class that created in the server memory using the Copy Feature function. The tool then will run a Multiple Ring Buffer function around the fire incident location and the output will save in the server memory. The tool will run the Intersect function that computes a geometric intersection of the Input features. The Input features of this function will be a multiple buffer feature and protected areas feature class. The output will be saved in the geodatabase folder for display the web application. The tool will use the Python SetParameterAsText syntax to apply the symbology for the output. (Code in an Appendix A)

\subsection{Web Application}

The last major component of this project was to create a web application. This web application was created using ArcGIS API for Flex. Prior to the Flex API web application, the development team had experience with different ArcGIS API. From this experience, the Flex API was found to be more interactive and it allows different functionalities on the web application. Moreover, Flex API enables the development team to create interactive and expressive web applications, leveraging ArcGIS server resources such as maps, locators, feature services, and geoprocessing tools and Flex component such as grids and charts.

In order to complete this task, the desired map service was first published to the ArcGIS server. Three major functions included locating the fire incident location using different search types, running a geoprocessing tool to identify the least cost path from fire station to the incident, and running a geoprocessing tool to identify the best place for firebreak.

\subsubsection{Publishing a Map Service Using ArcCatalog}

A map document that contained the basic layers such as the fire stations, and roads was first created in ArcMap (Figure 5-20). 


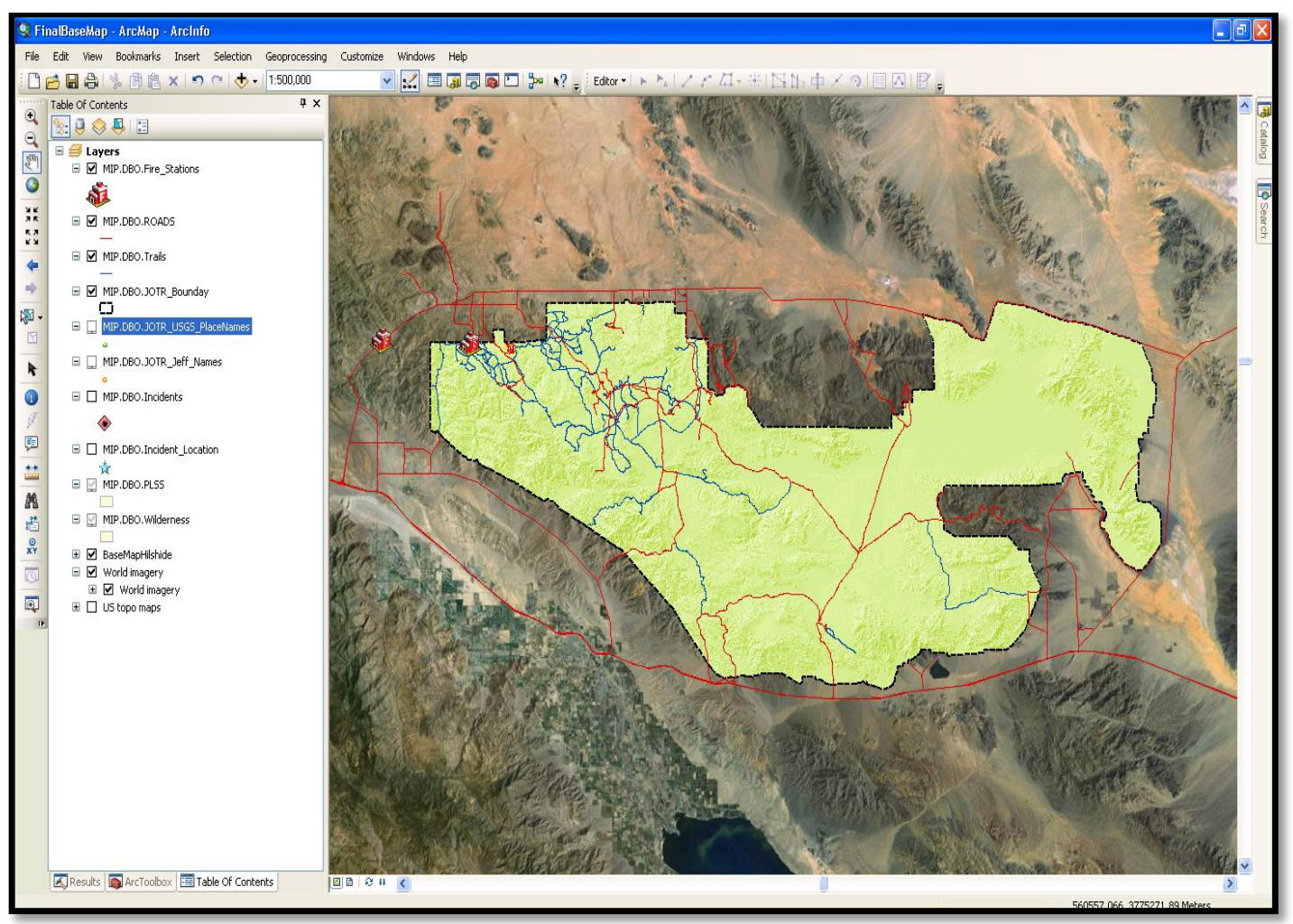

\section{Figure 5-20 FEMS BaseMap Document Overview}

Prior to creating the ArcMap document, the coordinate system of the data frame was set to NAD_1983_UTM_Zone_11N because it was the projection required by the client. The development team used the scale range level to show some layers at a specific zooming level, which keeps the map from getting crowded, and helps the user to understand the map better. This map document was then published to an ArcGIS Sever by using the Add GIS Services wizard in ArcCatalog. Before publishing the FEMSBaseMap web service, the development team chose the Feature Access option to make the web service editable (Figure 5-21). 


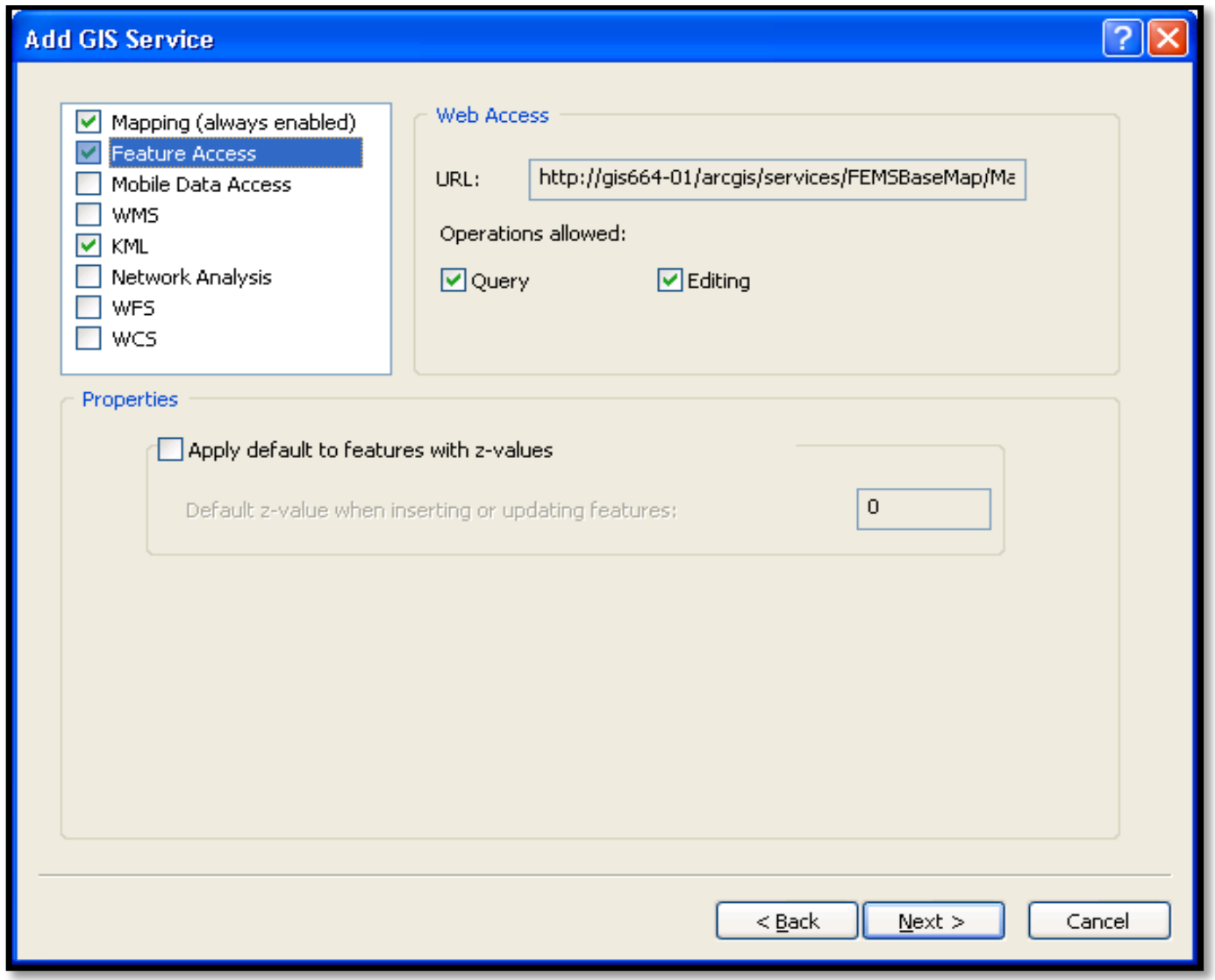

Figure 5-21 Map Document Editing Option

Once the publishing task was completed, the map document was added to a list of map services in the ArcGIS Services Directory. The spatial reference was "26911" and refers to the NAD_1983_UTM_Zone_11N coordinate system, and unique IDs for each layer. For example, " 0 " and " 1 " IDs were assigned to the fire station and roads layers, respectively (Figure 5-22). 


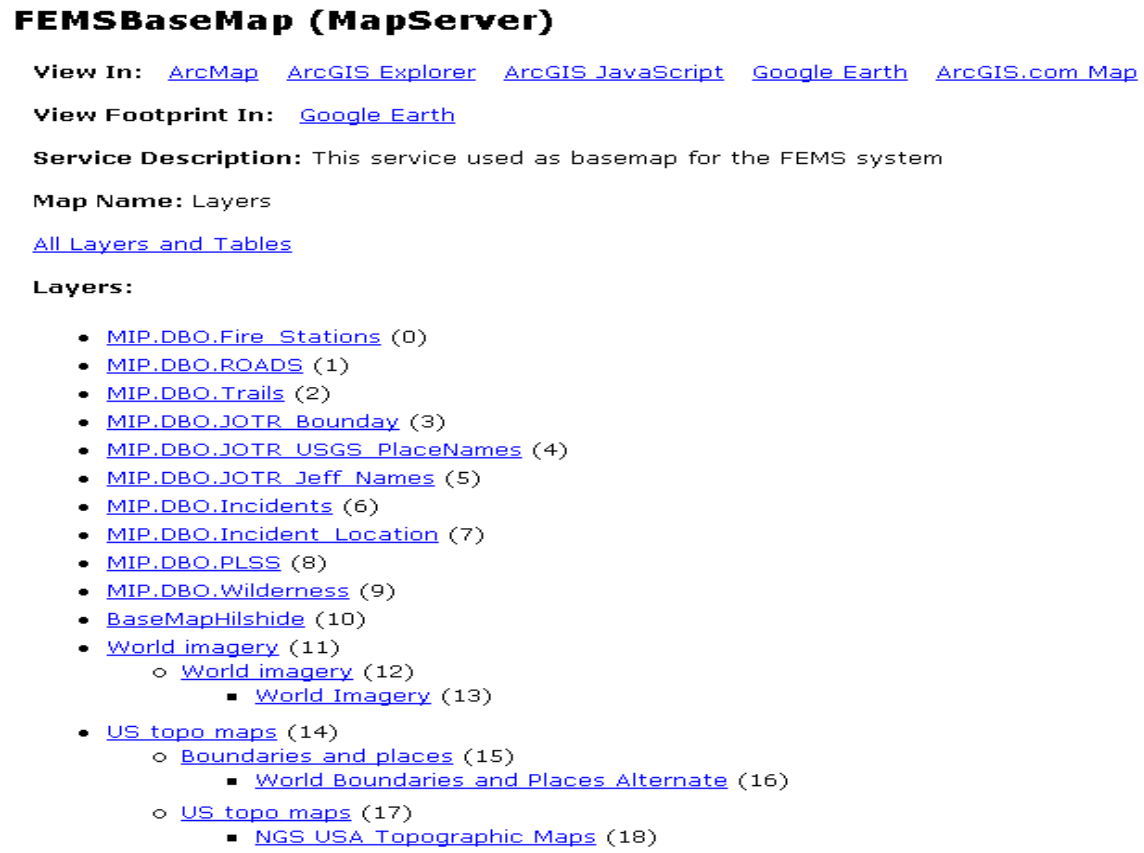

Figure 5-22 FEMS BaseMap Web Service

After publishing the FEMSBaseMap ArcMap document to an ArcGIS Server as a web service, another web service was created which related to the feature Access option during the service publishing. This service exposed access to vector feature geometries and attributes and is a new feature with ArcGIS Server 10. A great benefit of feature services is the ability to expose feature editing through Flex application. In addition, feature services are published from map documents, and the source datasets are required to reside in a single ArcSDE geodatabase (Figure 5-23).

\section{FEMSBasemap (Featureserver)}

View In: ArcGIS.com Map

Service Description: This service used as basemap for the FEMS system Layers:

- MIP.DBO.Fire Stations (a)

- MIP.DBO.ROADS (1)

- MIP.DBO.Trails (2)

- Mip.dBo.jotr Bounday (3)

- Mip.dBo.jotr usgs placenames (4)

- MIP.DBo.jotr jeff Names (5)

- MIP.DBO.Incidents (6)

- MIP.DBO.Incident Lacation (7)

- MIP.DBO.PLSS (B)

- MIP.DBO.Wilderness (9)

Tables:

supported Interfaces: REST SOAP

Figure 5-23 Feature Web Service 


\subsubsection{Developing Geoprocessing Services and a Geometry Service}

The two Python geoprocessing tools discussed in section 5.3 were developed to run in the web application. These tools were necessary in order to minimize the required time for displaying the analysis results in the web application. The tools were published as a geoprocessing services by using the Add GIS Services wizard in ArcCatalog. An execution type of this geoprocessing service was changed to "Synchronous" in the Add GIS Services wizard (Figures 5-24 and 5-25).

CMPWebToolbox (GPServer) - Windows Internet Explorer
Mome > MIPWebToolbox (GPServer)
MIPWebToolbox (GPServer)
Service Description: This is a geoprocessing tools that were published as a geprocessing services and used to run from the web application
Tasks:
• FireBreakPriority
Execution Type: esriExecutionTypeSynchronous
Result Map Server Name:
Supported Interfaces: REST SOAP

\section{Figure 5-24 Geoprocessing Services}




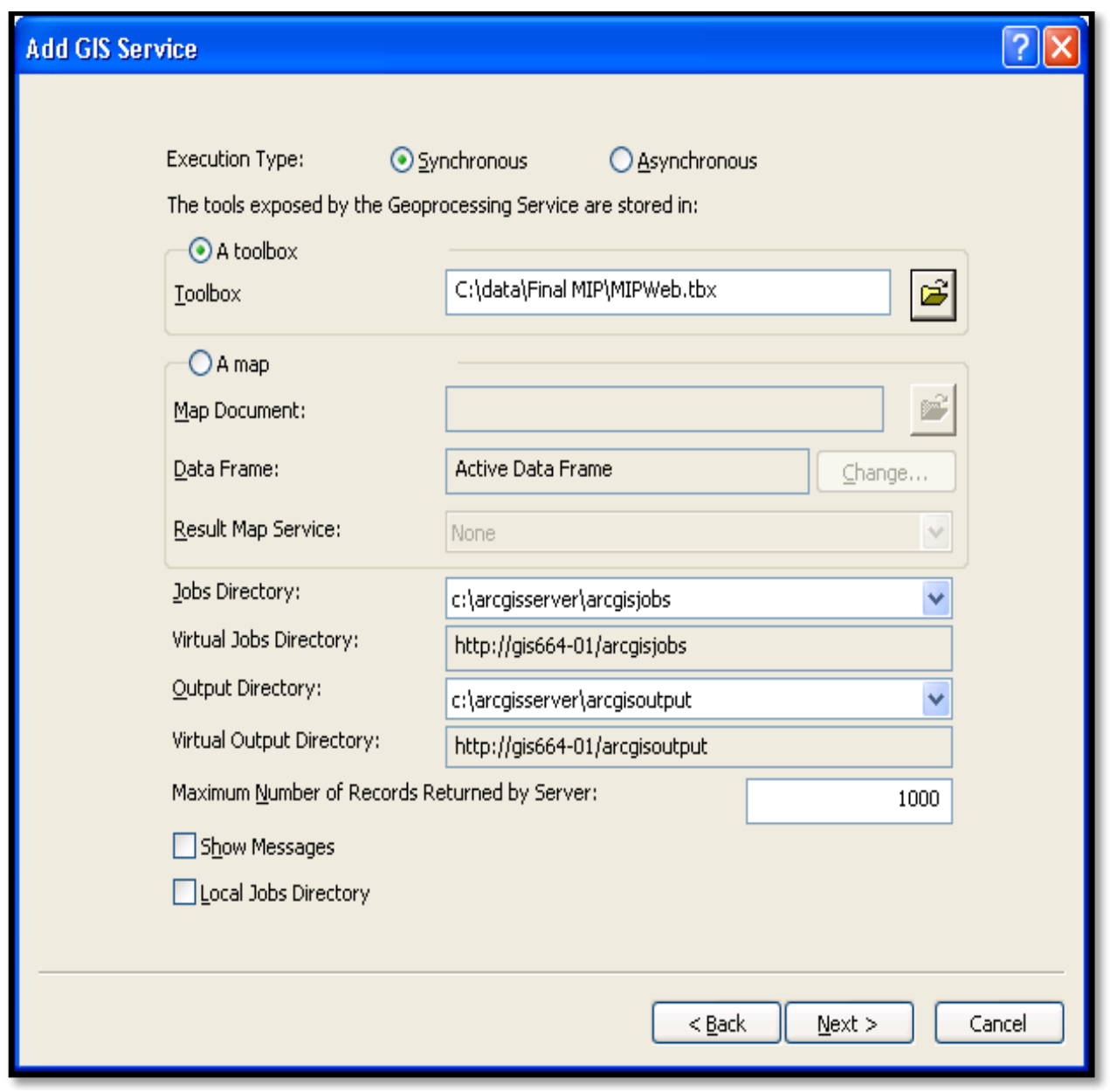

\section{Figure 5-25 Selecting the Scripting Toolbox and the Execution Type}

This type of execution meat the computer will wait while the task executes on the server, while the execution type "Asynchronous" means the computer does not have to wait for the tool to finish execution and the user can move on the other tasks. Therefore, the development team decided to choose the "Synchronous" execution type because it is best suited for quick processes and they wanted the user to wait until the result set jobs executed. The results of these tools were saved in geodatabase folder each time the tool's previous results were overwritten.

The development team developed a Geometry service that will help applications to do geometric calculations, such calculating areas and lengths (Figure 5-26). This type of service is only visible to server administrators and developers. 
ArcGIS Services Directory

Home $>$ Geometry (GeometryServer)

Help I API Reference

\section{Geometry (GeometryServer)}

Service Description:

Supported Interfaces: $\underline{\text { REST }}$ SOAP

Supported Operations: Project Simplify Buffer Areas And Lengths Lengths Label Points Relation Densify Distance Union Intersect Difference Cut TrimExtend offset Generalize Autocomplete

Reshape ConvexHull

\section{Figure 5-26 Geometry Service}

\subsubsection{Developing a Web Application Using Flex API}

Once the Python scripting tools were published as a geoprocessing service to the ArcGIS server, as well as the FEMSBaseMap published as a web service, a new web application was created using the Flex API. Figure 5-27 shows the workflow process of the web application, in which blue represent the processes, orange represents the manual inputs, green represents the display-outputs, purple represents the decision process, and yellow represents the drawing and navigation tools inputs. It also shows a personal-ArcSDE geodatabase to store and update the fire incident location, and gray represents the printed map output. 


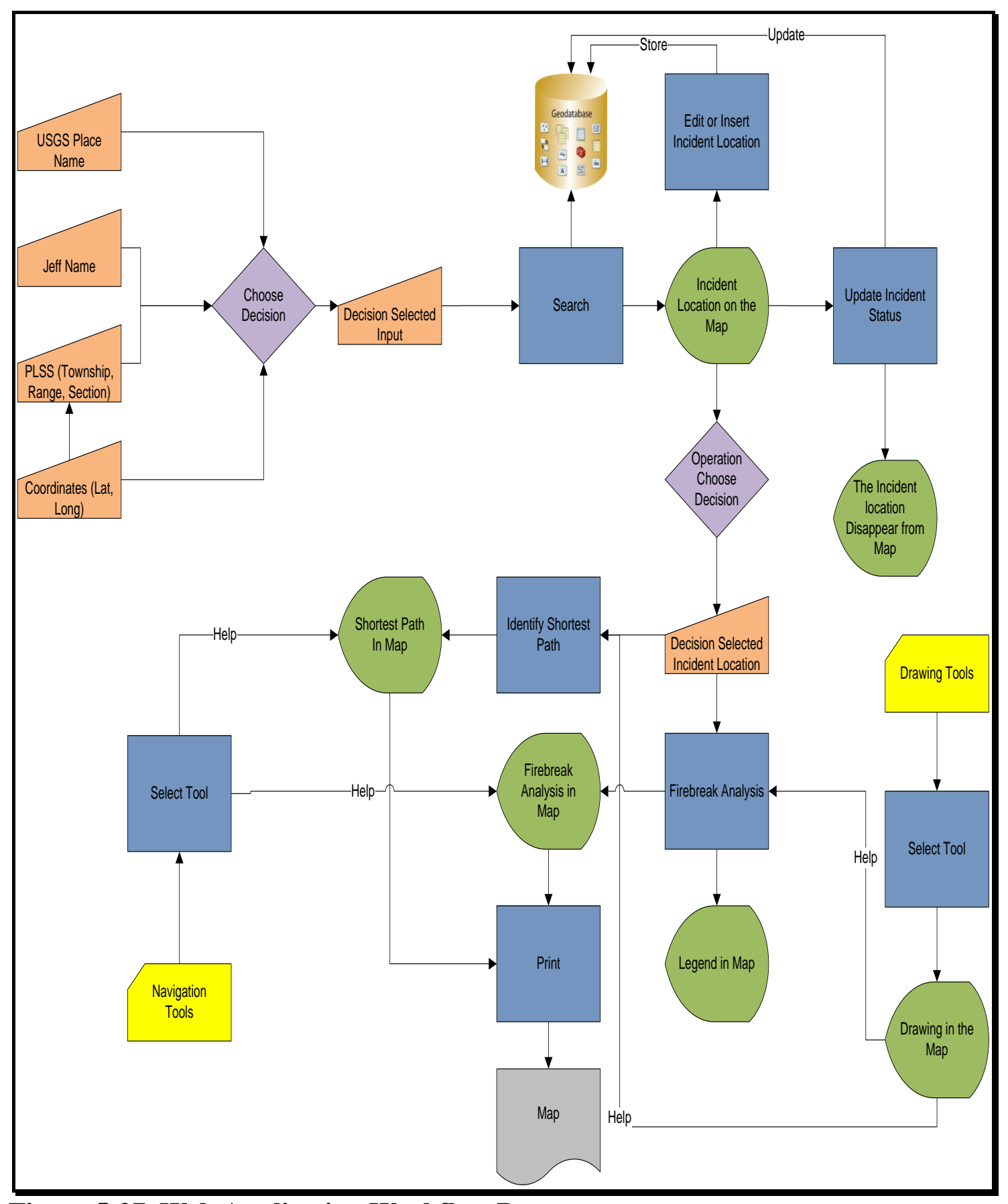

Figure 5-27 Web Application Workflow Process

The web application was designed to meet the client requirements, including identifying the fire incident location using different search types, identifying the least cost path from fire station to the incident, and identifying the best place for firebreaks. Therefore, the Querying task in Flex API was used to query information from an ArcGIS server service using the layer ID and display the results in the web application. For example, the development team retrieved information from the JOTR_USGS_PlaceNames layer by setting the query task uniform resource locator 
(URL) to the layer ID of " 4 " and executing the Query using the Query Parameters. The results are displayed in the web application. There were four different types of search that the web application could do: searching by JOTR_USGS_PlaceName, searching by JOTR local names (Jeff names), searching by PLSS, and searching by coordinates system (Figure 5-28). All of these types used the Flex API querying task to retrieve the data from the layers.

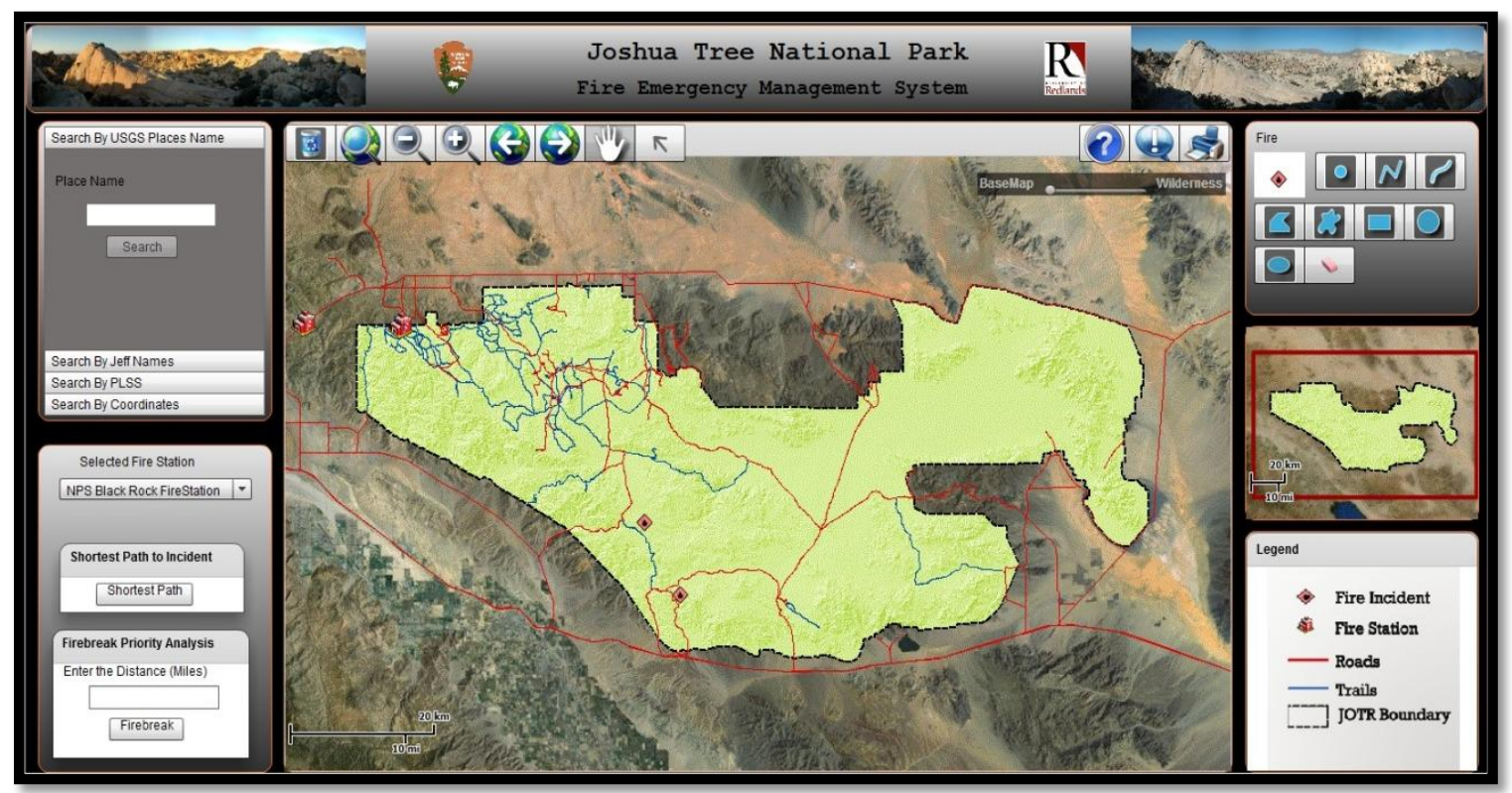

Figure 5-28 Web Application Different Types of Search

The web application allows editing features, which will edit the Incident feature class and store the incident location in this feature class. The web application will also run a geoprocessing service to identify the least-cost path from the fire station to the incident location. The web application will run a Firebreak geoprocessing service and display the result of the service in the web application. A legend will be added to the web application when the result of the Firebreak geoprocessing tool is displayed in the application. The web application showed the coordinates of the mouse pointer and allowed printing maps (Code in an Appendix B).

\subsection{Summary}

This chapter provided a detailed discussion of the various scripting methods used for the implementation of the project. It contains four major sections, including the scripting tool used for preparing the firebreaks data, the scripting tool used to prepare the cost surface, the geoprocessing scripting tool used to run from the web application, and the web application that was created using Flex API. Further discussion on the geoprocessing tools and web application results will be reviewed in Chapter 6. 


\section{Chapter 6 -Results and Analysis}

The first five chapters discussed system design and the procedures that were used to accomplish the main tasks requested by the client. This chapter presents major project results, and discusses the issues encountered during the implementation. Section 6.1 discusses the results of GIS tools developed to prepare the data for the geoprocessing tools inputs. Section 6.2 discusses the web application key functions that were implemented using ArcGIS Flex API, and the results of the geoprocessing tools developed to run from the web application.

\subsection{GIS Tools Installation and Results}

Four GIS tools were created using Python scripting programming language and ESRI ArcGIS 9.3.1 package with an ArcInfo License. These tools require the Spatial Analyst extension to be present.

Within an ArcMap or an ArcCatalog session, open the ArcToolbox Window. A right mouse-click button in the white area of this window will open a menu to select Add Toolbox option (Figure 6-1).
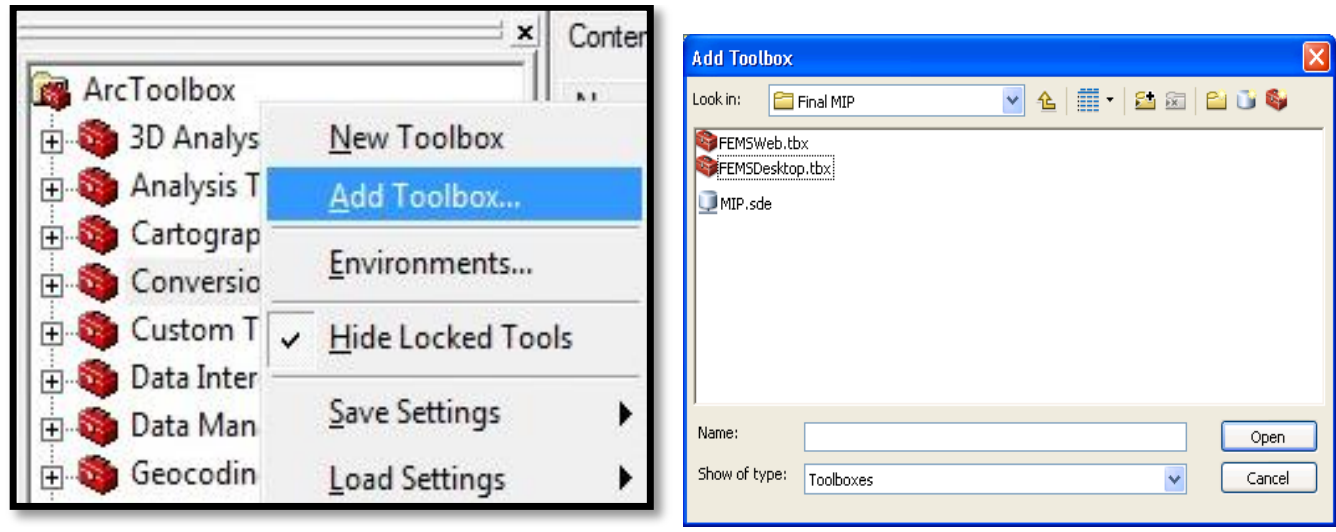

Figure 6-1 Adding the FEMSDesktop Toolbox

When the browser window opens, navigate to the folder and add the FEMSDesktop toolbox, which contains two Python scripting tools: the Cost Surface Scripting tool and the Firebreak Preparing Data Script. The toolbox is now added to the current session and the admin user can use these tools to prepare the data inputs for the other twogeoprocessing tools. In addition, there is another toolbox, called FEMSWeb, which is published as a geoprocessing service to run from the web application.

\subsubsection{Cost Surface Analysis Tool Results}

The Cost Surface Analysis Tool was dependent on the Roads, Trails, Fire Station feature classes and the Terrain raster dataset to produce the cost surface for each fire station. It also produces a set of raster datasets, such as the Elevation, RoadsTrails, Slope, and TravelCost, that are used to prepare the final output for that tool. These raster datasets 
were stored in a personal-ArcSDE geodatabase. Figure 6-2 shows the TravelSpeed raster dataset generated from this tool.

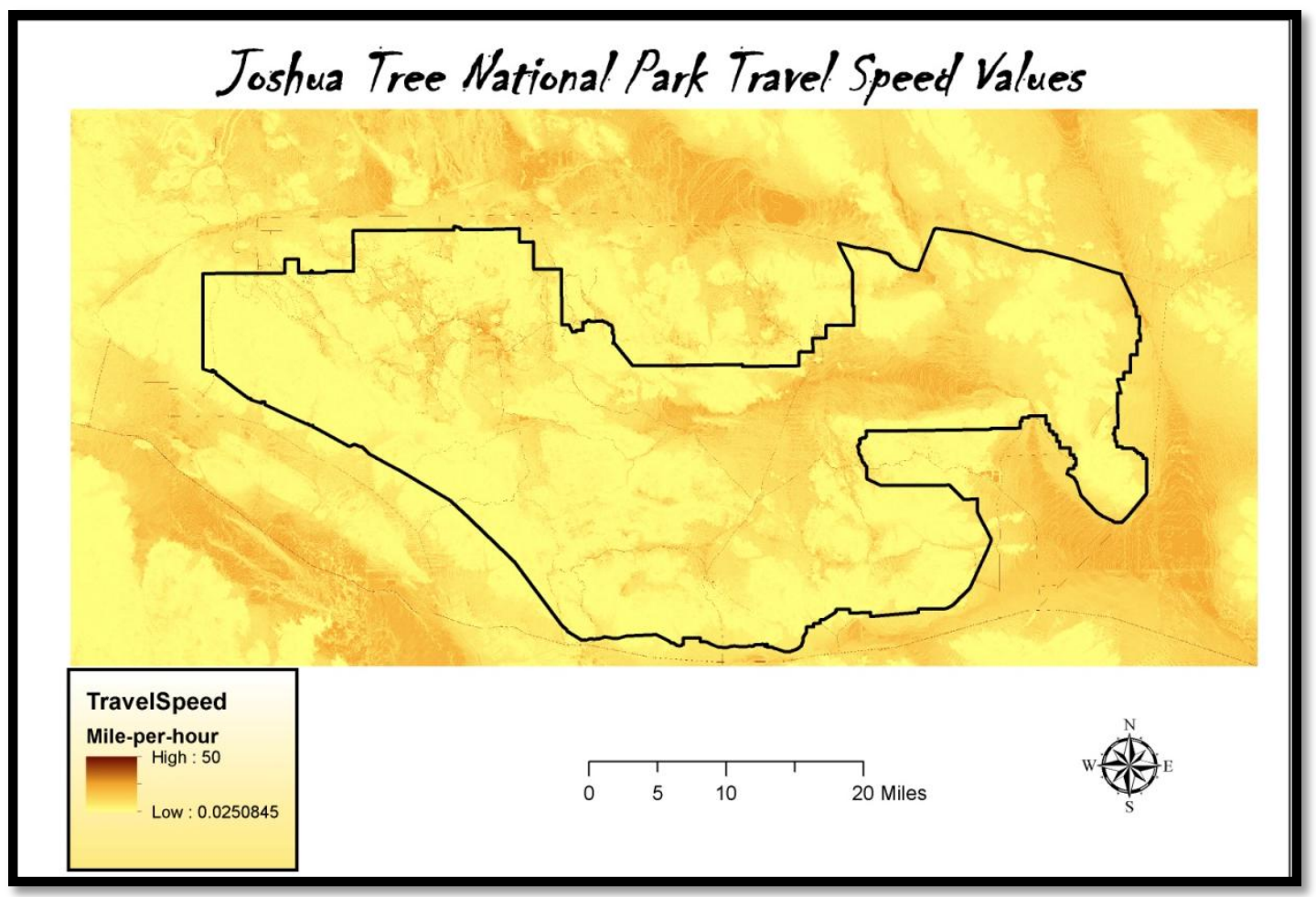

\section{Figure 6-2 TravelSpeed Raster Dataset Output}

This raster dataset shows the speed of the analysis surface after using Slope raster dataset. The benefit of using Slope raster dataset is enhancing the speed of the off-road area, which range from 0.0250845 to $50 \mathrm{MPH}$. Figure 6-3 shows the Travel Cost raster dataset after converting the speed into time. This output contains a speed value that ranges from 0.000745712 to 1.4864 minute-per- meter. 


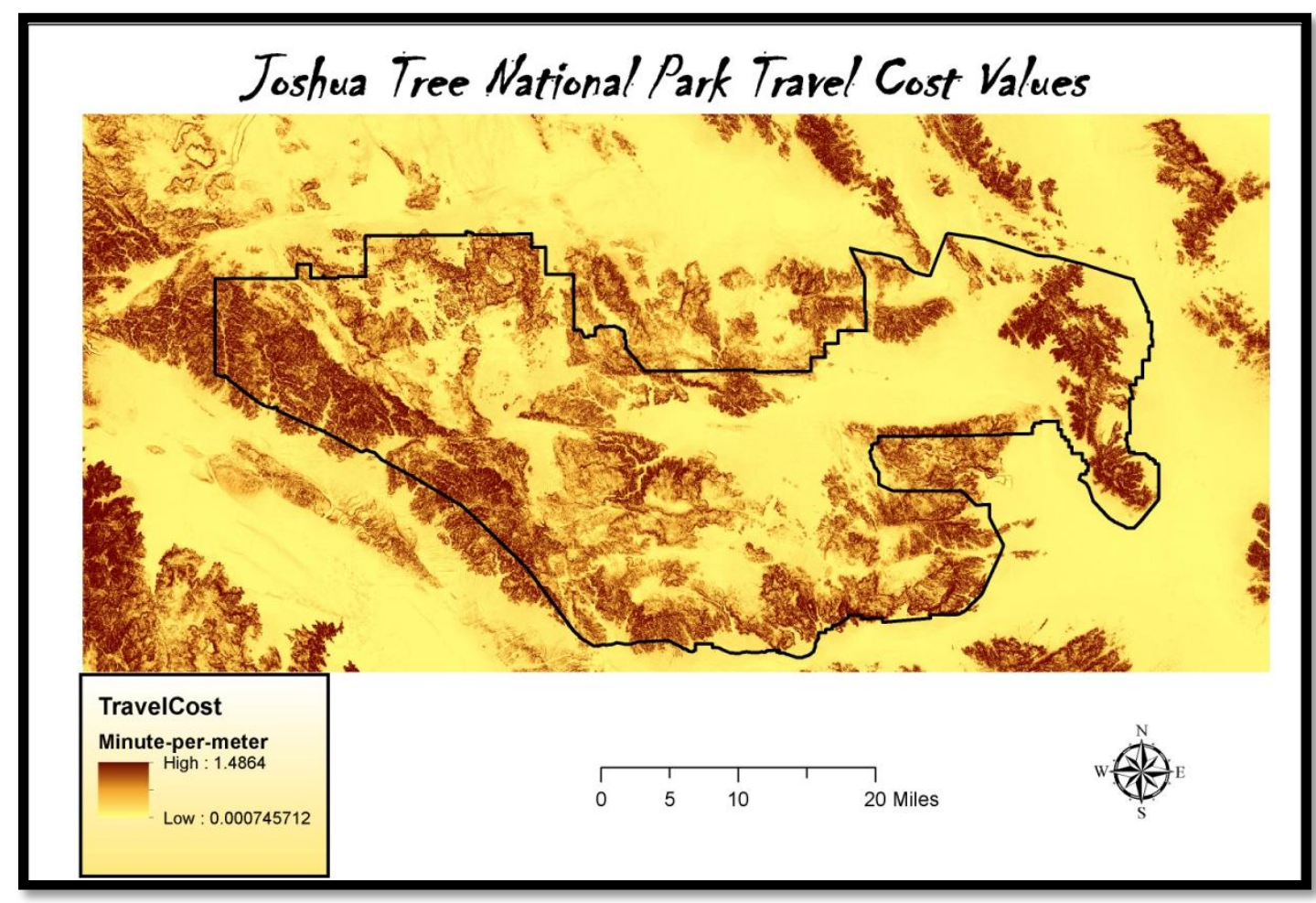

\section{Figure 6-3 Travel Cost Raster Dataset Output}

After the Travel Cost raster dataset was created, the Cost Surface Analysis tool uses this raster dataset to generate the Cost Distance and Backlink raster datasets for each fire station. Figure 6-4 shows the Cost Distance and Backlink raster datasets for NPS fire station. 

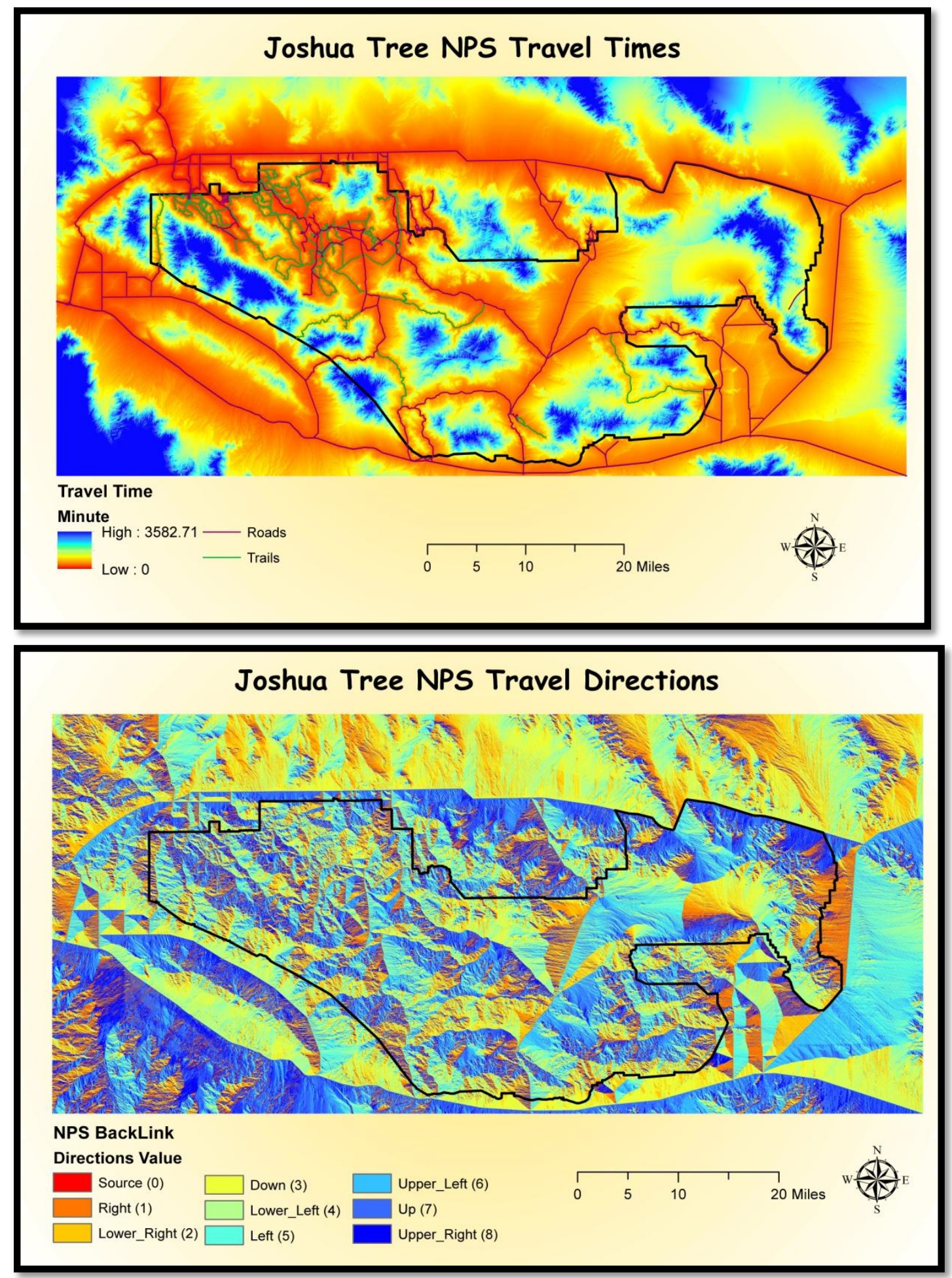

Figure 6-4 Cost Distance and Backlink Raster Datasets for NPS Fire Station

Figure 6-5 shows the Cost Distance and Backlink raster dataset for the BLM fire station. 

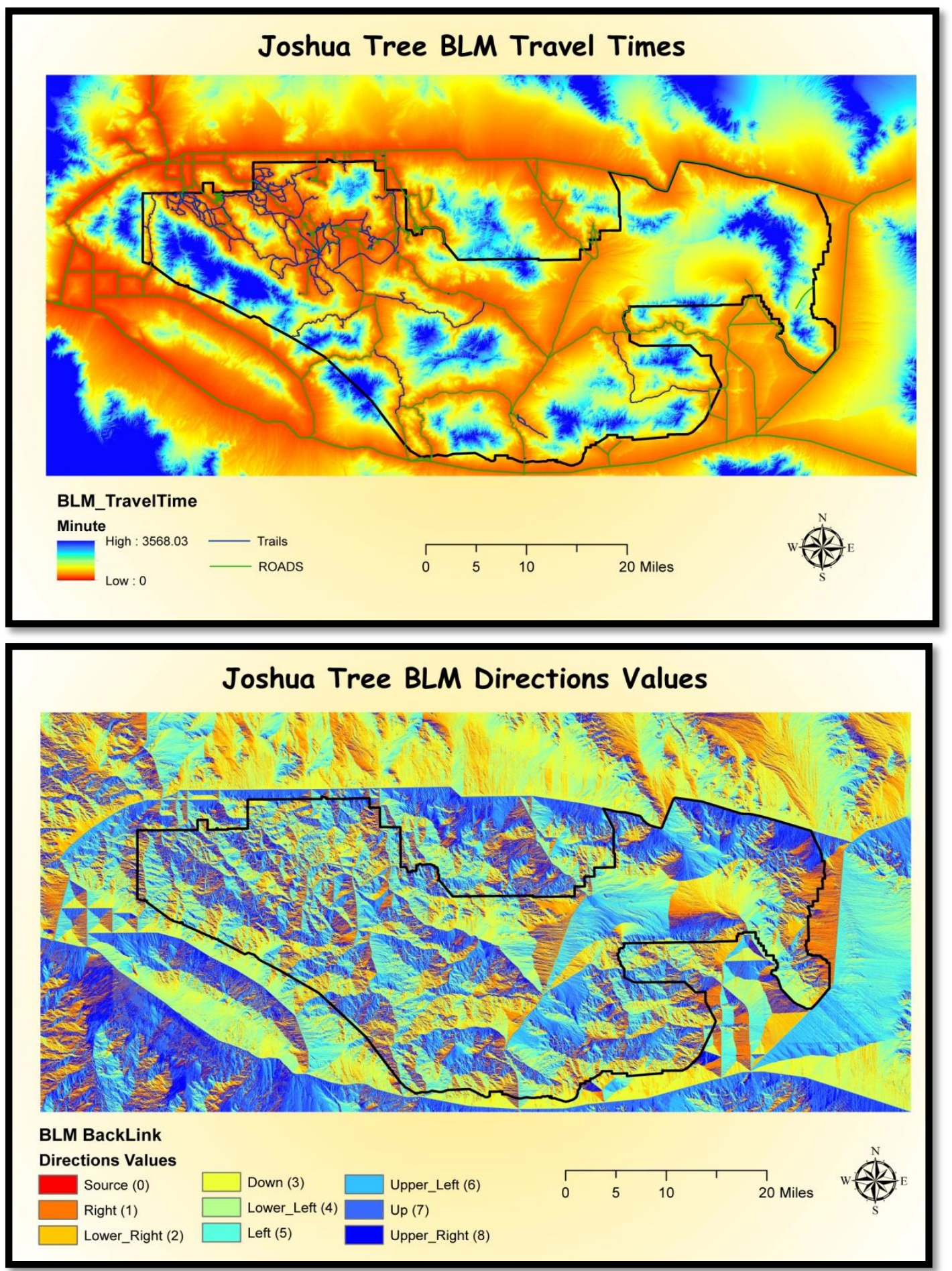

Figure 6-5 Cost Distance and Backlink Raster Datasets for BLM Fire Station

In Figures 6-4 and 6-5, the Cost Distance raster dataset, which is an output of the Cost Distance function, displays values ranging from low to high. This function is using to prepare a cost-weighted distance analysis. For example, the function starts from the BLM fire station, and then spreads to every other cell in the study area, accumulating 
their travel costs as it does so. Since the cost surface units are minutes per meter, the output will be the number of minutes needed to go from a BLM fire station to every cell in the study area. In Figure 6-5, the values ranging from 0 to 3568.03, represent the number of minutes it takes to drive or go from a BLM fire station to each cell. The Cost Distance raster dataset shows the travel times that increase quickly as the fire trucks move away from a road. The values in the Backlink raster identify, for every cell, the neighboring cell on the least-cost path back to the source cell.

\subsubsection{Firebreak Preparing Data Tool Results}

The Firebreak Preparing Data Tool was dependent on the multiple inputs that are divided to four categories: Inhabited Structure feature classes category, Cultural Sites feature classes category, Habitats feature classes category, and the Vegetation category. It was designed to produce the Protected Areas feature class that will be used in the Firebreak Priority Analysis tool, which would run through the web application. Figure 6-6 shows the primary inputs for this tool and a folder to assign where the feature classes will be stored.

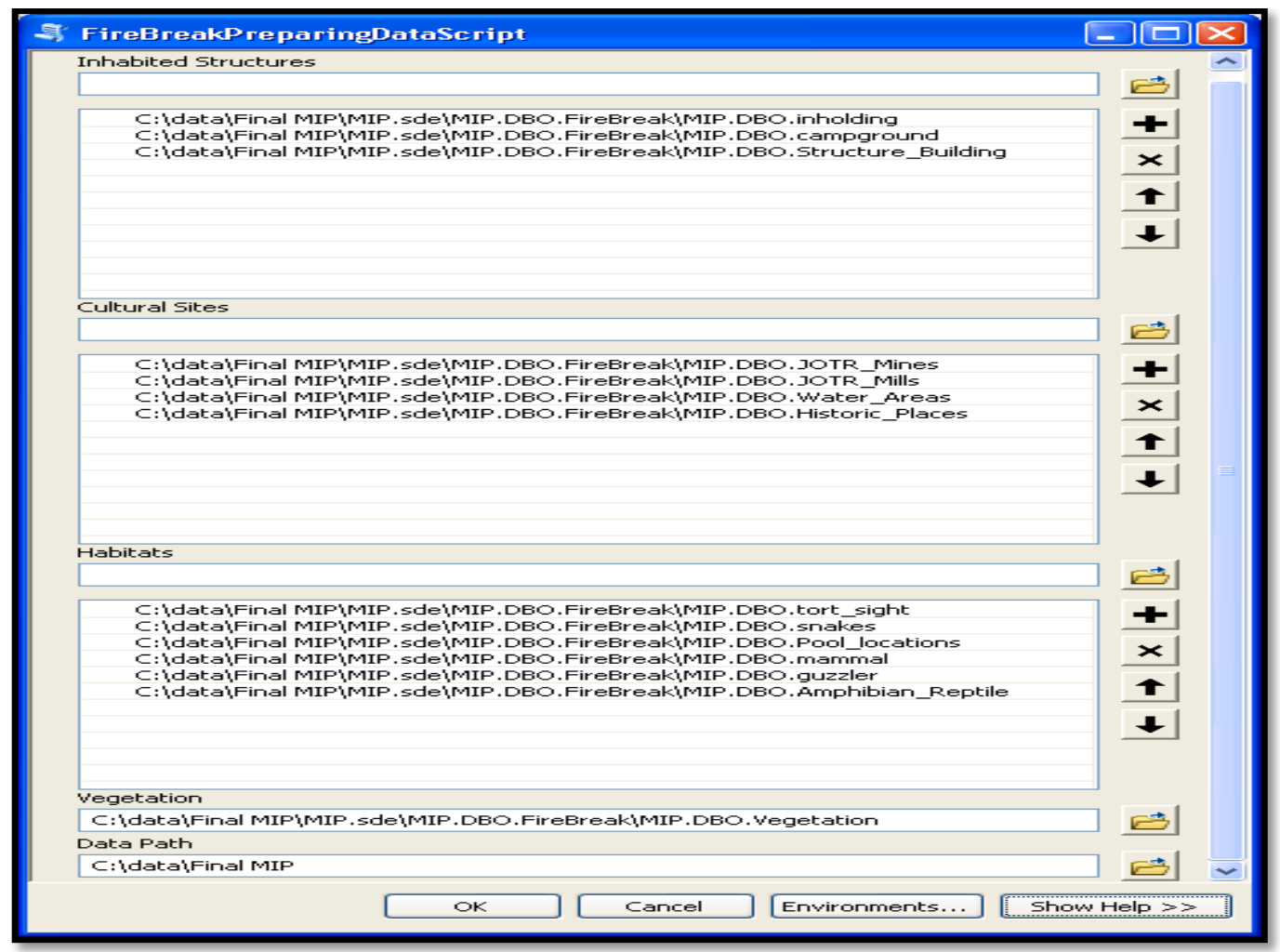

Figure 6-6 Firebreak Preparing Data Tool Primary Inputs

\subsubsection{The Tool Results}

The Firebreak Preparing Data tool produces a set of features class used to prepare the Protected Areas final output. These features classes were stored in a personal-ArcSDE 
geodatabase. Figure 6-7 shows the final output where the different types overlapped since the most of the data types were points.

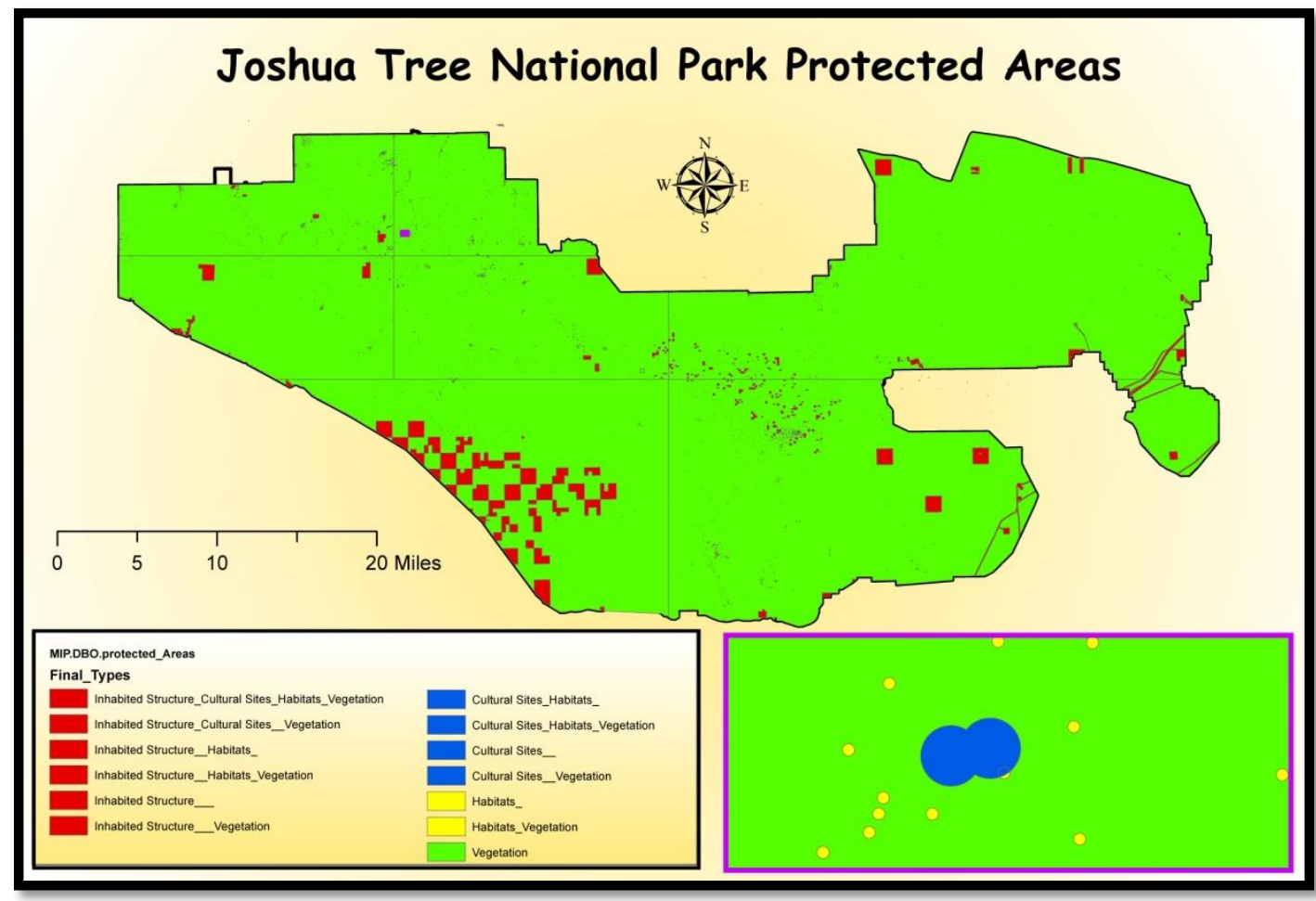

Table

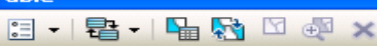
MIP.DBO.protected_Areas Shape *

Polygon

Habitats

intes

Cultural Sites

Vegetation

Vegetation

Vegetation

Vegetation

Vegetation

Vegetation

Inhabited Structure

Cultural Sites_Habitats

Habitats_Vegetation

Habitats_Vegetation

Habitats_Vegetation

Habitats_Vegetation

Inhabited Structure_Habitats

Cultural Sites Vegetation

Inhabited Structure _ Vegetation

Cultural Sites_Habitats_Vegetation

Inhabited Structure_Habitats_Vegetation

Inhabited Structure_Cultural Sites__Vegetation

Inhabited Structure_Cultural Sites_Habitats_Vegetation

Final_Types

Shape.area

4242.185294

26281.030979 474354392234559

753377420.216655

275474753.926507

317290393.82967

247758158.830076

877809041.756065 85882.730743 312.576446 5456.7557 64962.354549 105956.480616 15217.100083

78.141154

963168.442145

46310.442145

2664.883611

7368.558166

36299.876132

171.375822

MIP.DBO.protected_Areas

Figure 6-7 Protected Areas Features Class 


\subsection{FEMS Web Application Functions Results}

The FEMS web application using Flex API, is illustrated in Figure 6-8. It provides users with tools that can locate the fire incident location using different search types, identify the least cost path from fire station to the incident, and identify the best places for firebreaks.

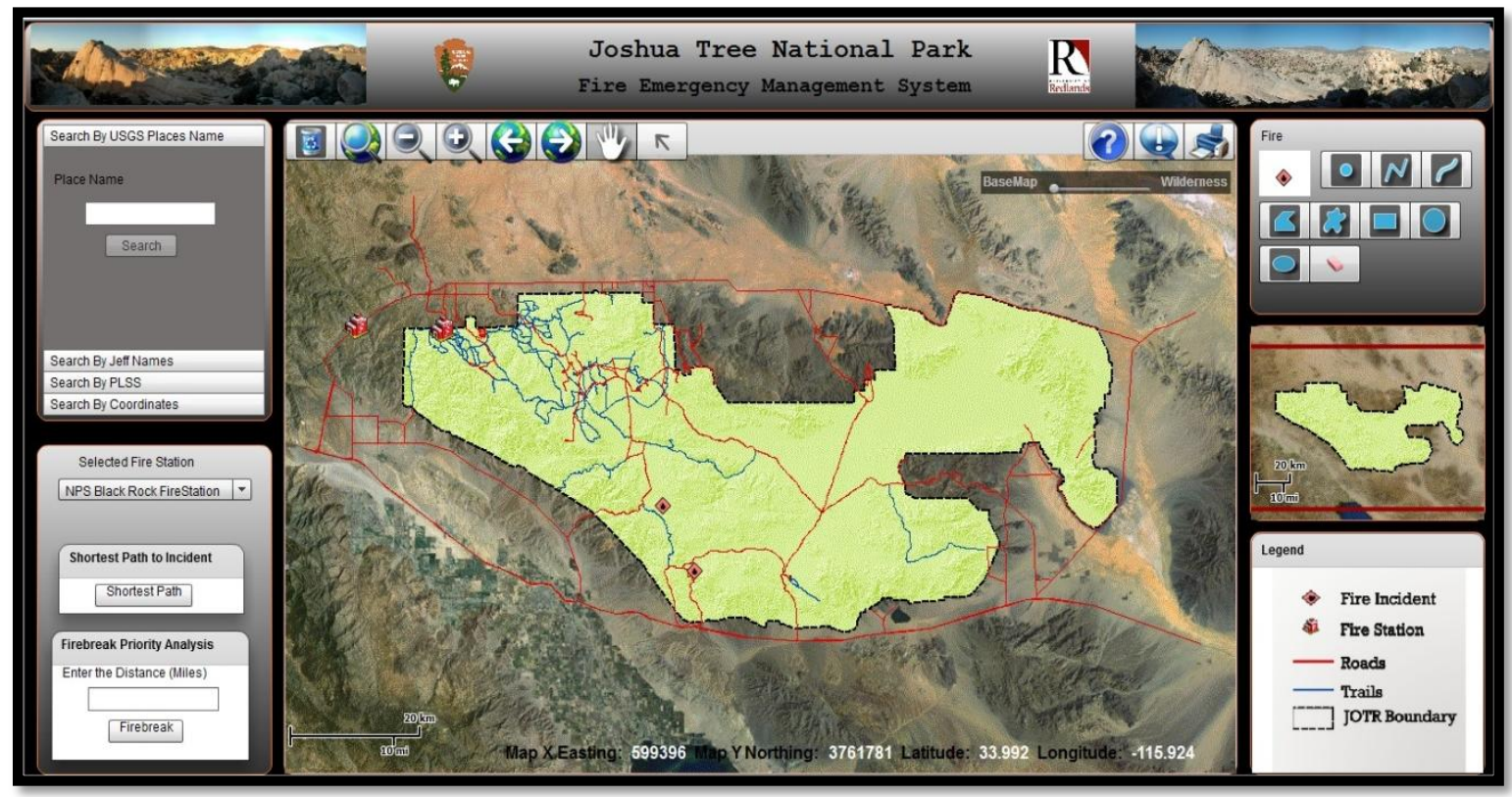

Figure 6-8 FEMS Web Application

\subsubsection{Web Application Functions Results}

The FEMS web application enables users to locate and display the fire incident on the map, and use a set of functions to assist with fire response.

\subsubsection{Where is the Fire Incident Located?}

The user can identify the fire incident location using four types of search methods: searching by USGS places names, searching by JOTR local names (Jeff names), searching by PLSS, and searching by coordinates system (latitude, longitude). Figure 69 shows two different types of search methods results to locate the fire incident location. 

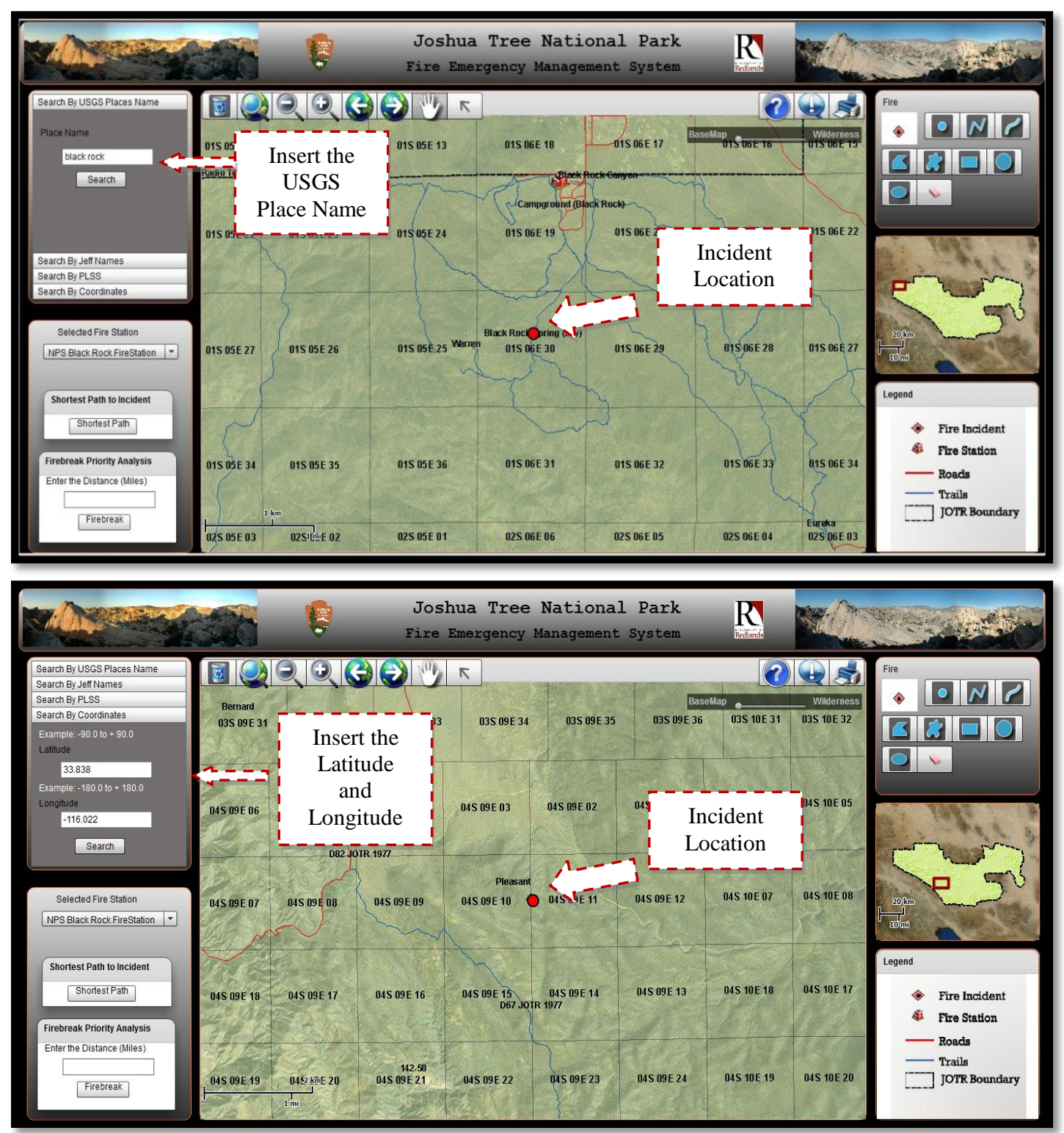

\section{Figure 6-9 Search Results}

\subsubsection{Where is the incident location stored?}

After identifing the fire incident location, the user will click the Fire Symbol to insert the incident location in the personal ArcSDE geodatabase. The user will insert the incident date, the description of the fire incident, the fire station ID, and the status of the fire incident. The fire station ID will be 1 if the NPS fire station deals with that fire incident, otherwise the user will insert 2 for BLM fire station. Figure 6-10 shows the results of this editing method. 


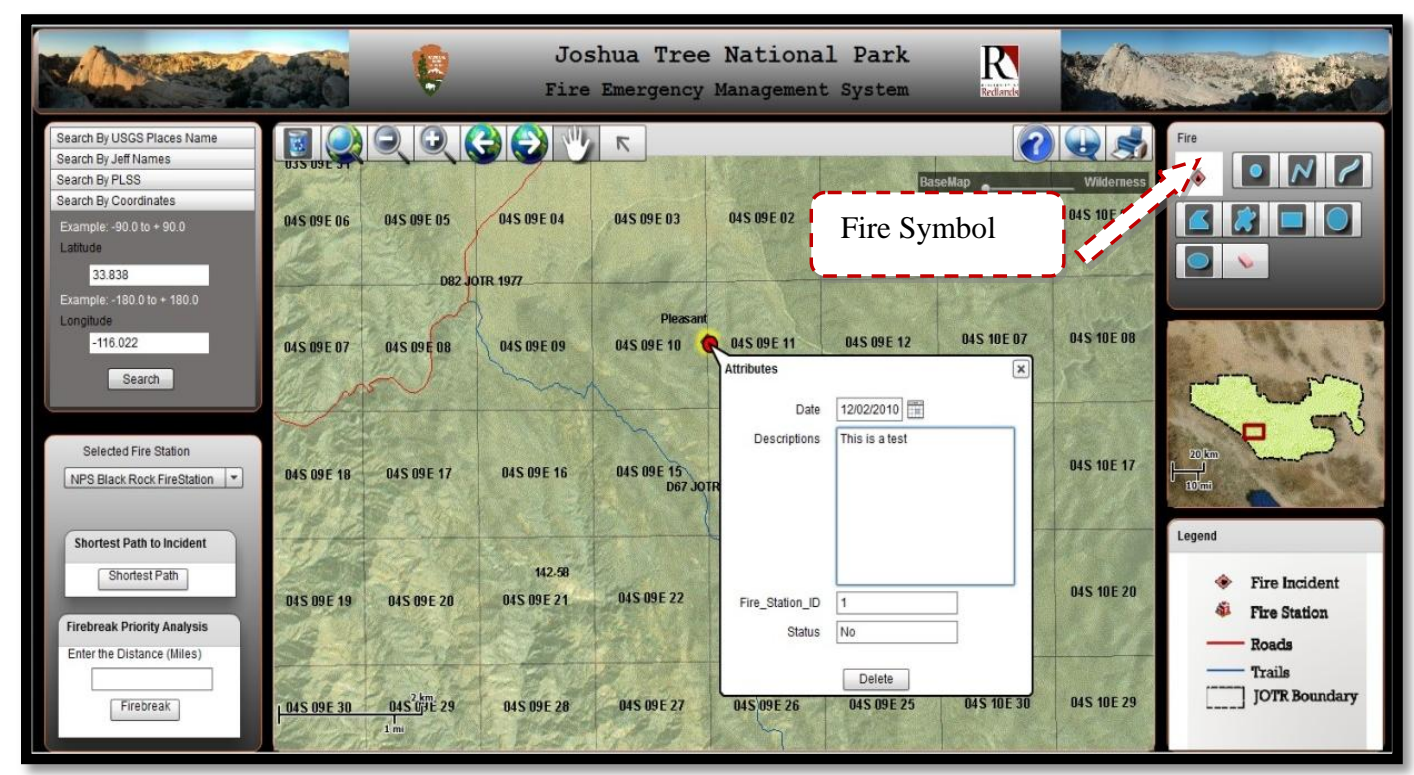

\begin{tabular}{|c|c|c|c|c|c|}
\hline OBJECTID * & Fire_Station ID & Date & Descriptions & Status & SHAPE* \\
\hline 6 & & 2 11:4200107:00:00 AM & Test & Yes & Point \\
\hline 9 & & 1 1115000107:00:00 AM & test & No & Point \\
\hline 10 & & $21115200107: 00: 000 \mathrm{AM}$ & This is a another Test & Yes & Poirt \\
\hline 11 & & $2115500107: 00000 \mathrm{AM}$ & Fire Fire Five & Yess & Point \\
\hline 16 & & 1 1180200108:00:00 AM & Five in Jeff Nane Areas & No & Point \\
\hline 17 & & 1 1180200108:00:00 AM & Coovdingtes Systen Checking & Yes & Point \\
\hline-23 & & 1 118200108:00:00 AM & This is color test & No & Point \\
\hline Incident & & $111100200108000000 \mathrm{AM}$ & The Testing the systen functions & Yess & Point \\
\hline $1=-727$ & & $1111,02000108000000 \mathrm{AM}$ & This is atest & No & Point \\
\hline
\end{tabular}

\section{Figure 6-9 Editing Results}

When the user changes the status of the fire incident to "Yes," it will be updated in the geodatabase. After changing the status value to "Yes" the next time the user accesses the web application, that incident will not be displayed. In addition, the user can select the fire incident and click the "Delete" button to delete the incident from the geodatabase.

\subsubsection{What is the shortest path to the fire from a fire station?}

The benefit of the Cost Surface Analysis tool is that it prepares the Cost Distance and Backlink raster datasets, which will be used as an input in the Least Cost Path geoprocessing tool. The user will click on the incident location, select the fire station, and click the "Shortest Path" button to run the Least Cost Path Tool. This tool will return the shortest path to the fire from the fire station, as shown in Figure 6-11. 


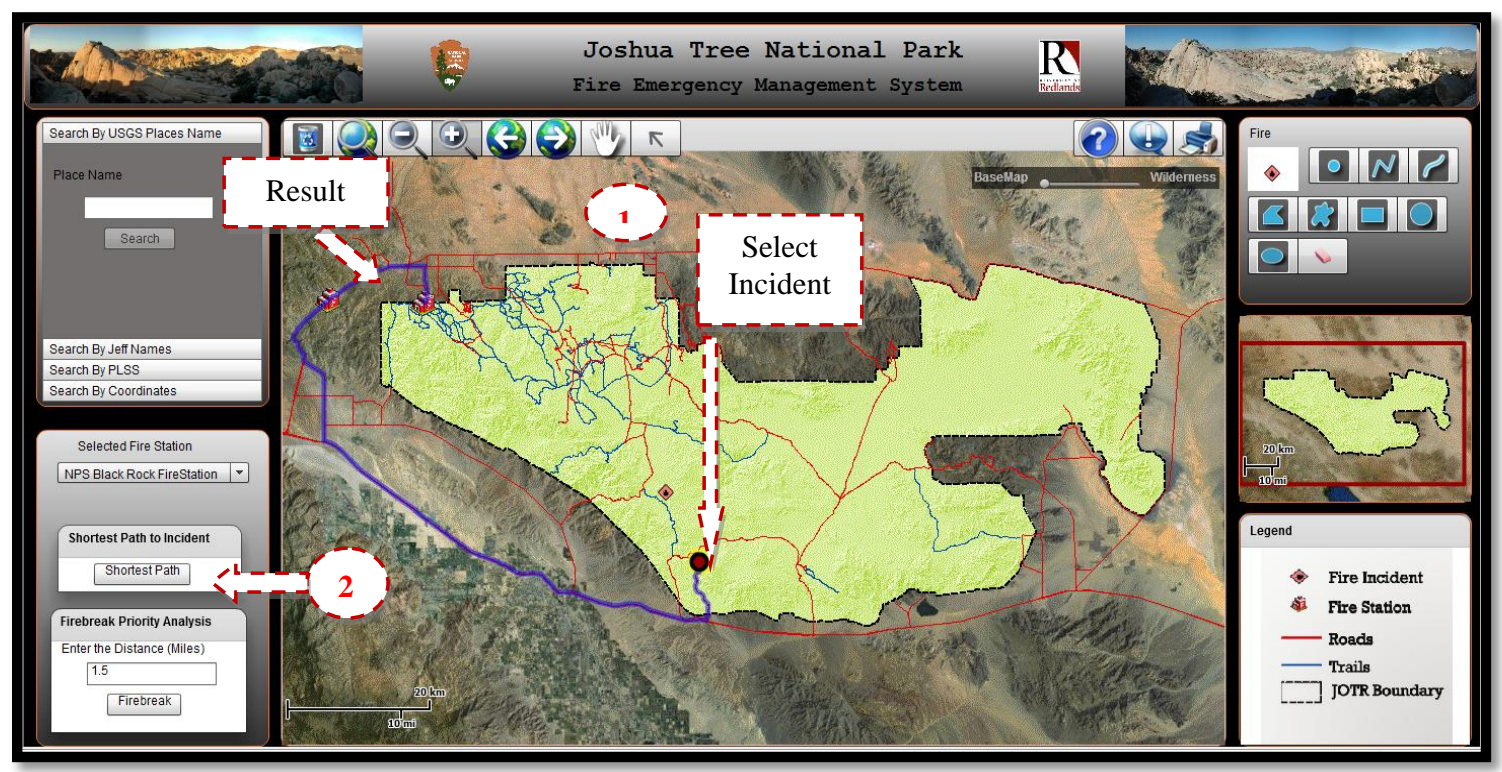

Figure 6-10 Shortest Path

\subsubsection{What are the best places for firebreak?}

The benefit of the Firebreak Preparing Data tool shown here is to use the output of this tool, which is stored in the geodatabase, as an input to the Firebreak Priority Analysis geoprocessing tool. The user will click on the incident location, insert the distance in miles, and click the "Firebreak" button to run the Firebreak Priority Analysis tool. This tool will return the analysis results, as shown in Figure 6-12.

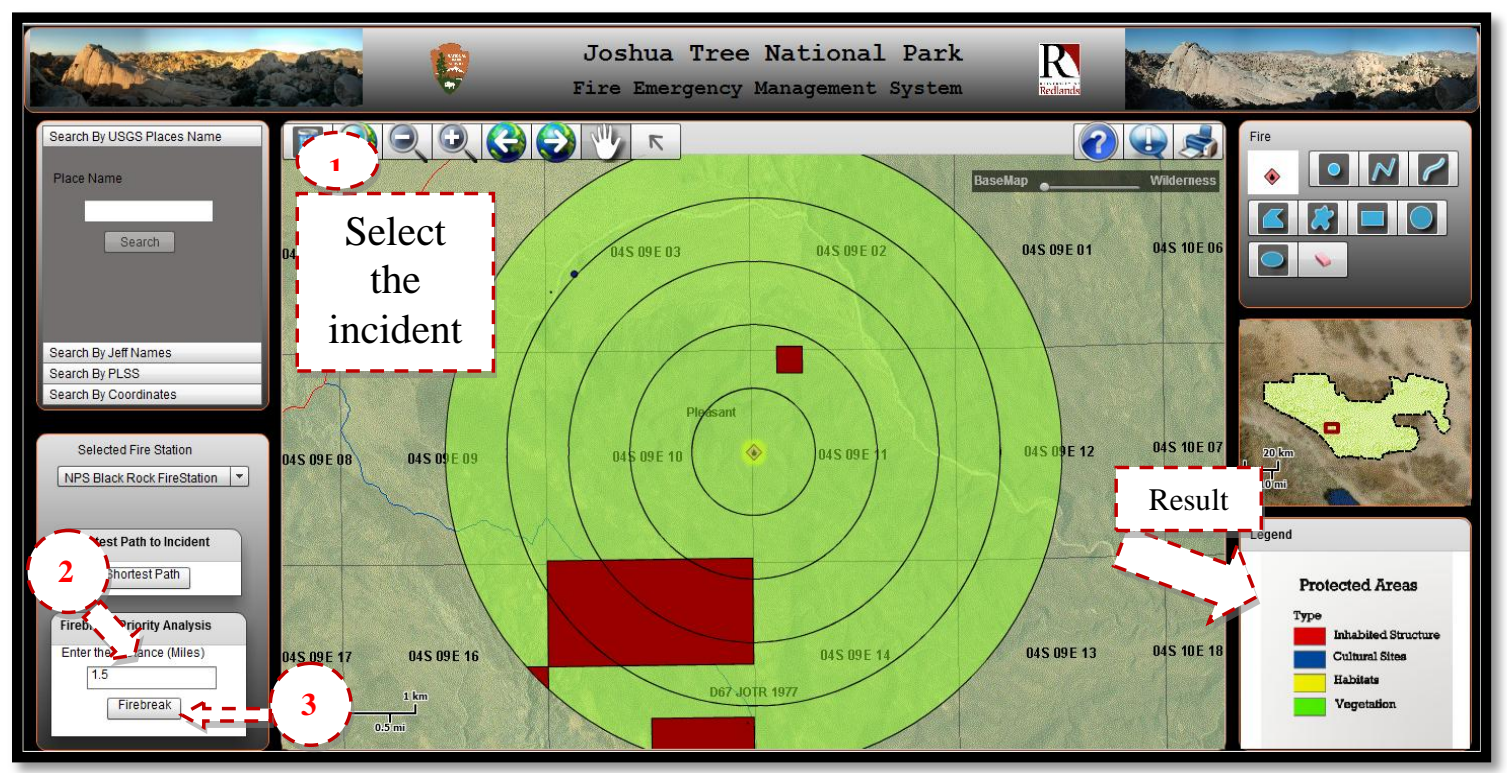

Figure 6-11 Firebreak Priority Analysis Tool Output 
When the result is displayed on the map, the legend will change, as shown in Figure 6-12. Figure 6-13 shows the benefit of using the drawing tool to help the user to decide where the firebreak should be located.

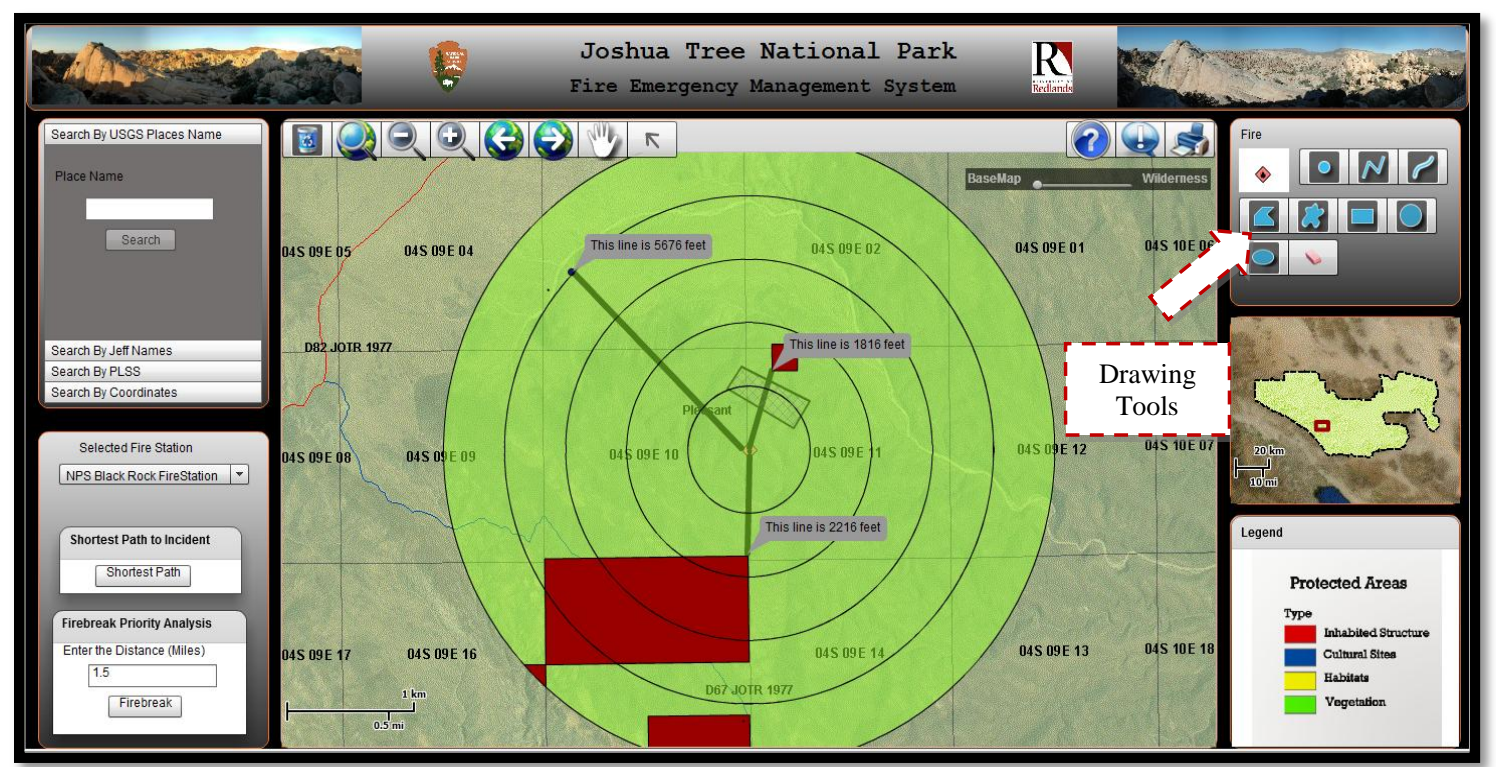

Figure 6-12 Using Drawing Tools

As the client requested, the user can know whether the fire incident is located in the wilderness area or not. Figure 6-14 shows the result of using the Slider Window to show the wilderness areas.

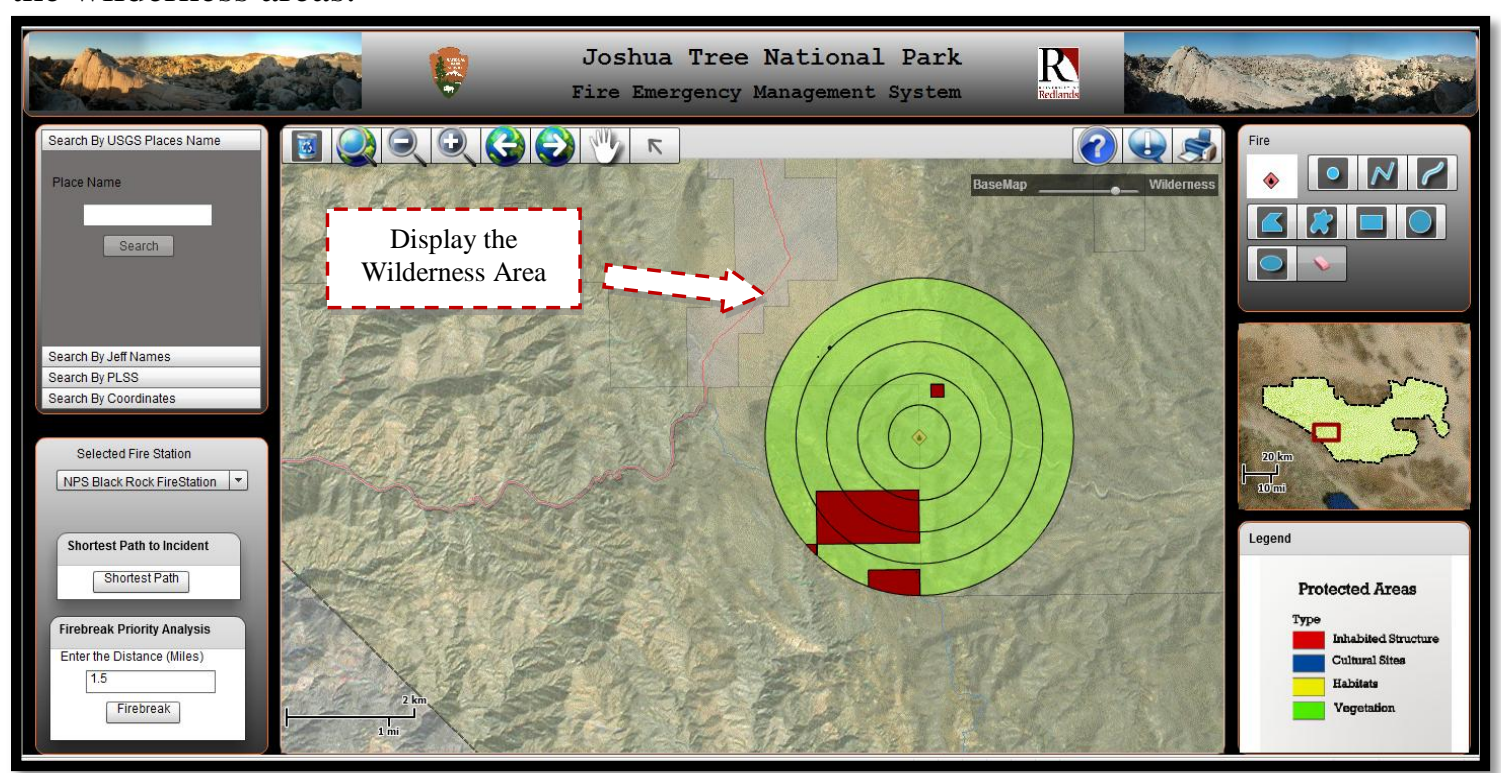

Figure 6-13 Using Slider Window To Show Wilderness Areas

\subsubsection{Other tools in the FEMS Web Application}

There are a set of tools that enable the user to interact with the application map, such as Zoom In, Zoom Out, and Map Full Extent. The user can print the displayed map by 
clicking the print button. An overview map orients the user by showing the location of the current view in the context of a larger map area.

\subsection{Summary}

This chapter discusses the results of the GIS tools developed to prepare the data for the geprocessing tools inputs. It also discusses the FEMS web application key functions that were implemented using ArcGIS Flex API. This web application enables the user to identify the fire incident location using different types of searches, identify the shortest path to the fire from the fire station, and identify the best places for the firebreak. The next chapter will outline the conclusions and future work. 


\section{Chapter 7 - Conclusions and Future Work}

The previous chapter looks at what the project carried out and displays the analysis that was performed in the web application. This chapter concludes the FEMS report, summarizes the project, and presents possible ideas for product spin-offs. The project satisfied the client's functional and non-functional requirements, and the tools and the web application are ready for operational deployment.

\subsection{Conclusion}

The overall goal of this project was to assist JOTR with more rapid response to wildfire incidents and to prioritize creation of firebreaks. To meet this goal, two Python scripting tools were developed to prepare data to be used as an input in other two-geoprocessing tools, which would run through the web application. In addition, a web-based application was developed using ArcGIS Flex API to assist with fire response.

The scripting tools were built using a Python scripting language, which can work more quickly and integrate with different systems effectively. These tools are simple to use, but the user must be familiar with the data used for each tool. All scripting tools were tested and fully functional. In addition, the web application was built using ArcGIS Flex API, which enables the user to build dynamic rich Internet applications on top of the ArcGIS Server. The web application enables the user to identify the fire incident location using different types of searches, identify the shortest path to the fire from the fire station, and identify the best places for the firebreak. It also provides the user with a set of tools that enable the user to deal with the application map, such navigation and printing tools. All web application functions were tested and found fully functional. A systematic handbook was provided to help guide the users through the functionality of the scripting tools and the FEMS web application. The client's functional and non-functional requirements were met.

\subsection{Future Work}

Because of the extensive amount of work involved in creating the tools and the web application for this project, the lack of certain collected data types, as well as ideas generated by completing this project, there are several areas for future work to expand on this project. The following sections discuss three potential tasks: enhancing the Firebreak Priority Analysis tool, evaluating fire risk, and analyzing fire spread using Python scripting or ModelBuilder.

\subsubsection{Enhancing the Firebreak Priority Analysis tool}

Since most of the data collected from the client is in point form, the results will not be accurate, as much as if the available data type is polygon. If the polygon data type is available, the weighted overlay analysis can apply in this case. In addition, the wind direction can be included as a factor to identify the best place for firebreak. 


\subsubsection{Evaluating Fire Risk}

Since fire, depends on different factors, such as temperature, humidity, sun, and vegetation type, a new scripting tool or a new function on the FEMS web application can be built to evaluate where the fire incident can be predicted depending on these factors.

\subsubsection{Analyzing Fire Spread}

A Python scripting tool or a model can be built to simulate the fire spread based on wind direction, and whether or not the cell in a raster can burn. Figure 7-1 shows the predicted spread rate of fire within five minutes, and the type of data that can be used to analyze fire spread.

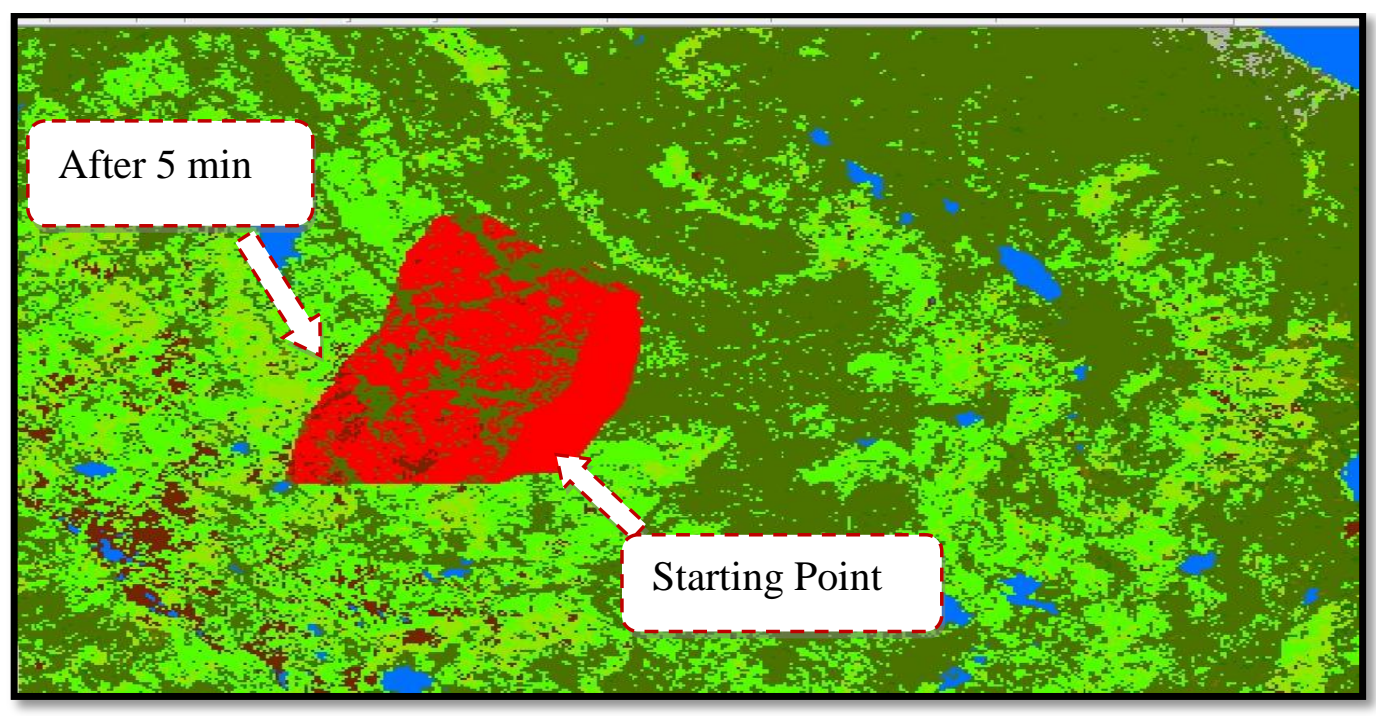

\begin{tabular}{|c|c|c|c|c|c|c|c|c|}
\hline & OBJECTID* & VALUE & COUNT & \multicolumn{3}{|c|}{ CODE_DESC } & BurnDuration & Flammability \\
\hline 1 & 1 & 11 & 245897 & \multicolumn{3}{|l|}{ Water: Open Water } & 0 & 0 \\
\hline & 2 & 12 & 1484 & \multicolumn{3}{|c|}{ Water: Perennial loe/Snow } & 0 & 0 \\
\hline & 3 & 21 & 7401 & \multicolumn{3}{|c|}{ Developed: Low Intensity Residential } & 70 & 30 \\
\hline & 4 & 23 & 486 & \multicolumn{3}{|c|}{ Developed: Commercial/ndustria//Transportation } & 20 & 20 \\
\hline & 5 & 31 & 9077 & \multicolumn{3}{|l|}{ Barren: Roo/Sand/Clay } & 0 & 0 \\
\hline & 6 & 41 & 2504 & \multicolumn{3}{|c|}{ Forested Upland: Deciduous Forest } & 80 & 50 \\
\hline & 7 & 42 & 285805 & \multicolumn{3}{|c|}{ Forested Upland: Evergreen forest } & 90 & 40 \\
\hline & 8 & 43 & 6391 & \multicolumn{3}{|c|}{ Forested Upland: Mixed Forest } & 85 & 45 \\
\hline & 9 & 51 & 99179 & \multicolumn{3}{|l|}{ Shrubland: Shrubland } & 40 & 80 \\
\hline & 10 & 71 & 29082 & \multicolumn{3}{|c|}{ Herbsceous Upland: Grassland/Herbaceous } & 10 & 90 \\
\hline & 11 & 85 & 213 & \multicolumn{3}{|c|}{ Cultivated: Urban Grasses } & 10 & 90 \\
\hline & 12 & 91 & 39 & \multicolumn{3}{|l|}{ Wetland: Wooded } & 80 & 20 \\
\hline & 13 & 92 & 778 & \multicolumn{3}{|l|}{ Wetland: Herbaceous } & 20 & 60 \\
\hline & Record: is 1 & 1 & ओ & how: All Selected & Records (0 out of 13 Selected) & Options . & & \\
\hline
\end{tabular}

Figure 7-1 Analyze Fire Spread (ESRI, Performing Analysis with ArcGIS Desktop, 2009) 
Figure 7-1 also shows the land cover data type and the flammability value for each type. For example, grasses are more flammable than deciduous forests and water is not flammable at all (ESRI, Performing Analysis with ArcGIS Desktop, 2009).

\subsection{Summary}

Using GIS and Flex API, a set of tools and web application were developed to aid the JOTR fire station to conduct accurate analysis, help the fire dispatch center reduce the response time to fires, and to preserve the park from the fire threat. Future work could add more variables to the framework of this project to improve its performance and analytical capabilities. This project successfully fulfilled the client's functional and nonfunctional requirements, with potential improvements identified for future work. 



\section{Works Cited}

Amdahl, G. (2001). Disaster Response: GIS for Public Safety. Redlands: ESRI.

Arctur, D., \& Zeiler, M. (2004). Design Geodatabases. Redlands: ESRI Press.

Balstrom, T. (2002). On Identifying the Most Time-saving Walking Route in a Trackless Mountainous Terrain. Danish Journal of Geography 102: 51-58.

Boehm, B. (1988). Spiral Model. Retrieved September 14, 2010, from Wikipedia: http://en.wikipedia.org/wiki/Spiral_model

Chatham, B., \& Britton, R. (2002). Emergency Management \& GIS Integration at the City of Fort Worth. City of Fort Worth, Texas.

Cova, T. (1999). GIS in Emergency Management. New York.

Dawson, M. (2010). Python Programming. Course Tecnology Center Learning.

Drabek, T. E., \& Hoetmer, G. J. (1991). Emergency Management: Principles and Practice for Local Government. Washington DC: International City Managers Association.

Drake-Hillyard, P. (n.d.). Lost Horse Fire at Joshua Tree Consumes 450 Acres. Retrieved October 4, 2010, from http://laist.com/2009/05/26/lost_horse_fire_at_joshua_tree.php

ESRI. (2009). ArcGIS 9.3.1 Help. Redlands.

ESRI. (n.d.). Creating and Analyzing Surface Using ArcGIS Spatial Analyst. Redlands, CA, USA.

ESRI. (2008). Geographic Information systems Providing the Platform for Comprehensive Emergency Management. Redlands: ESRI.

ESRI. (n.d.). Geoprocessing Raster Data Using ArcGIS Spatial Analyst. Redlands, CA, USA.

ESRI. Mapping the Future of Public Safety. Redlands: ESRI.

ESRI. (2009). Performing Analysis with ArcGIS Desktop. Redlands: ESRI Press.

ESRI. (2009). Performing Analysis with ArcGIS Desktop. Redlands: ESRI Press.

ESRINEA. (2009). Geographic Security System Suites. Retrieved October 2, 2010, from ESRI Northeast Africa: http://www.esrinea.com/index.aspx?id=120

Johnson, R. (2000). GIS Technology for Disasters and Emergency Management. Redlands: ESRI.

Kwan, M.-P., \& Lee, J. (2003). Emergency response after 9/11:The Potential Of RealTime 3D GIS For Quick Emergency Response In Micro-Spatial Envirmonment.

Longley, P., \& Batty, M. (2003). Advanced Spatial Analysis. Redlands: ESRI Press.

Michael, G. (2000). GIS and Transportation: Status and Challenges.

Peng, Z.-R., \& Tsou, M.-H. (2003). Internet GIS Distributed Geographic Information Services for the Internet and Wireless Networks. (pp. 1-35). Hoboken, NJ: John Wiley \& Sons, Inc.

Peters, D. (2008). Building a GIS - System Architecture Design Strategies for Managers . Redlands: ESRI Press.

Steiner, F., McSherry, L., \& Cohen, J. (2000). Land Suitability Analysis for the Upper Gila River watershed. Arizona.

Taverna, K., Halbert, J. E., \& Hines, D. M. (2000). Habitat Suitability Analysis for the Central Appalachians. Charlottesville. 
Tobler, W. (1993). Three Presentations on Geographical Analysis and Modeling . Santa Barbara: University of California, National Center for Geographic Information and Analysis.

Tomlinson, R. (2007). Thinking About GIS for Managers. Redlands, CA: ESRI. 


\section{Appendix A. Python Scripting Tools}

\section{Python Script Code 1: Cost Surface Analysis Tool}

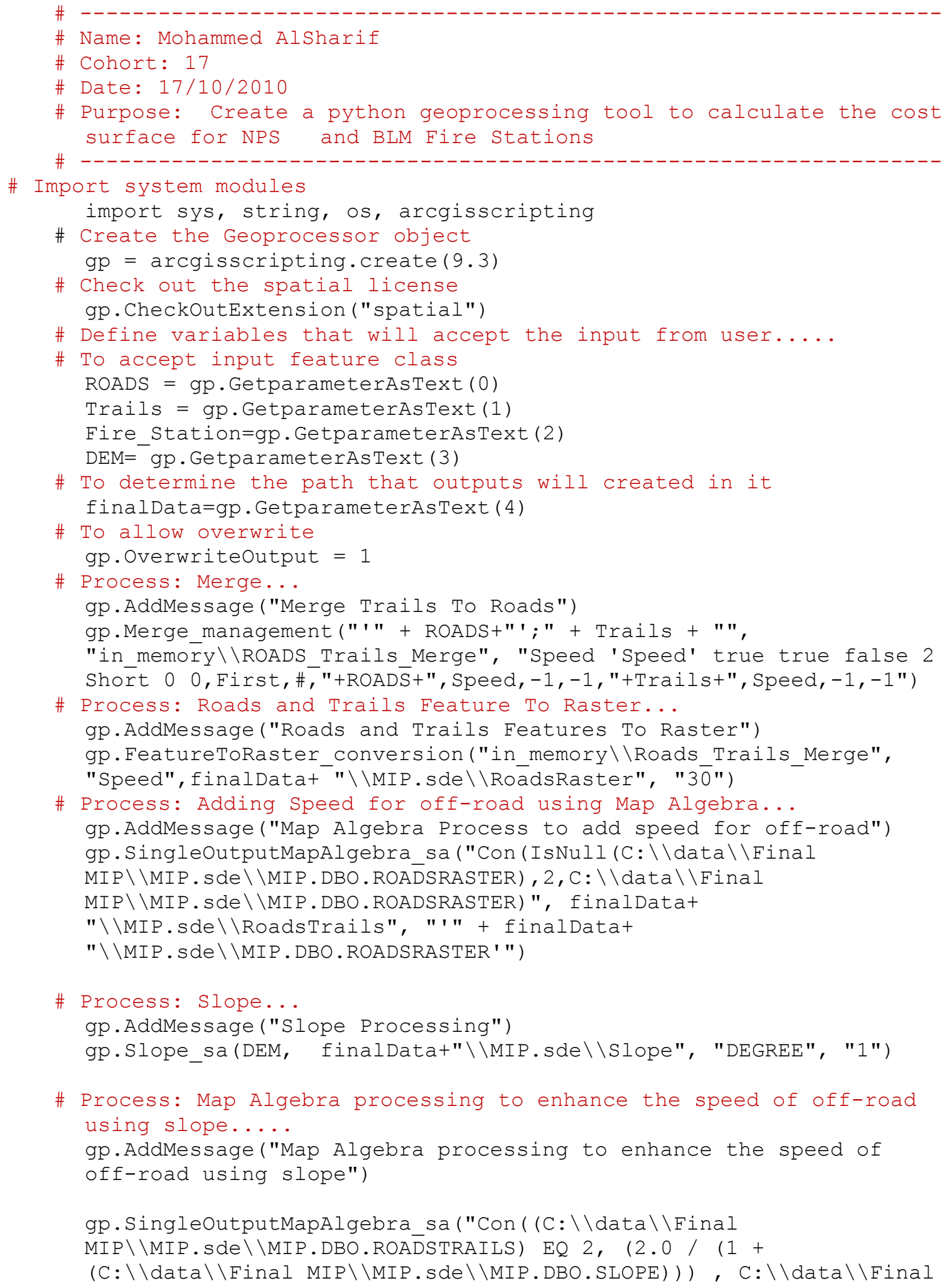


MIP \\MIP.sde \\MIP.DBO.ROADSTRAILS)", finalData+

" \\MIP.sde $\backslash$ Travelspeed ", "'" + finalData+

" $\backslash$ MIP.sde $\backslash$ MIP.DBO.RoadsTrails';'" + finalData+

" \\MIP.sde \\MIP.DBO.Slope'")

\# Process: Map Algebra to convert the speed into time...

gp. AddMessage ("Map Algebra to convert the speed into time")

gp.SingleoutputMapAlgebra_sa(" (1.0 / ( (C: \\data \\Final

MIP \MIP.sde $\backslash$ Travelspeed ) * 26.82))", finalData+

"\\MIP.sde \\ravelCost", "'" + finalData+

"\\MIP.sde \\TravelSpeed '")

\# Process: SelectnPS...

gp.AddMessage("Selecting the NPS fire station")

gp.Select_analysis(Fire_Station, "in_memory \\NPS_FS", "\"Agency "

$=$ 'NPS'")

\# Process: Cost Distance for NPS...

gp.AddMessage("Calculating the cost Distance")

gp. CostDistance_sa("in_memory \\NPS_FS", finalData+

" $\backslash \backslash$ MIP.sde $\backslash \backslash$ MIP.DBO. Trávelcost",

finalData+" \\MIP.sde $\backslash$ NPS_TravelTime", "",

finalData+" \\MIP.sde \\NPS_BackLink")

\# Process: Select BLM...

gp.AddMessage("Selecting BLM fire station")

gp.Select_analysis(Fire_Station, "in_memory \\BLM_FS", "\"Agency "

$=$ 'BLM'")

\# Process: Cost Distance for BLM...

gp.AddMessage ("Cost Distance processing for BLM")

gp. CostDistance sa("in memory \\BLM FS", finalData+

" $\backslash$ MIP.sde $\backslash$ MIP.DBO.TrāvelCost", fínalData+

"\\MIP.sde $\backslash$ BLM TravelTime"," ", finalData+

"\\MIP.sde \\BLM_BackLink")

\section{Python Script Code 2: Firebreak Preparing Data Tool}

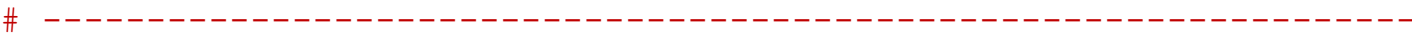

\# Name: Mohammed AlSharif

\# Cohort: 17

\# Date: 28/9/2010

\# Purpose: Create a python geoprocessing tool to find the final output of the Priority analysis (firebreak)

\#------------------------------------------------------------------

\# Import system modules

import sys, string, os, arcgisscripting

\# Create the Geoprocessor object gp = arcgisscripting. create (9.3)

\# Define variables that will accept the input from the user...

\# To accept multi-input features classess Inhabited_Structure_inputs=gp.GetparameterAsText (0)

Cultural_sites_inputs= gp.GetparameterAsText (1)

Habitats_inputs $\bar{s}=$ gp.GetparameterAsText (2)

\# To accept input feature class vegetation_input $=$ gp. GetparameterAsText (3)

\# To determine the path that outputs will created in it finalData $=$ gp. GetparameterAsText (4) 
\# To allow overwrite

gp. Overwriteoutput $=1$

\# Inhabited Structure Process variables

Inhabited_Structure =finalData+

" \\MIP.sde $\backslash \backslash$ MIP.DBO.FireBreak \\Inhabited_Structure"

Inhabited_Dissolve =finalData+

" \\MIP.sde $\backslash \backslash M I P . D B O . F i r e B r e a k \backslash \backslash$ Inhabited_Dissolve"

\# Cultural Sites Process variables

Cultural_Sites = finalData+

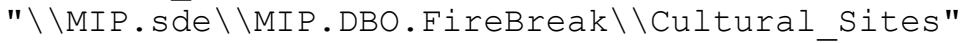

Cultural Dissolve = finalData+

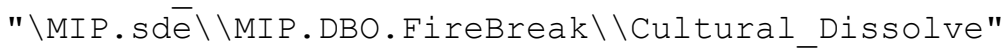

\# Habitats Process variables

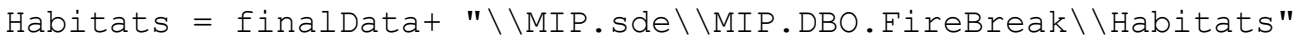

Habitats Dissolve = finalData+

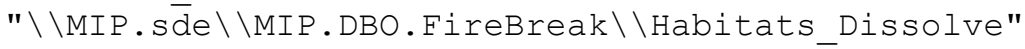

\# Union All Process Variables

protected Areas =finalData+

" \\MIP.sde $\backslash \backslash M I P . D B O . F i r e B r e a k \backslash \backslash$ protected_Areas"

\# Vegetation Process Variables

Vegetation Dissolve = finalData+

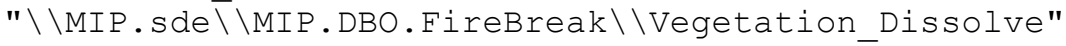

\# Habitats Processing

gp.AddMessage ("Habitats Processing ....." )

\# Split the input list

habitats inputs list=Habitats inputs.split(";")

for habitatsFC in habitats_inputs_list:

\# Gets class name from full path

\# lstrip to get the name after "." and it was used here because the feature class name include the MIP.DBO.FeatureName so the function will return the FeatureName

\# the splitext to split the name in to a pair root and ext for example to remove. shp from shapefile name

habitatsFC=habitatsFC.strip (" '")

fcName=os.path.splitext (habitatsFC) [1] .lstrip (".")

fcName=fcName.strip ("'")

\# Habitats Buffer processing .....

\# To add a message when the tool is executing

gp.AddMessage (fcName + " Habitats Buffer Processing ..." )

\# To store the fcNameBuffer in the PC memory fcNameBuffer="in_memory \\" + fcName + "_Buffer"

\# Using try and except to complete the program working if there is any error occur

try:

gp.Buffer_analysis(""+ habitatsFC +"", fCNameBuffer, "5 except: Meters", "FULL", "ROUND", "NONE", " )

gp.AddMessage ("Error during the buffer processing...")

\# Process: Habitats Union...

gp.AddMessage ("Habitats Union Processing ....." )

gp.Union_analysis("'in_memory \\mammal_Buffer'

\#; 'in_memory \tort_sight__Buffer' \#;'iñ_memory \\snakes_Buffer' 
\#;'in memory \guzzler Buffer'

\#; 'in memory $\backslash \backslash$ Amphibiān Reptile Buffer'

\#;'in memory \\Pool_locations_buffer' \#", Habitats, "ALL", "", "GAPS")

\# Process: Habitat Dissolve...

gp.AddMessage("Habitats Dissolve Processing ....")

gp.Dissolve management(Habitats, Habitats_Dissolve, " , " ", "MULTI PART", "DISSOLVE LINES")

\# Process: Habitats Add Field...

gp.AddMessage("Habitats Add Field Processing ... ")

gp.AddField_management (Habitats_Dissolve, "Habitats_Types",

"TEXT", " ", " " ", " ", "NULLABLEE", "NON_REQUIRED", " ")

\# Process: Habitat Calculate Field...

gp.AddMessage("Habitats Calculate Field Processing....")

gp.CalculateField_management(Habitats_Dissolve, "Habitats_Types",

"\"Habitats\", "PYTHON_9.3", " ")

\# Inhabited Structure Processing

gp.AddMessage ("Inhabited Structure Processing .....")

\# Split the input list

Inhabited_Structure_inputs_list=Inhabited_Structure_inputs.split ( ";")

for inhabitedFC in Inhabited Structure inputs list:

\# Gets Class name from full path

\# lstrip to get the name after "." and it was used here because the feature class name include the MIP.DBO.FeatureName so the function will return the FeatureName

\# the splitext to split the name in to a pair root and ext for example to remove. shp from shapefile name

inhabitedFC=inhabitedFC.strip ("' ")

fcName=os.path.splitext (inhabitedFC) [1].lstrip (".")

fcName=fcName.strip ("' ")

\# Inhabited Structure Buffer processing ....

\# To add a message when the tool is executing

if fcName != "inholding":

gp.AddMessage (fcName + " Inhabited Structure Buffer Processing")

\# To store the fcNameBuffer in the PC memory fcNameBuffer="in_memory \\" + fcName + "Buffer"

\# Using try and except to complete the program working if there is any error occur

try:

gp.Buffer_analysis(""+ inhabitedFC +"", fcNameBuffer, "25

Meters","FULL", "ROUND", "NONE", " ")

except:

else:

gp.AddMessage("Error during the buffer processing...")

inholding= inhabitedFC

gp.AddMessage ("Creater Inholding layer")

gp.MakeFeatureLayer_management (inholding, "inholding")

\# Process: Inhabited Structure Union...

gp.AddMessage ("Inhabited Structure Union Processing ....")

gp.Union_analysis ("inholding ; 'in_memory \\campground_Buffer' \# ;

'in_memory \\Structure_Building_Buffer' \#", Inhabited_structure,

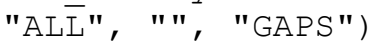

\# Process: Dissolve...

gp.AddMessage("Inhabited Structure Dissolve Processing ...") 
gp.Dissolve_management(Inhabited_Structure, Inhabited_Dissolve, " , " ", "MULTTI_PART", "DISSOLVE_L_LNES")

\# Process: Add Field...

gp.AddMessage("Inhabited Structure Add Field Processing ....")

gp.AddField_management (Inhabited_Dissolve,

"Inhabited_Structure_Types", "TEX̄T", " , " , " , " , "NuLLABLE", "NON_REQUIRED", " ")

\# Process: Calculate Field...

gp.AddMessage("Inhabited Structure Calculate Field Processing")

gp.CalculateField_management(Inhabited_Dissolve,

"Inhabited_Structüre_Types", "\"Inhabited Structure\"",

"PYTHON 9. $3 ", "$ ")

\# Cultural Sites Processing

gp.AddMessage ("Cultural Sites Processing ....." )

\# Split the input list

Cultural_Sites_inputs_list=Cultural_Sites_inputs.split(";")

for culturalFC in cultural_sites_inputs_list:

\# Gets Class name from full path

\# lstrip to get the name after "." and it was used here because the feature class name include the MIP.DBO.FeatureName so the

function will return the FeatureName

\# the splitext to split the name in to a pair root and ext for

example to remove. shp from shapefile name

culturalFC=culturalFC.strip ("'")

fcName=os.path.splitext (culturalFC) [1].lstrip (".")

fcName=fcName.strip ("' ")

\# Cultural Site Buffer processing ....

\# To add a message when the tool is executing gp.AddMessage (fcName + " Cultural sites Buffer Processing")

\# To store the fcNameBuffer in the PC memory fcNameBuffer="in memory \\" + fcName + " Buffer"

\# Using try and except to complete the program working if there is any error occur

try:

gp.Buffer analysis(""+ culturalFC +"", fcNameBuffer, "25

Meters", "FULL", "ROUND", "NONE", " ")

except :

gp.AddMessage("Error during the buffer processing...")

\# Process: Cultural Sites Union...

gp.AddMessage ("Cultural Sites Union Processing .....")

gp.Union_analysis("'in_memory \Water_Areas_Buffer'

\#; 'in_memory \\JOTR_Minēs_Buffer' \#; 'in_memory \JJOTR_Mills_Buffer' \#; 'in_memory $\backslash \backslash$ Histōric_Pİaces_Buffer' \#", Cultural_šstes, "ALL", " , " "GAPS")

\# Process: Cultural Sites Dissolve...

gp.AddMessage("Cultural Sites Dissolve Processing ....")

gp.Dissolve_management(Cultural_Sites, Cultural_Dissolve, "", "", "MULTI_PART", "DISSOLVE_LINES")

\# Process: Cultural Sites Ädd Field...

gp.AddMessage("Cultural Sites Add Field Processing ....")

gp.AddField management (Cultural Dissolve, "Cultural_Sites_Types", "TEXT", " , " , " " ", "NULLABLE", "NON_REQUiRED", " ")

\# Process: Cultural Sites Calculate Field...

gp.AddMessage("Cultural Sites Calculate Field Processing.... ")

gp.CalculateField_management (Cultural_Dissolve,

"Cultural_Sites_Tȳpes", "\"Cultural Sītes\", "PYTHON_9.3", "") 
\# Vegetation Processing..... gp.AddMessage("Vegetation Processing .....")

\# Process: Vegetation Dissolve...

gp.AddMessage("Vegetation Dissolve Processing...." )

gp.Dissolve_management(vegetation_input, Vegetation_Dissolve, "", " ", "MULTI_PART", "DISSOLVE_LINES")

\# Process: Végetation Add Field ... gp.AddMessage("Vegetation Add Field Processing..." ) gp.AddField management (Vegetation Dissolve, "Veg_Types", "TEXT", " , " ", ", " ", "NULLABLE", "NON_E_RQUIRED", " ")

\# Process: Vegetation Calculate Fielld...

gp.AddMessage ("Vegetation Calculate Field Processing...." )

gp.CalculateField_management(Vegetation_Dissolve, "Veg_Types", "\"Vegetation\" ", "PYTHON_9.3", " ")

\# Process: ICHV Union...

gp.AddMessage("Inhabited, Cultural, Habitats and Vegetation Union Processing")

gp. Union analysis("'" + finalData +

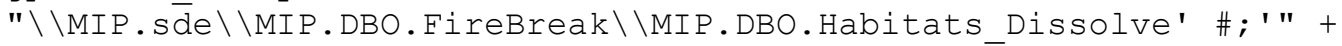
finalData +

$\backslash \backslash$ MIP.sde $\backslash$ MIP.DBO.FireBreak \\MIP.DBO.Inhabited_Dissolve' \# ; " + finalData +

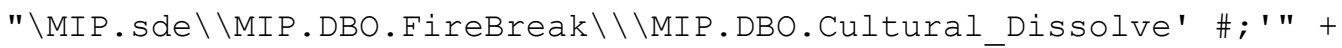
finalData +

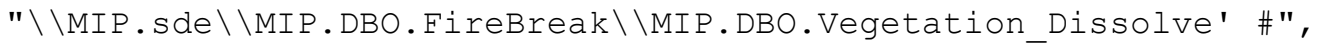
protected_Areas, "ALL", " ", "GAPS")

\# Process: Inhabited Cultural habitats Veg Add Field... gp.AddMessage("Inhabited, Cultural, Habitats and Vegetation Add Field Processing...." ) gp.AddField_management (protected_Areas, "Final_Types", "TEXT", " , " ", " "_" , "NULLABLE", "NON_REQUIRED", " ")

\# Process: Inhabited Cultural habitats Veg Calculate Field... gp.AddMessage("Inhabited, Cultural, Habitats and Vegetation Calculate Field Processing...." )

gp.CalculateField_management (protected_Areas, "Final_Types",

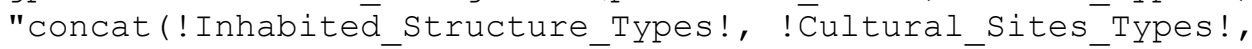
! Habitats_Types!, !Veg_Types!)", "PYTHON_9.3", "def concat (*args) : $\bar{\backslash} \backslash \mathrm{n}$ retval $=\backslash " \backslash " \backslash \backslash \mathrm{n}$ sep $=\backslash "{ }_{-} \backslash " \backslash \backslash \mathrm{n}$ for $t$ in args $: \backslash \backslash \mathrm{n} \quad \mathrm{s}=\mathrm{str}(\mathrm{t})$.strip ()$\backslash \backslash \mathrm{n} \quad$ if $\mathrm{s}<>\quad$ ' $: \backslash \backslash \mathrm{n}$ retval $+=$ sep $+s \backslash \backslash n$ return retval.lstrip (sep) \\n")

\section{Python Script Code 3: Least Cost Path Script Tool}

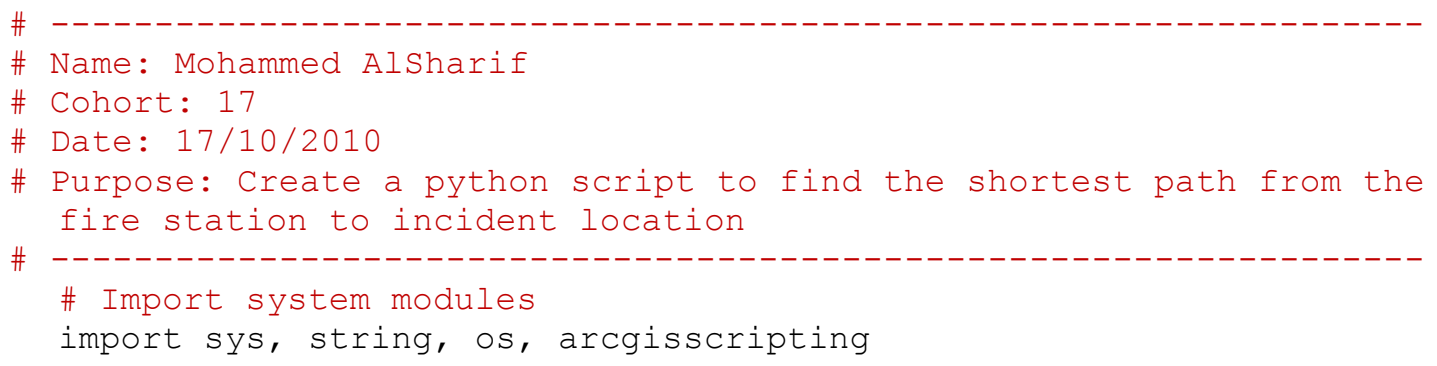


\# Create the Geoprocessor object

gp = arcgisscripting.create (9.3)

\# Check out any necessary licenses

gp. CheckOutExtension ("spatial")

\# To accept the fire incident location

Incident=gp. GetparameterAsText (0)

\# To accept the which fire station will participate in the incident FireCostSurface=gp. GetparameterAsText (1)

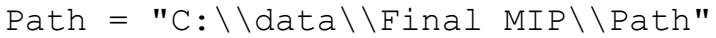

PathLine = "C: \\data $\backslash \backslash$ Final MIP \\PathLine.shp"

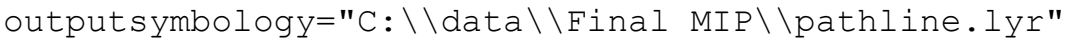

\# The cost surface for both BLM and NPS fire station

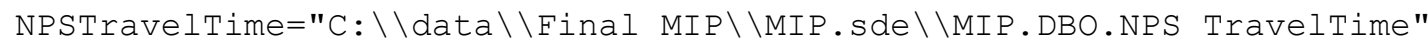

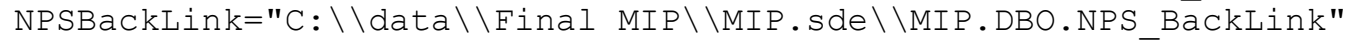

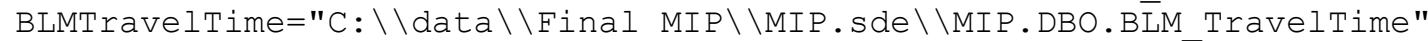

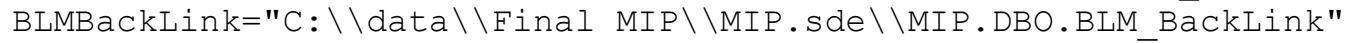

\# To check which fire station and run the cost path process to find the shortest path to fire incident

if FireCostSurface=="1":

gp.CostPath_sa(Incident, NPSTravelTime, NPSBackLink, Path, "EACH_CELL", "OBJECTID")

gp.RasterToPolyline_conversion(Path, PathLine, "ZERO", "0", "SIMPLIFY", "VALUE" )

else:

gp.CostPath_sa(Incident, BLMTravelTime, BLMBackLink, Path,

"EACH_CELL", "OBJECTID")

gp.RasterToPolyline_conversion(Path, PathLine, "ZERO", "0",

"SIMPLIFY", "VALUE")

\# to set the symbology

gp. SetParameterAs Text (1, outputsymbology)

\section{Python Script Code 4: Firebreak Priority Analysis} Tool

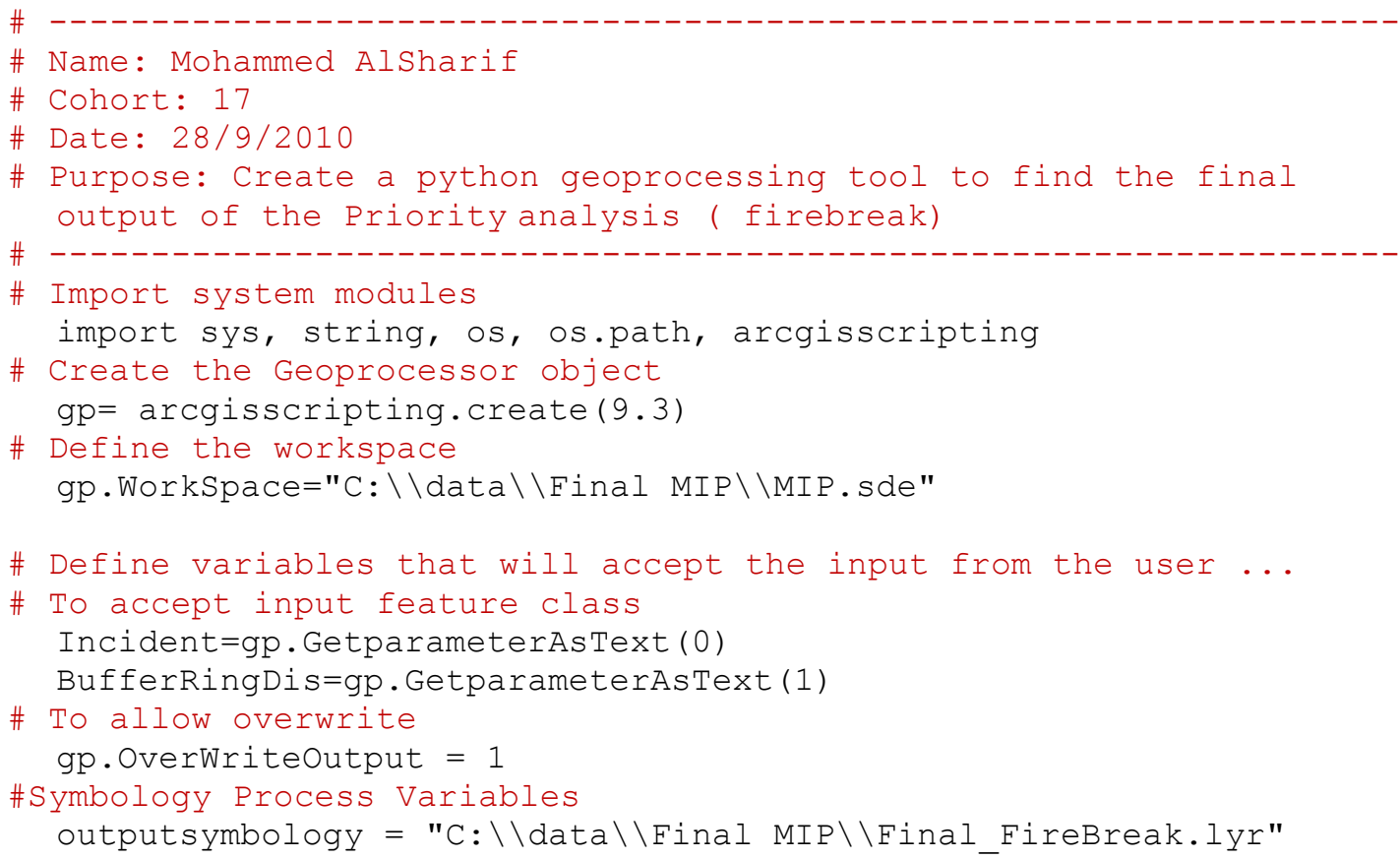


FireBreak_Symbology_Final_Layer = "C: \\ata \\Final

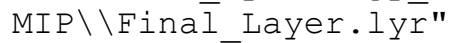

\#Define Variables

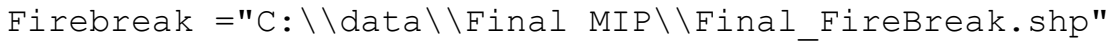

\# Process: Multiple Ring Buffer Incident...

gp.MakeFeatureLayer_management (Incident," IncidentFL")

gp. CopyFeatures manägement ("IncidentFL", "in memory \incidentFC")

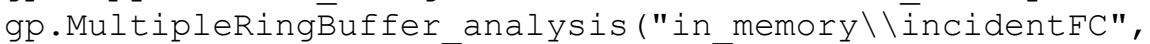

"in_memory\\Incident_Multiple_Buffēer", BufferRingDis, "Miles",

"distance", "ALL", "FULL")

\# Process: Intersect Vegetation, ICH with Incident...

gp.AddMessage("Intersect Vegetation, Inhabited, Cultural and

Habitats with Incident multiple Buffer ring Processing...")

gp.Intersect_analysis ("'in_memory \Incident_Multiple_Buffer' \#;'"

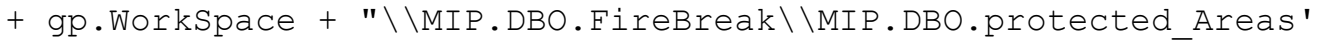

\#", Firebreak, "ALL", "", "INPUT")

\# Process: Apply Symbology From Layer...

gp. SetParameterAsText (1, outputsymbology) 


\section{Appendix B. Web GIS Application Code}

The following programming code was used to develop a FEMS web application using Flex API. All GIS functions and user interface were created by this code.

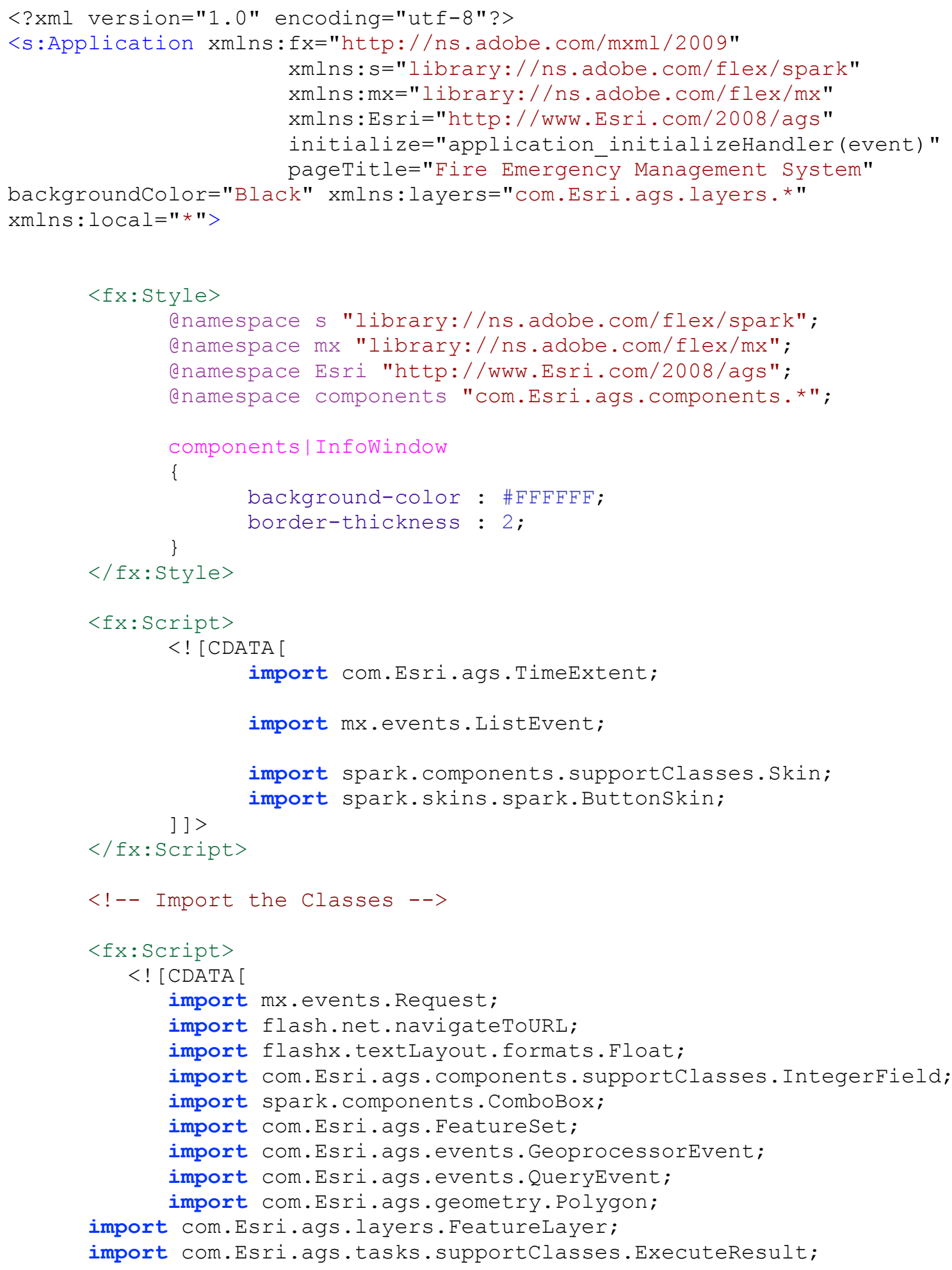




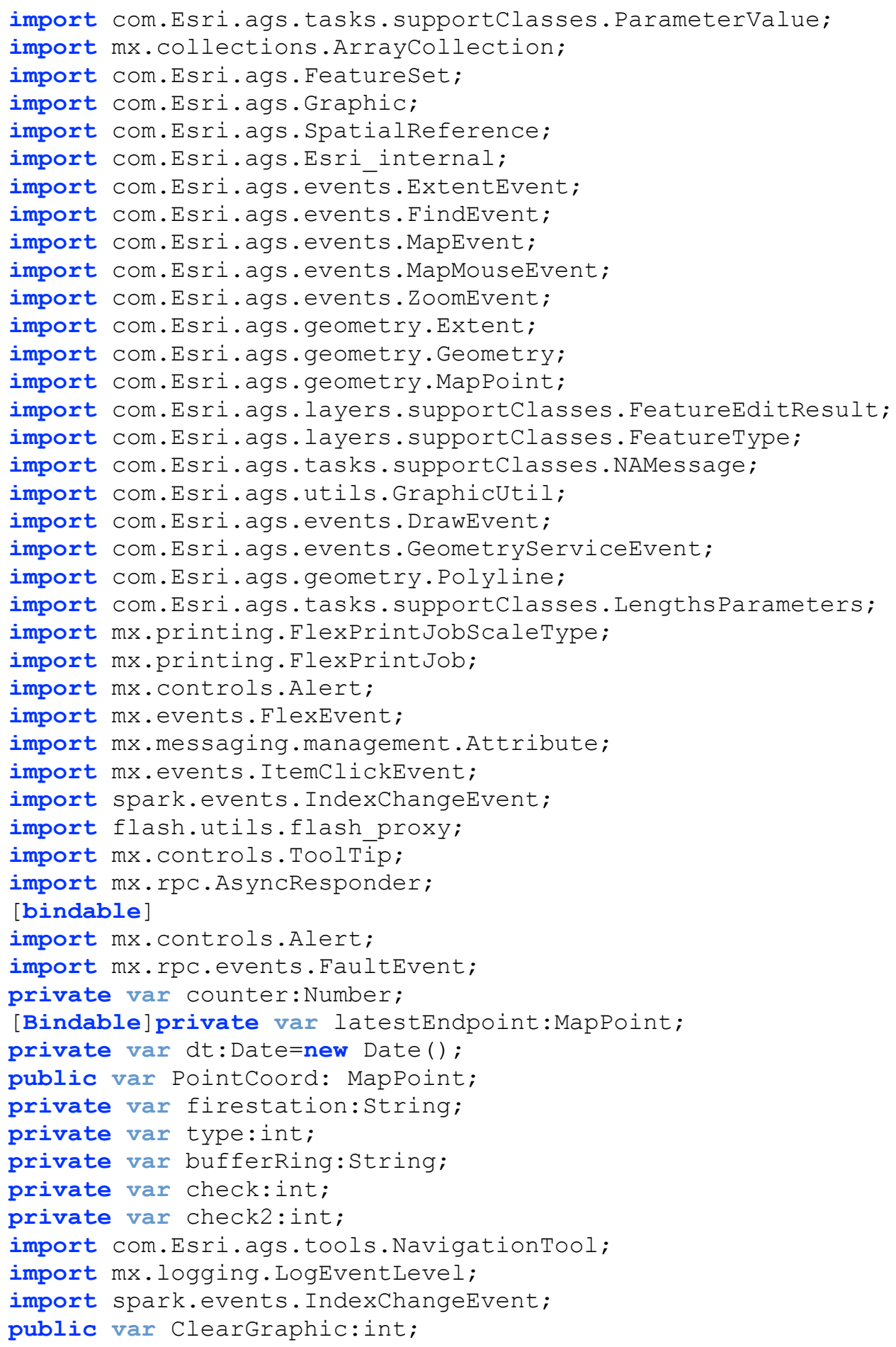




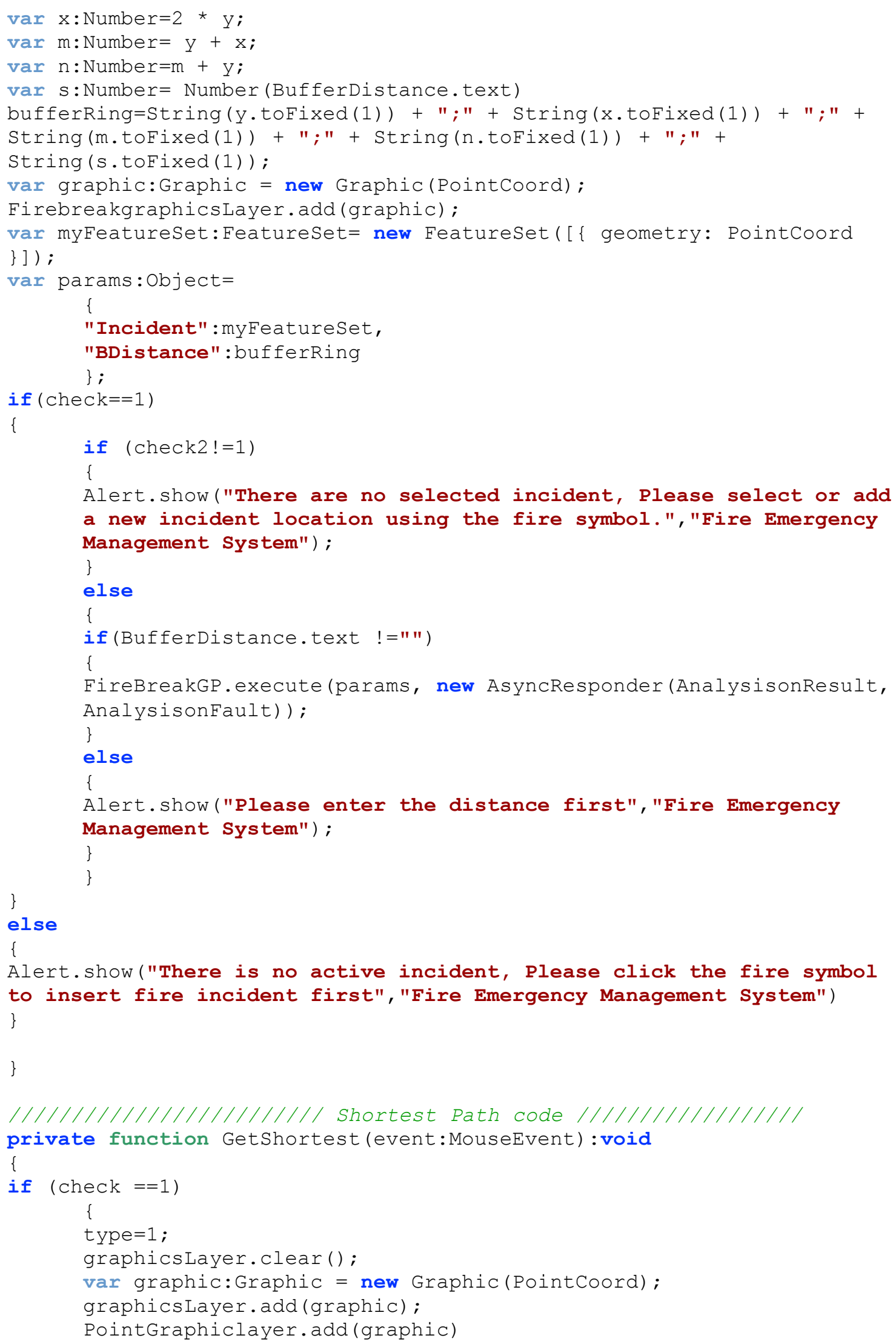




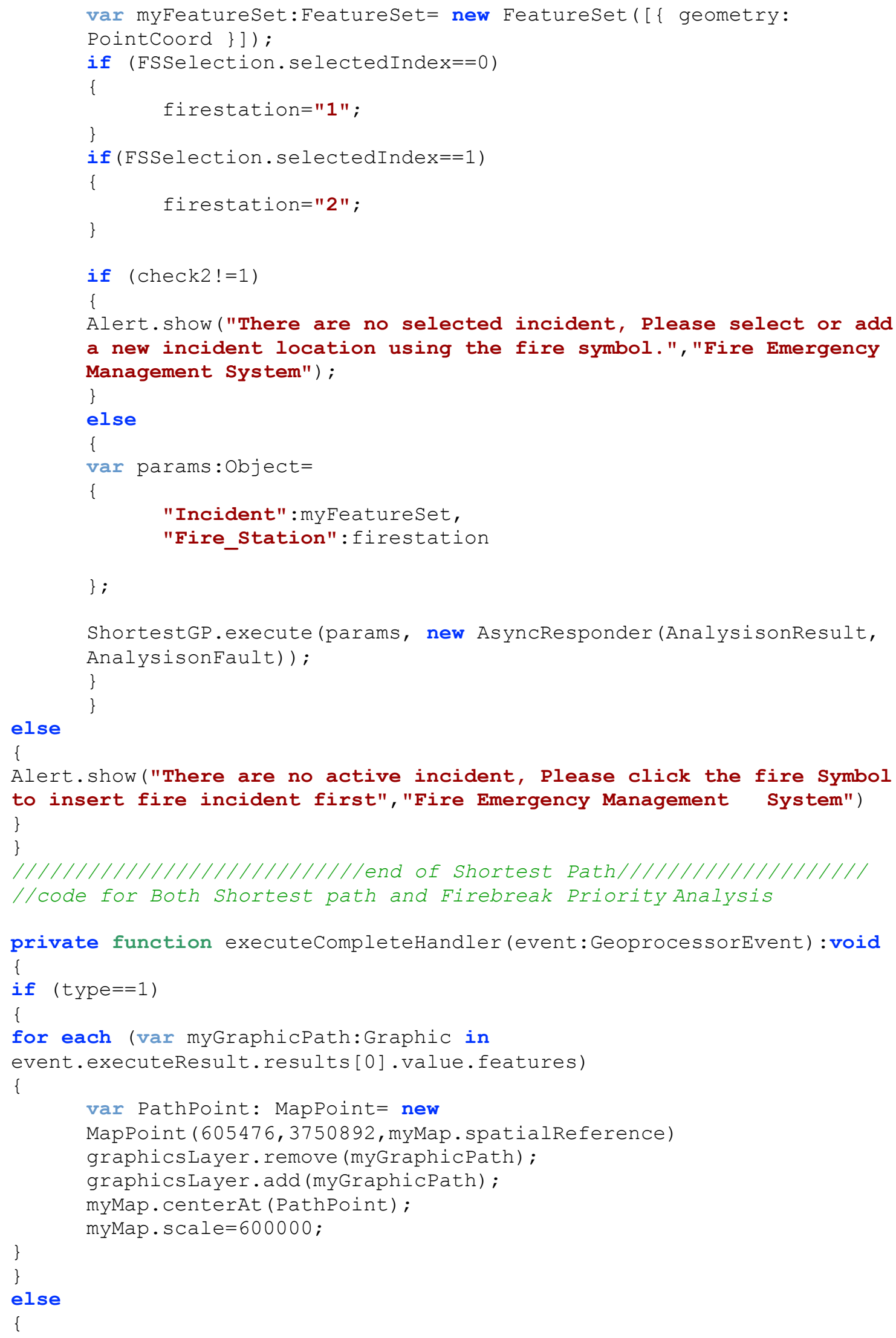




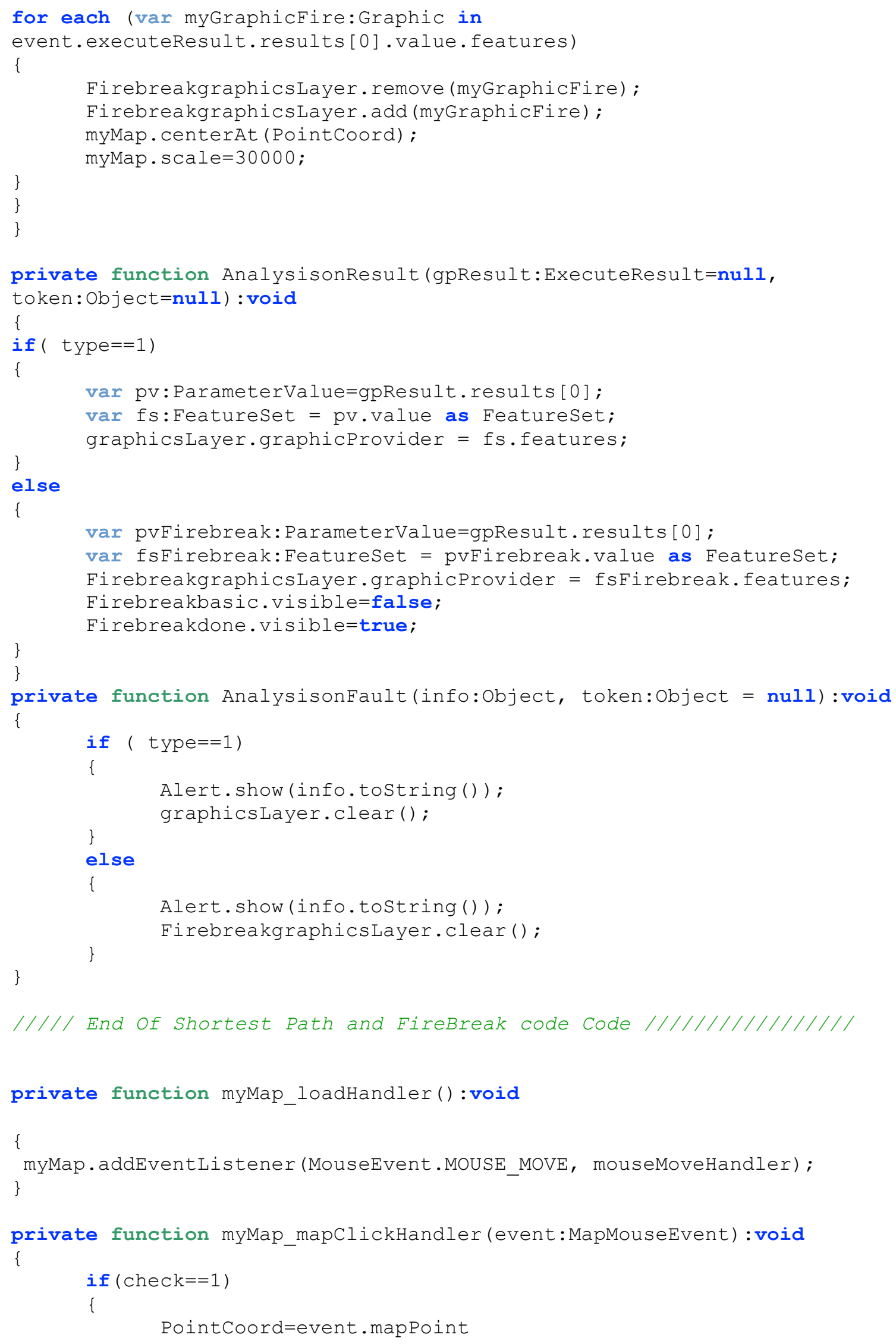




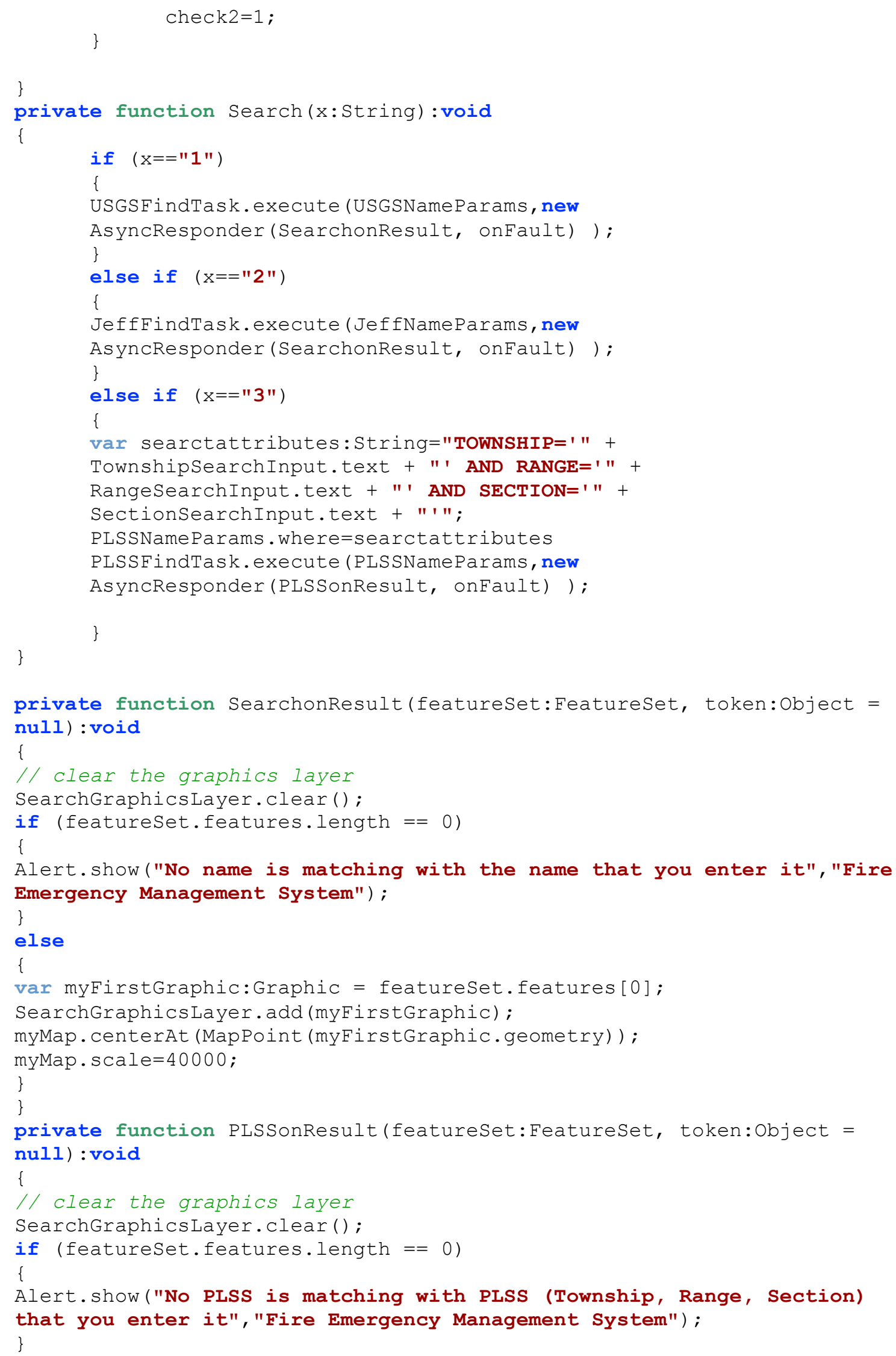




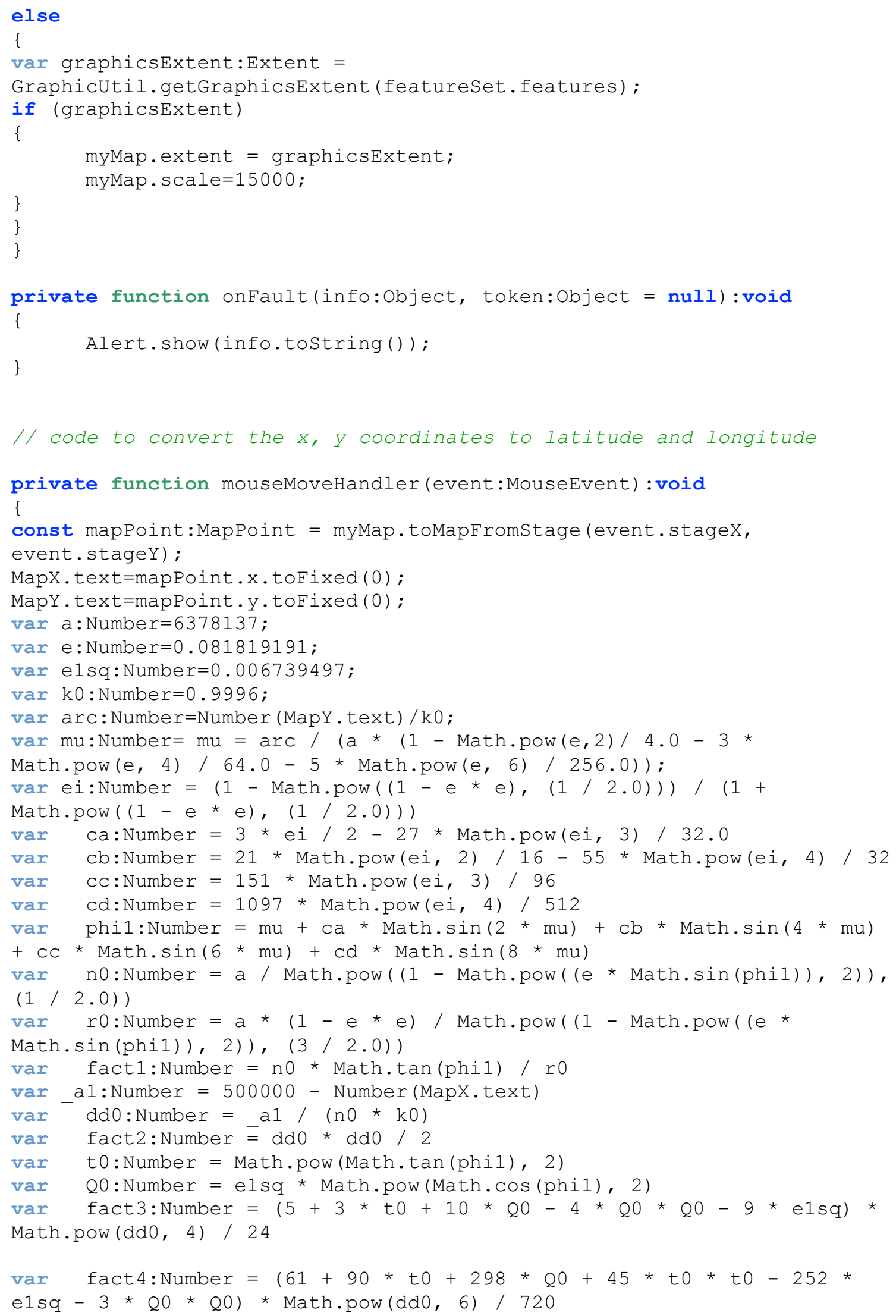




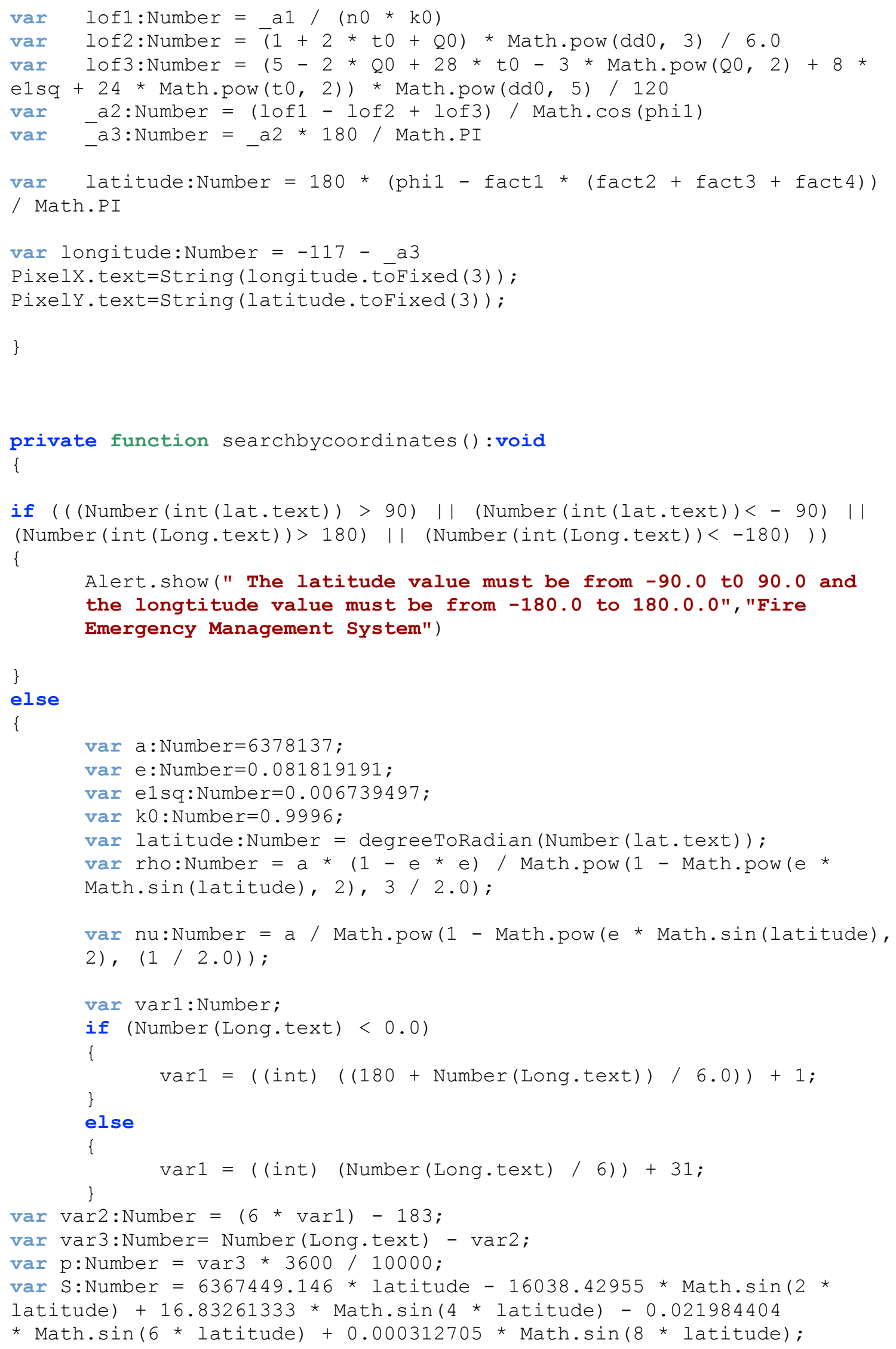




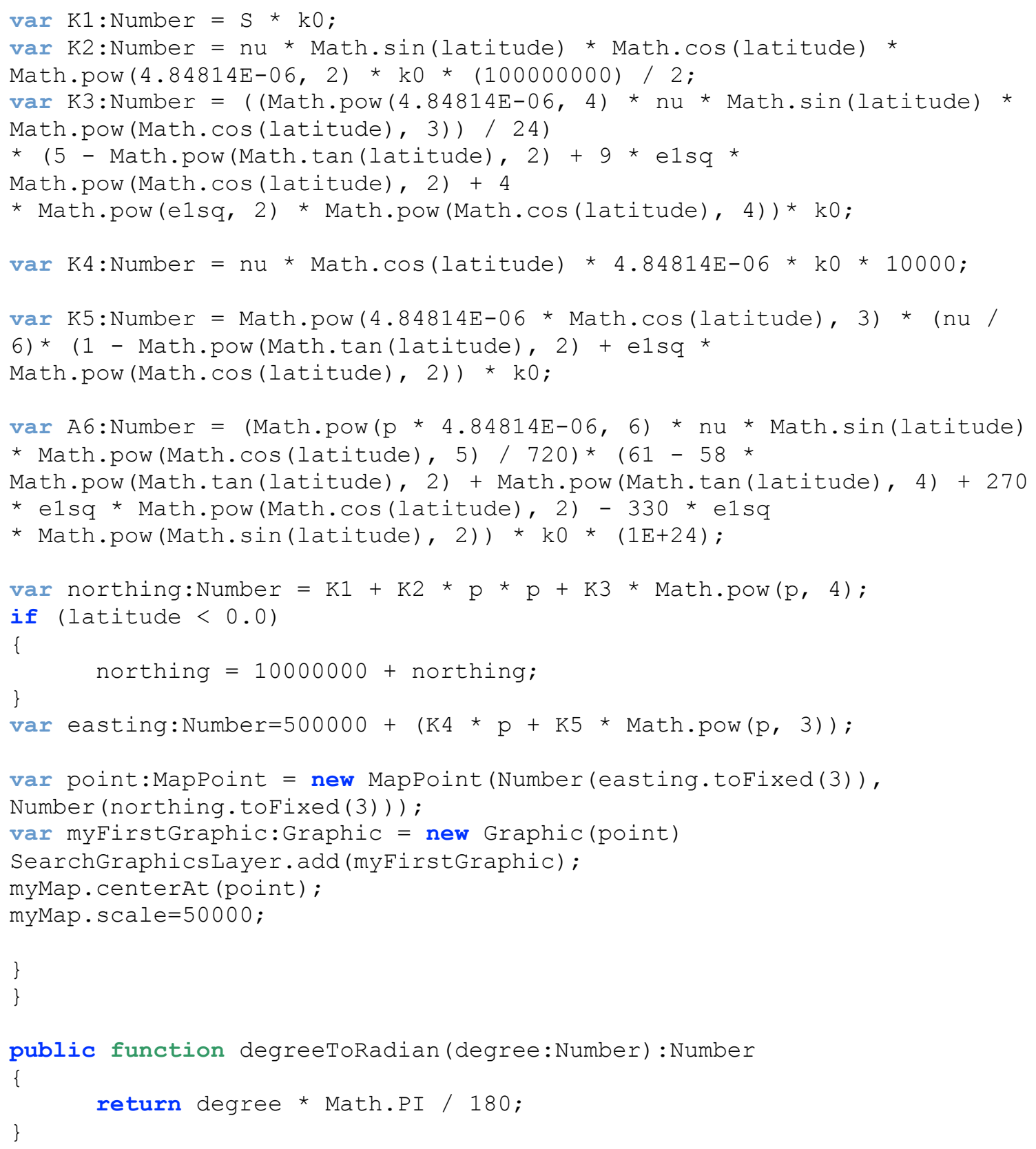




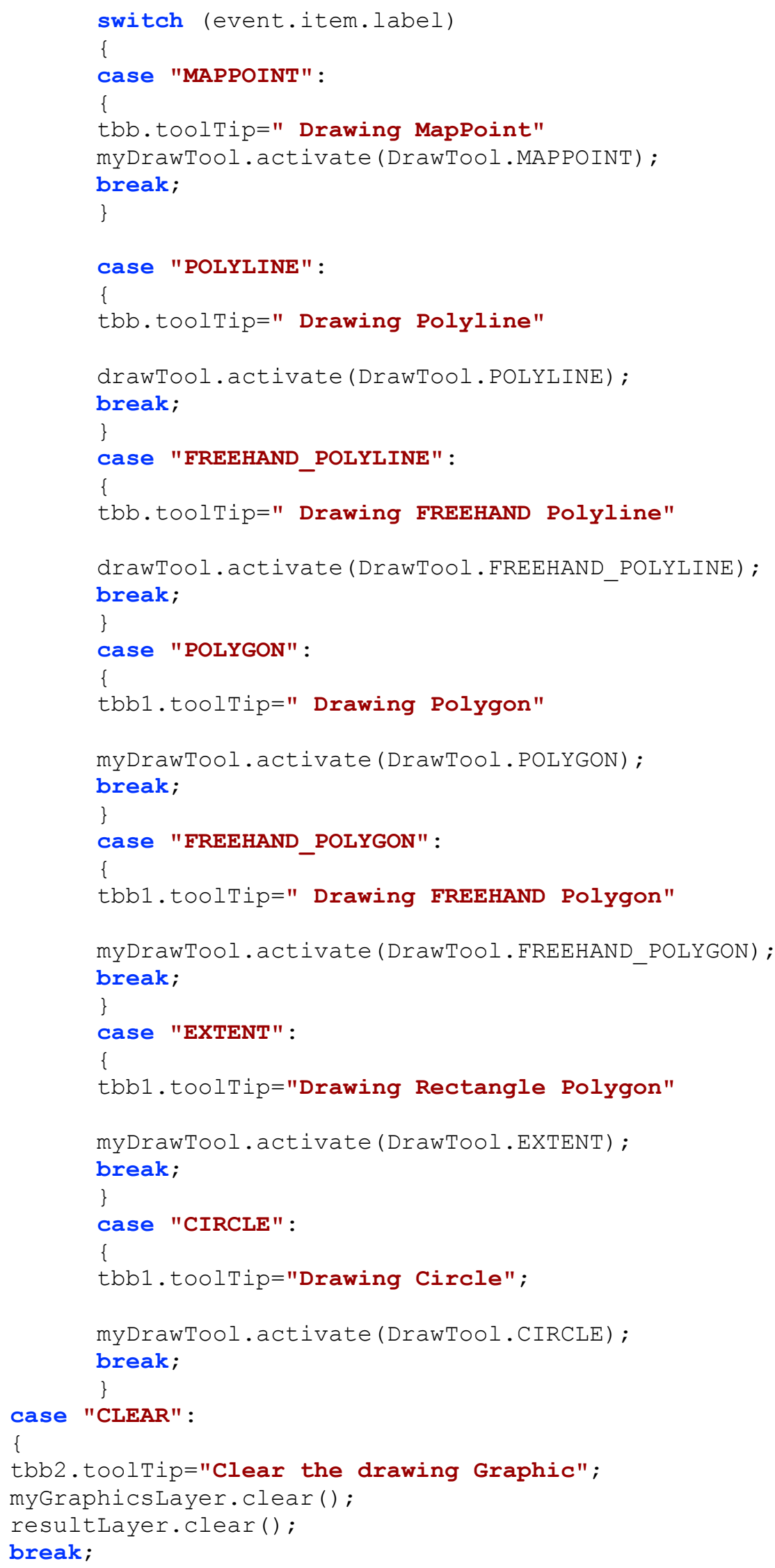




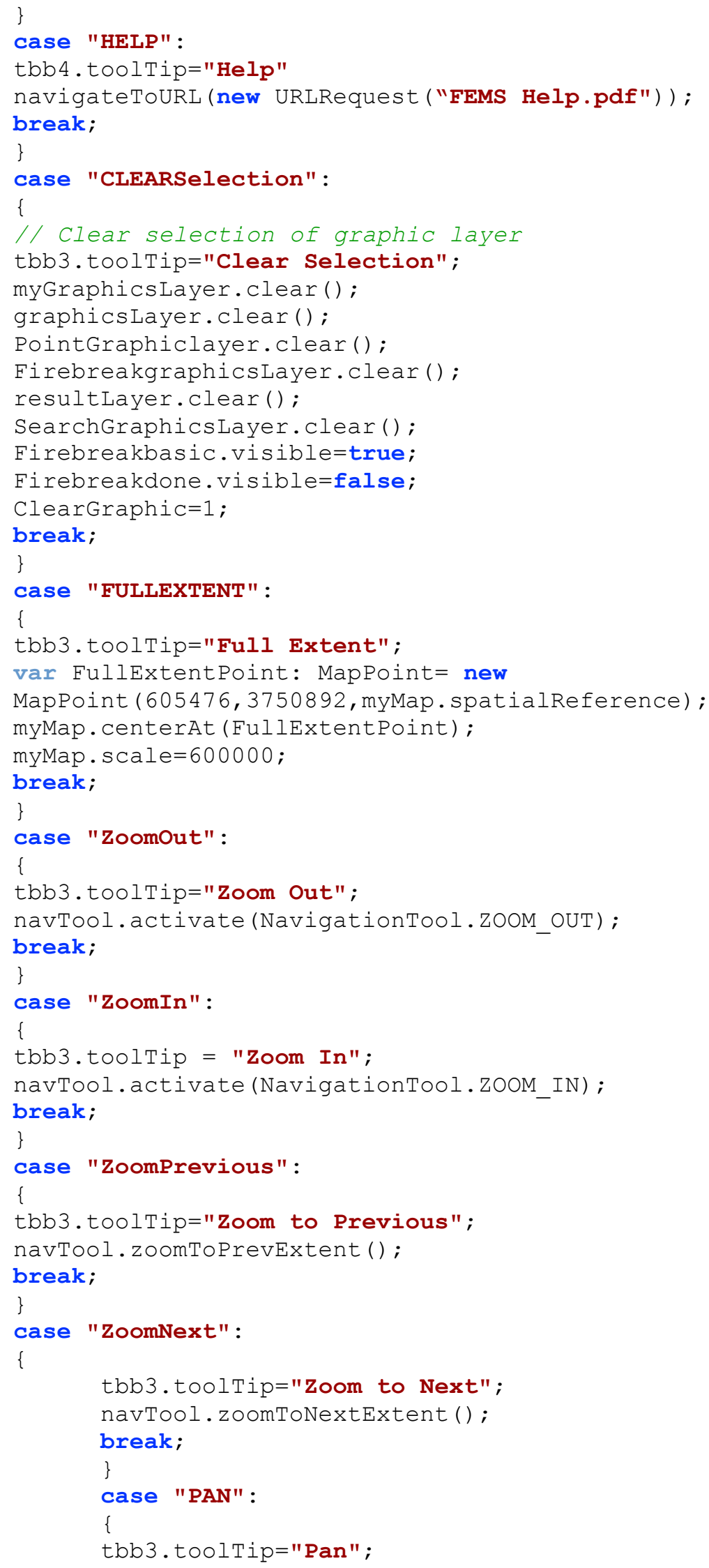




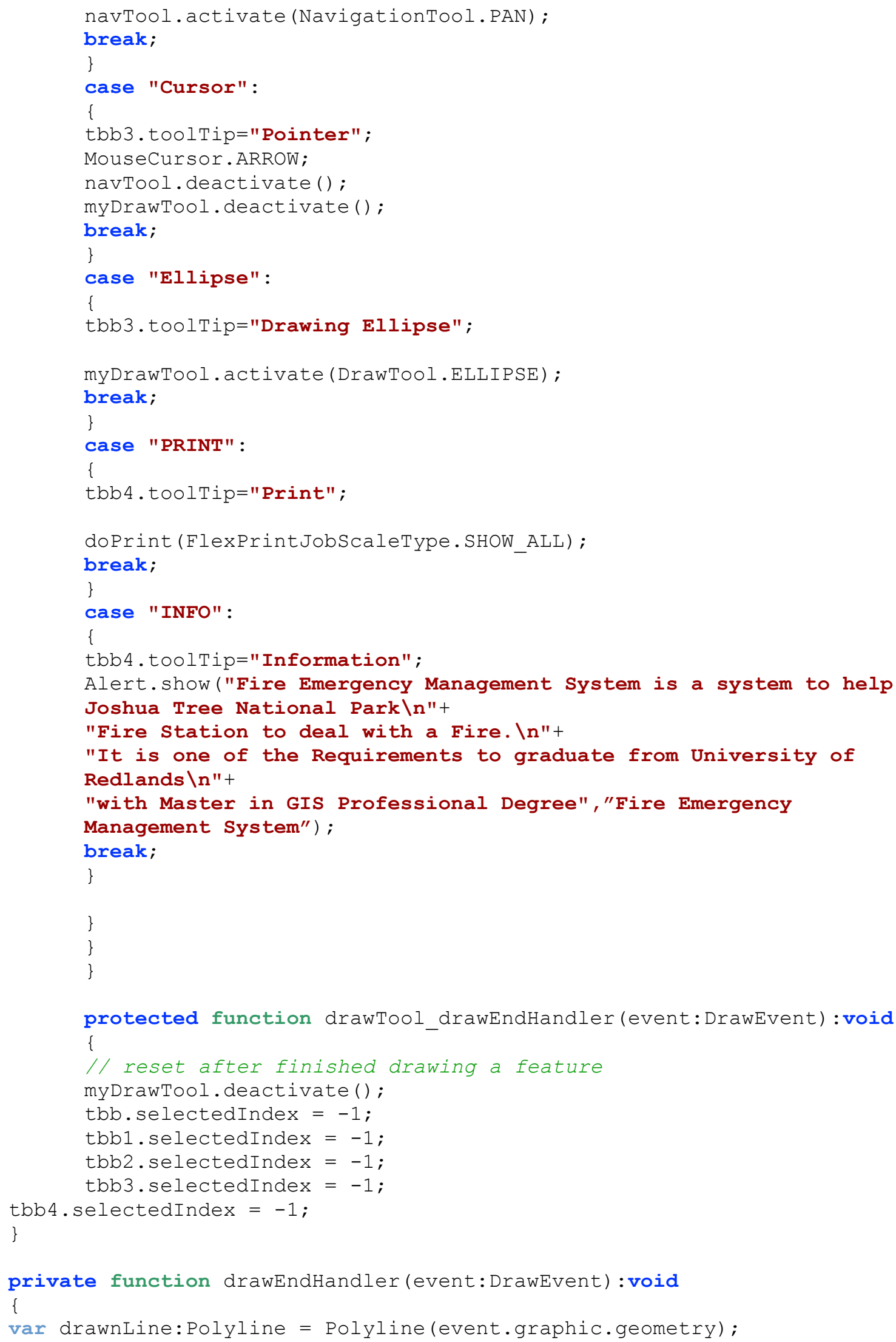




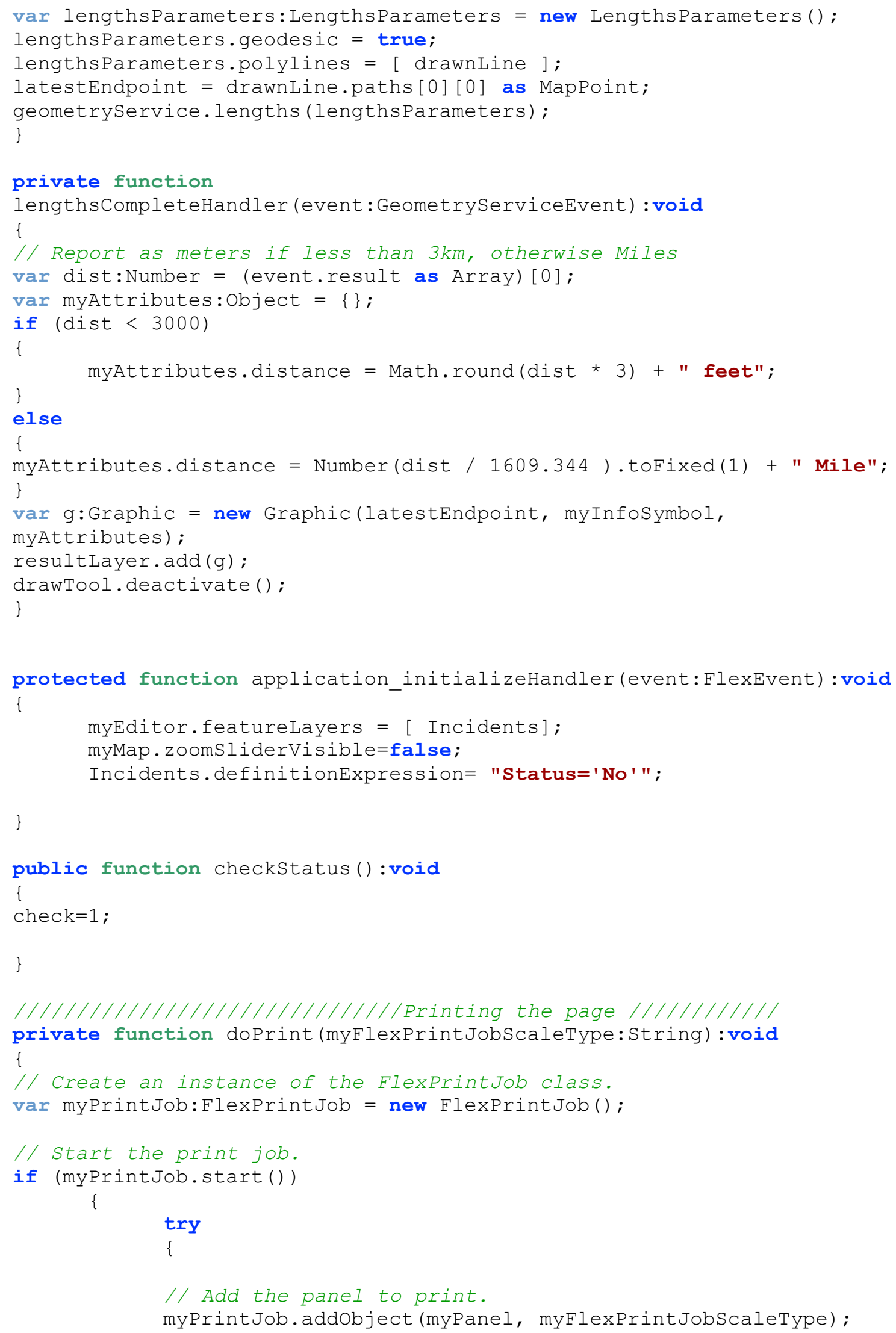




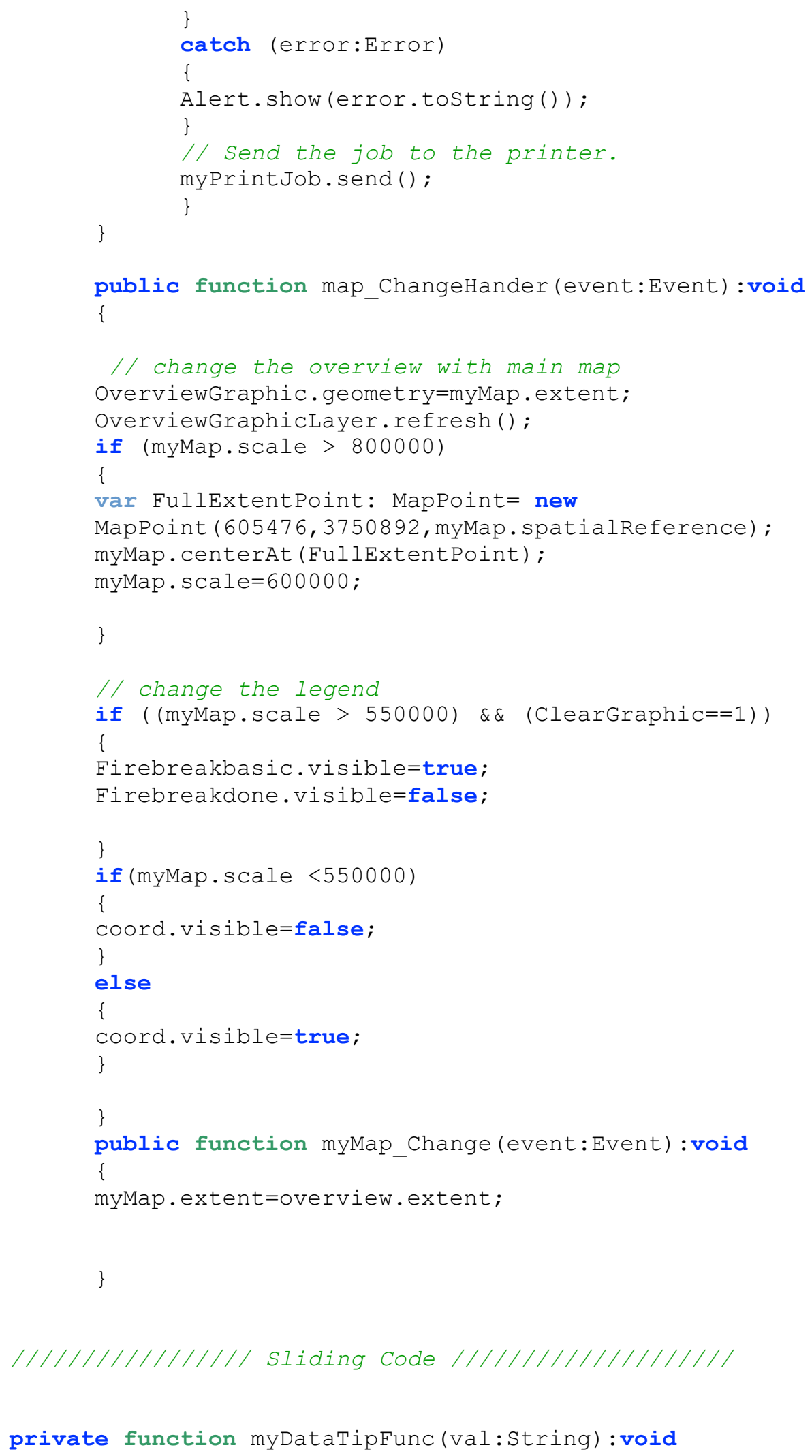


Main.alpha=1.0;

wild.alpha=0.6;

\}

else if (String (val) == "5")

\{

Main.alpha=0.6;

wild.alpha=1.0;

\}

else if (String (val) == "10")

\{

Main.alpha=0.0;

wild.alpha=1.0;

\}

\}

] ] $>$

$</$ fx:Script $>$

$<\mathrm{fx}$ :Declarations>

<!-- Navigation Tools Start -->

<Esri:NavigationTool id="navTool" map=" $\{$ myMap $\} " />$

$<\mathrm{mx}$ :TraceTarget includeCategory="true"

includeLevel="true"

includeTime="true"

level=" $\{$ LogEventLevel. DEBUG $\}$ " />

$<$ !-- Navigations Tool End -->

<Esi:Simplefillsymbol id="OverGraphicSymbol" alpha="0.0" >

<Esri:SimpleLinesymbol color="0x990000" width="4"/>

</Esri:Simplefillsymbol>

<!-- FireBreak Firebreak Priority Analysis Declarations-->

<Esri:SimplefillSymbol id="Inhabited" color="0x990000" alpha="1.0">

<Esri:SimpleLinesymbol color="0x000000" width="1"/>

</Esri:Simplefillsymbol>

<Esri:SimplefillSymbol id="Habitats" color="\#FFFF00" alpha="1.0"> <Esri:SimpleLineSymbol color="0x000000" width="1"/>

$</$ Esri:Simplefillsymbol>

<Esri:SimplefillSymbol id="Cultural" color="\#151B8D" alpha="1.0"> <Esri:SimpleLineSymbol color="0x000000" width="1"/>

$</$ Esri:Simplefillsymbol>

<Esri:Simplefillsymbol id="Vegetation" color="0x99FF33">

<Esri:SimpleLineSymbol color="0x000000" width="1"/>

$</$ Esri:Simplefillsymbol> 


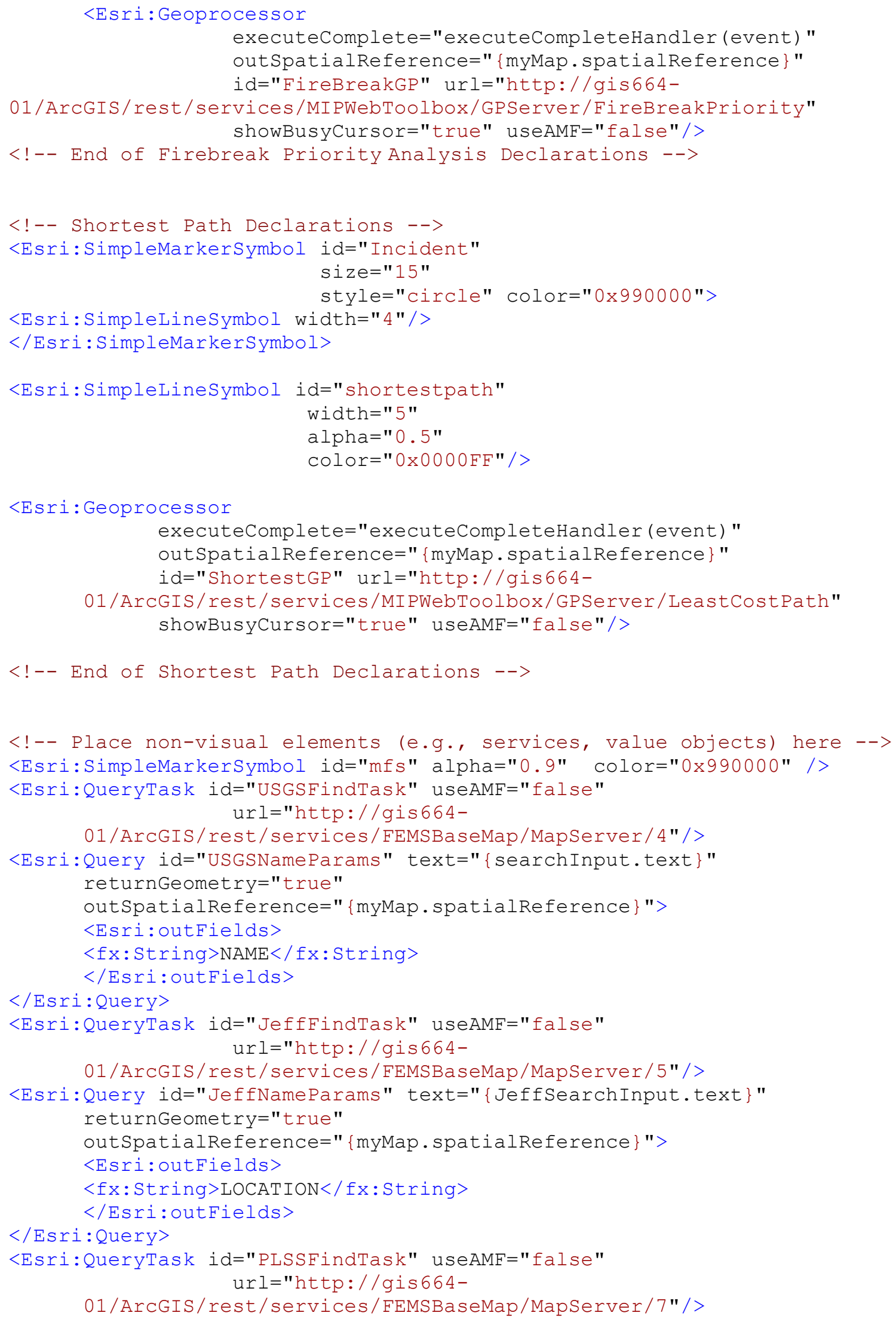




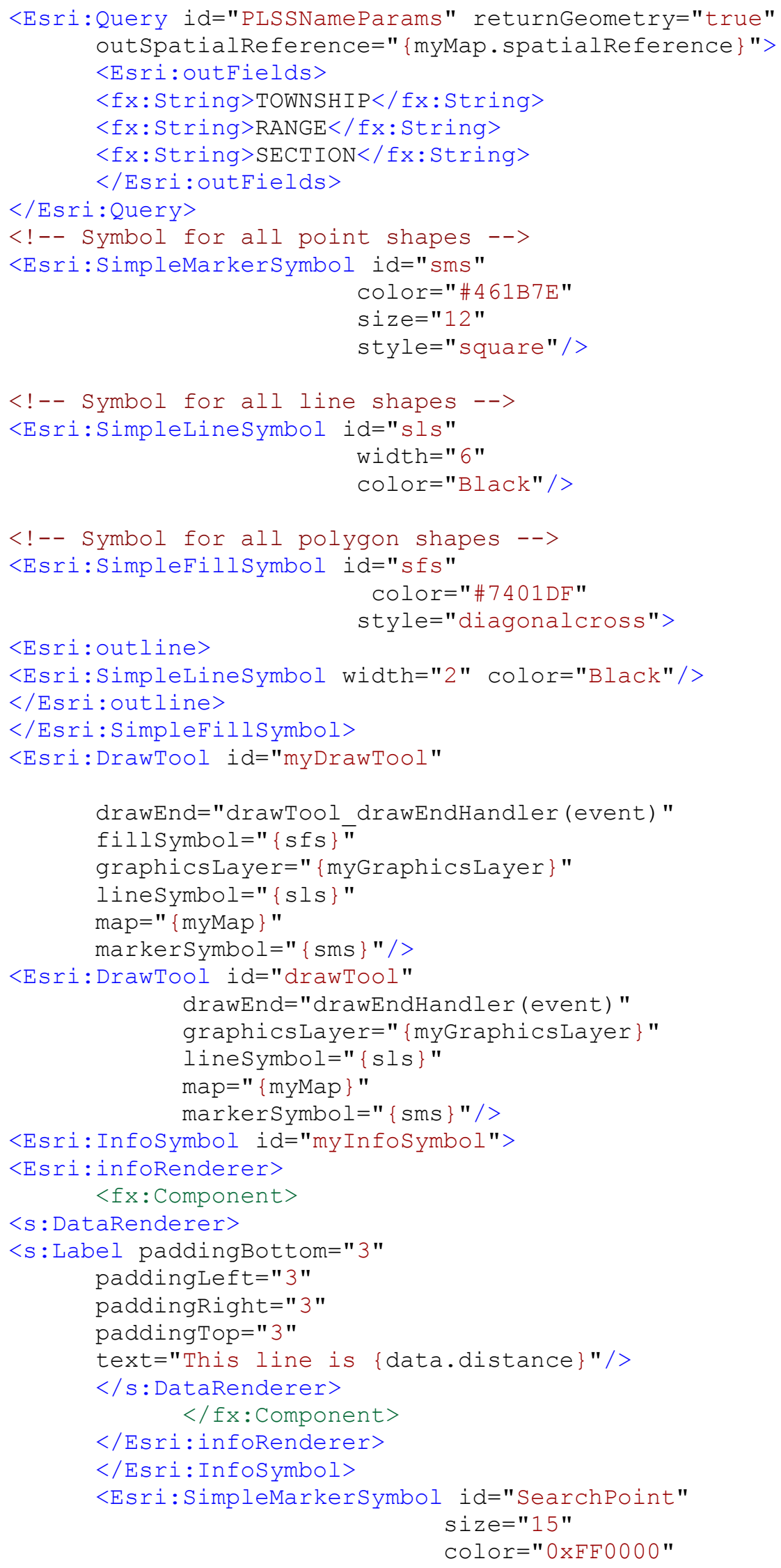




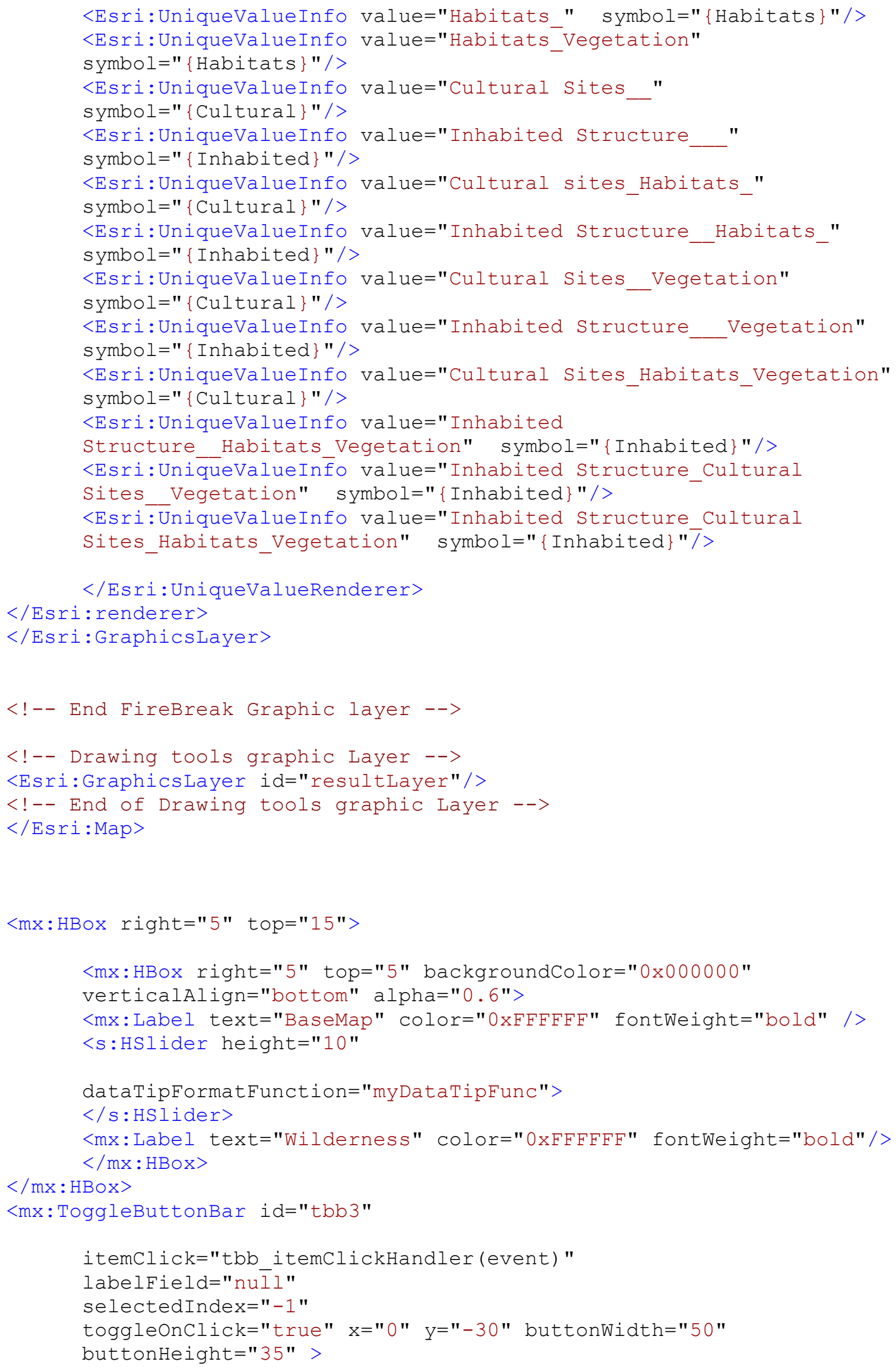


<fx:Object icon="@Embed (source='i clear.png')" label="CLEARSelection"/> <fx:Object icon="@Embed ( source='i_zoomfull.png')" label="FULLEXTENT"/> <fx:Object icon="eEmbed (source='i_zoomout.png')" label="Zoomout" id="zoomout"/>

<fx:Object icon="@Embed (source='i_zoomin.png')" label="ZoomIn" id="zoomin"/>

<fx:Object icon="@Embed ( source='i zoomprevious.png') "

label="ZoomPrevious"/>

<fx:Object icon="@Embed (source='i_zoomnext.png')" label="ZoomNext"/>

<fx:Object icon="@Embed ( source='i_pan.png')" label="PAN"/>

<fx:Object icon="@Embed (source='i_expand.png)" label="Cursor"/>

$</ m x$ : ToggleButtonBar $>$

<mx: ToggleButtonBar id="tbo4"

itemclick="tbb itemclickHandler(event)"

labelField="nūil"

selectedIndex="-1"

toggleonclick="true" $\mathrm{x}=$ "780" $\mathrm{y}=$ "-30" buttonWidth="50"

height="35">

<fx:Object icon="@Embed (source='i_help.png')" label="HELP" id="help"/>

<fx:Object icon="@Embed (source='i about.png')" label="INFO"/>

<fx:Object icon="@Embed (source='i_print.png')" label="PRINT"/>

$</ m x$ : ToggleButtonBar>

$<\operatorname{mx}:$ HBox width="100\%" bottom="10" $\mathrm{x}=" 215$ " id="coord">

$<$ s:Label $x=" 215 "$ id="maplb" text="Map X Easting:" color="\#000000"

bottom="10" fontWeight="bold" fontSize="16"/>

<s:Label $\mathrm{x}=$ "270" bottom="10" id="MapX" color="white" fontWeight="bold"

fontSize="16"/>

<s:Label $\mathrm{x}=$ "330" bottom="10" text="Map y Northing:" color="\#000000"

fontWeight="bold" fontSize="16"/>

<s:Label $\mathrm{x}=$ "380" bottom="10" id="MapY" color="white" fontWeight="bold"

fontsize="16"/>

$<$ s:Label $x=" 450 "$ bottom="10" text="Latitude:" color="\#000000"

fontWeight="bold" fontSize="16"/>

<s:Label $\mathrm{x}=$ "520" bottom="10" id="Pixely" color="white"

fontWeight="bold" fontSize="16"/>

$<$ :Label $\mathrm{x}=$ "620" bottom="10" text="Longitude:" color="\#000000"

fontWeight="bold" fontSize="16"/>

$<$ s:Label $x=" 705 "$ bottom="10" id="Pixelx" color="white"

fontWeight="bold" fontSize="16"/>

$</ \operatorname{mx}:$ HBox $>$

$</ \mathrm{s}:$ Panel $>$

$</ \mathrm{s}$ : BorderContainer $>$

$<$ : BorderContainer $\mathrm{x}=$ "0" $\mathrm{y}=$ "1" width="100\%" height="82"

borderColor="\#F87431" backgroundColor="Black" cornerRadius="10">

$<$ s:backgroundFili>

$<$ s:LinearGradient rotation="90">

$<$ : GradientEntry color="0xE2E2E2" />

$<$ s:GradientEntry color="0x323232" />

$</ \mathrm{s}$ : LinearGradient $>$

$</$ s:backgroundFill> 


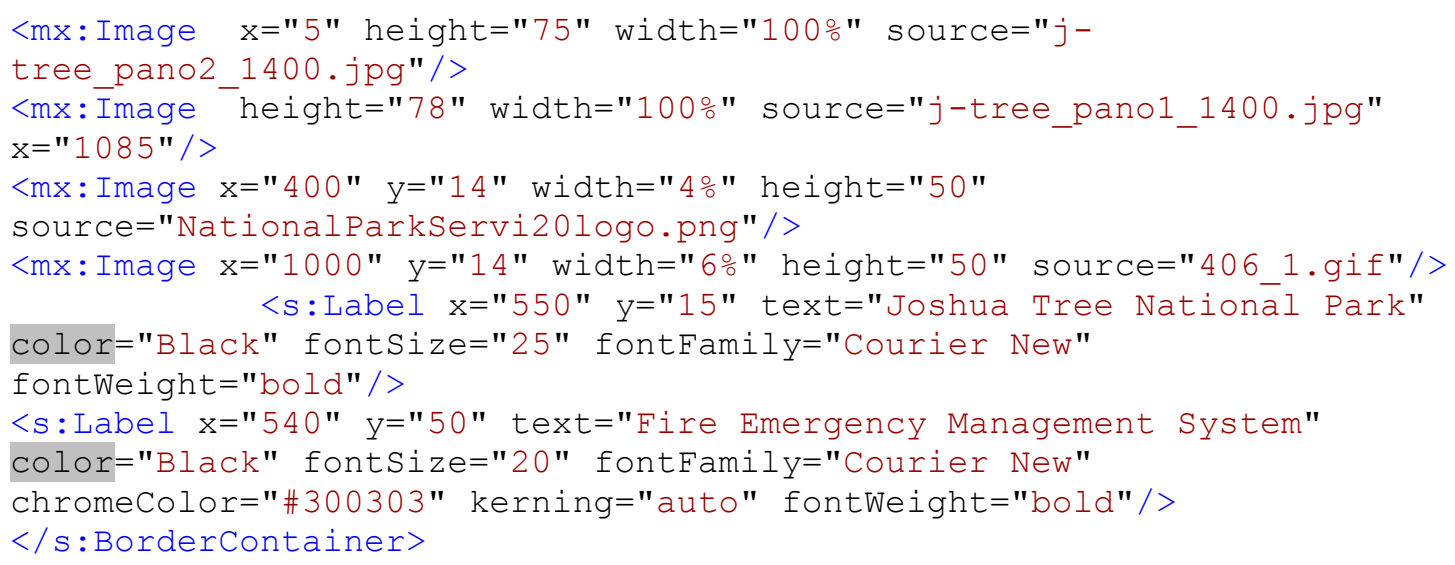


\&amp; \&amp; (RangeSearchInput.text!=' ') \&amp; \&amp;

(SectionSearchInput.text!='') \}" click="Search('3') "/>

$</$ s: NavigatorContent $>$

<s:NavigatorContent label="Search By Coordinates" width="90\%"

height="100\%" backgroundColor="\#736F6E" $\mathrm{x}=$ "20">

$<\mathrm{s}$ :Label $\mathrm{y}=" 10 " \mathrm{x}=" 10 "$ text="Example: -90.0 to + 90.0" color="White"/>

$<$ s:Label $y=" 30 " \mathrm{x}=" 10 "$ text="Latitude" color="Black"/>

$<$ s:TextInput id="lat" $y=" 50 " \mathrm{x}=$ "40"/>

$<$ : Label y="80" $\mathrm{x}=" 10 "$ text="Example: -180.0 to + 180.0" color="White"

$1>$

$<$ s:Label $y=" 100 " x=" 10 "$ text="Longitude" color="Black"/>

$<$ s:TextInput id="Long" $y=" 115 " x=" 40 " \quad />$

$<$ s:Button $\mathrm{x}=$ "60" y="150" label="Search" enabled=" $\{$ Long.text != ' ' $\}$ "

click="searchbycoordinates ()" />

$</ s$ :NavigatorContent $>$

$</ \mathrm{mx}$ : Accordion $>$

$</ s$ :BorderContainer $>$

$<\mathrm{s}$ :BorderContainer top="390" $\mathrm{x}=" 12$ " width="16\%" height="100\%"

borderColor="\#F87431" cornerRadius="10">

$<$ : backgroundFill $>$

$<$ s:LinearGradient rotation="90">

$<$ s:GradientEntry color="0xE2E2E2" />

$<$ s:GradientEntry color="0x323232" />

$</ \mathrm{s}:$ LinearGradient>

$</ \mathrm{s}$ : backgroundFill>

$<$ s:Label $y=" 10 " x=" 40 "$ text="Selected Fire Station" color="Black"/> $<$ mx:ComboBox id="FSSelection" selectedIndex="0" horizontalcenter="0" $\mathrm{x}=" 10 " \mathrm{y}=" 30 "$ width="70\%" >

$<\mathrm{fx}:$ Array $>$

<fx:String $>$ NPS Black Rock FireStation</fx:String $>$

$<f x$ :String $>$ BLM Firestation</fx:String $>$

$</$ fx:Array $>$

$</$ mx: ComboBox $>$

$<$ :Panel $\mathrm{x}=$ "20" $\mathrm{y}=$ "90" title="Shortest Path to Incident"

alignmentBaseline="ideographicCenter" width="80\%" height="65"

cornerRadius="10" >

$<$ s:Button id="LeastCostPath" label="Shortest Path" $\mathrm{x}=$ "35" y="5"

click="GetShortest (event)" />

$</ \mathrm{s}:$ Panel $>$

<s:Panel $\mathrm{x}=$ "13" $\mathrm{y}=$ "170" title="Firebreak Priority Analysis"

alignmentBaseline="ideographicCenter" width="85\%" height="120"

cornerRadius="10" >

$<$ : Label text="Enter the Distance (Miles)" $\mathrm{x}=$ "10" $y=" 5 " />$

$<$ s:TextInput $\mathrm{x}=$ "35" $\mathrm{y}=$ "20" id="BufferDistance"/>

$<$ :Button id="FireBreak" label="Firebreak" x="55" y="50"

click="GetFireBreak (event)" />

$</ \mathrm{s}:$ Panel $>$

$</ \mathrm{s}$ :BorderContainer $>$

<s:BorderContainer $\mathrm{x}=" 1198 " \mathrm{y}=$ "90" width="16\%" height="180"

borderColor="\#F87431" cornerRadius="10">

<s:backgroundFill>

<s:LinearGradient rotation="90"> 


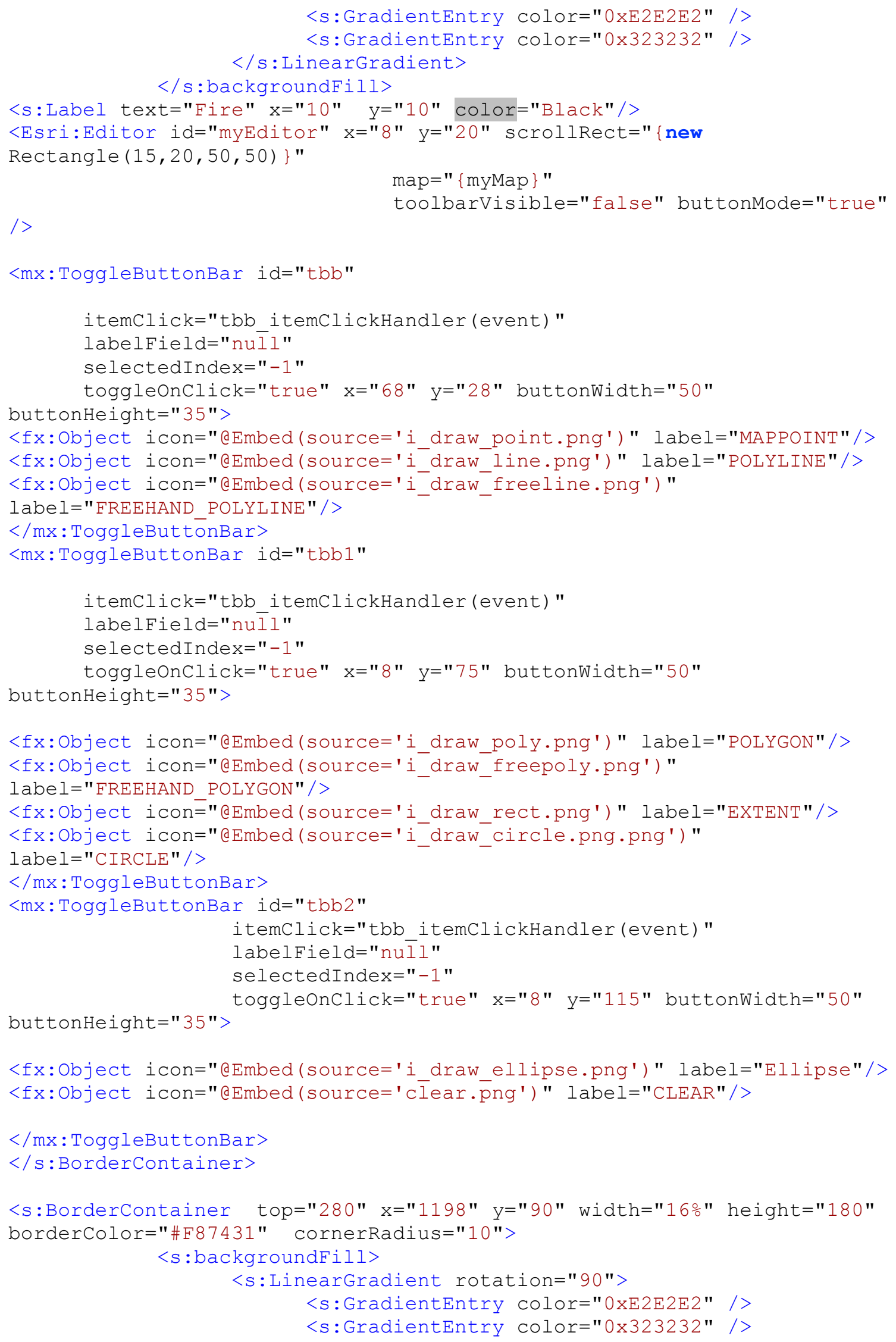




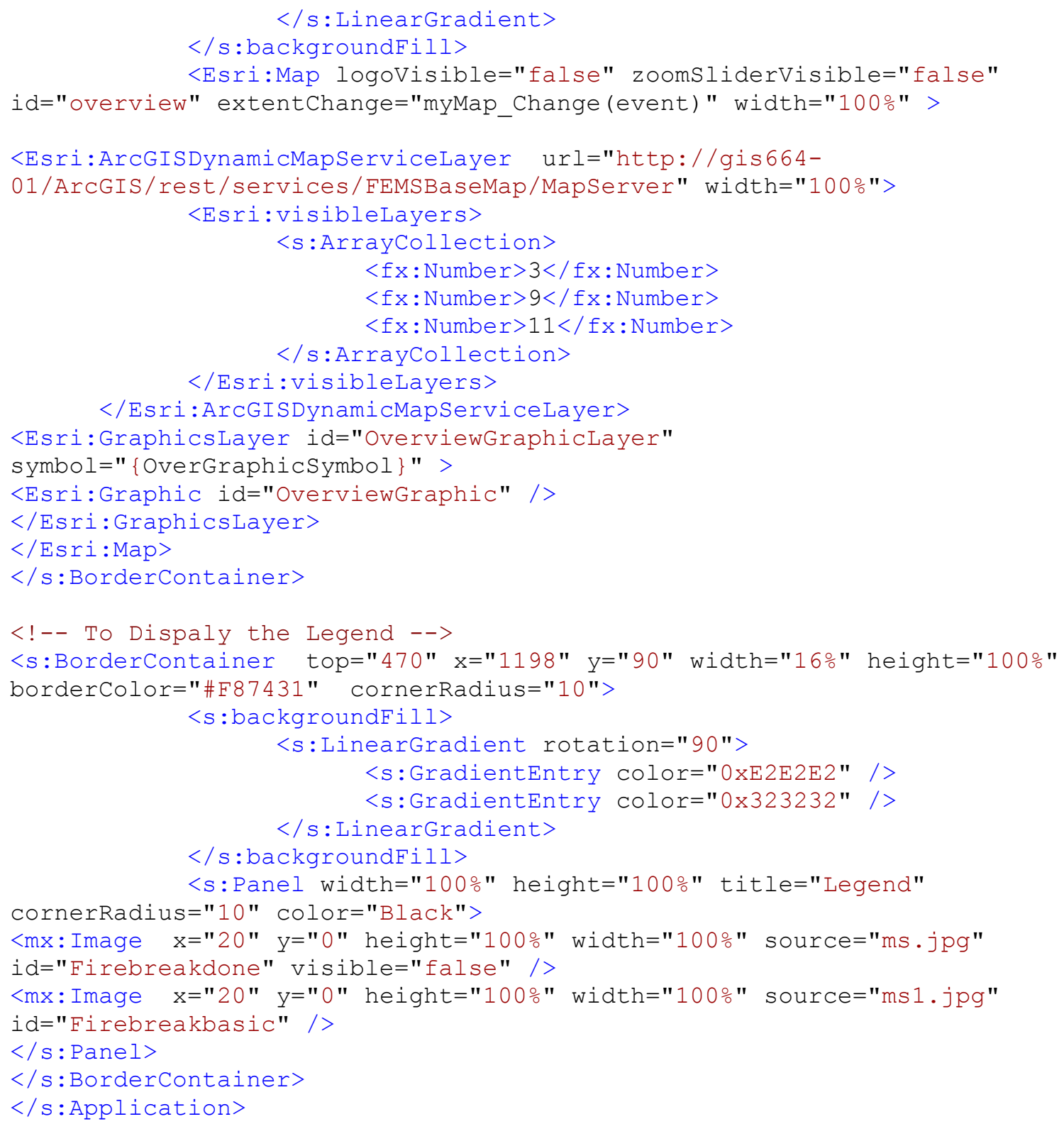

